\title{
High-order discontinuous Galerkin methods on polyhedral grids for geophysical applications: seismic wave propagation and fractured reservoir simulations*
}

\author{
Paola F. Antonietti, Chiara Facciolà, Paul Houston, Ilario Mazzieri, \\ Giorgio Pennesi, Marco Verani
}

\begin{abstract}
We present a comprehensive review of the current development of PolyDG methods for geophysical applications, addressing as paradigmatic applications the numerical modeling of seismic wave propagation and fracture reservoir simulations. We first recall the theoretical background of the analysis of PolyDG methods and discuss the issue of its efficient implementation on polytopic meshes. We address in detail the issue of numerical quadrature and recall the new quadrature free algorithm for the numerical evaluation of the integrals required to assemble the mass and stiffness matrices introduced in [22]. Then we present PolyDG methods for the approximate solution of the elastodynamics equations on computational meshes consisting of polytopic elements. We review the well-posedness of the numerical formulation and $h p$-version a priori stability and error estimates for the semi-discrete scheme, following [10]. The computational performance of the fully-discrete approximation obtained based on employing the PolyDG method for the space discretization coupled with the leap-frog time marching scheme are demonstrated through numerical experiments. Next, we address the problem of modeling the flow in a fractured porous medium and we review the unified construction and analysis of PolyDG methods following [16]. We show, in a unified setting, the well-posedness of the numerical formulations and $h p$-version a priori error bounds, that are then validated through numerical tests. We also briefly discuss the extendability of our approach to handle networks of partially immersed fractures and networks of intersecting fractures, recently proposed in [15].
\end{abstract}

Paola F. Antonietti

MOX-Laboratory for Modeling and Scientific Computing, Dipartimento di Matematica, Politecnico di Milano, Piazza Leonardo da Vinci 32, 20133 Milano, e-mail: paola.antonietti@polimi.it

Chiara Facciolà

MOX-Laboratory for Modeling and Scientific Computing, Dipartimento di Matematica, Politecnico di Milano, Piazza Leonardo da Vinci 32, 20133 Milano, e-mail: chiara.facciola@polimi.it

Paul Houston

School of Mathematical Sciences, The University of Nottingham, University Park, Nottingham, NG7 2RD, UK, e-mail: paul . hou st on 0 nottingham.ac.uk

Ilario Mazzieri

MOX-Laboratory for Modeling and Scientific Computing, Dipartimento di Matematica, Politecnico di Milano, Piazza Leonardo da Vinci 32, 20133 Milano, e-mail: ilario.mazzieriepolimi.it

Giorgio Pennesi

MOX-Laboratory for Modeling and Scientific Computing, Dipartimento di Matematica, Politecnico di Milano, Piazza Leonardo da Vinci 32, 20133 Milano, e-mail: giorgio.pennesi@polimi.it

Marco Verani

MOX-Laboratory for Modeling and Scientific Computing, Dipartimento di Matematica, Politecnico di Milano, Piazza Leonardo da Vinci 32, 20133 Milano, e-mail: marco.veraniepolimi.it

* P.F.A. and M.V. acknowledge the financial support of MIUR thourgh the PRIN grant n. 201744KLJL. P.F.A., M.V. and I.M have also been funded by INdAM-GNCS. 


\section{Introduction}

Many geophysical and engineering applications, including, for example, fluid-structure interaction, crack and wave propagation phenomena, and flow in fractured porous media, are characterized by a strong complexity of the physical domain, possibly involving faults and/or fractures, heterogeneous media, moving geometries/interfaces and complex topographies. Whenever classical finite element methods are employed to discretize the underlying differential model, the process of mesh generation can represent a severe bottleneck for the simulation process, as classical finite element methods (in three-dimensions) typically only support computational grids composed of tetrahedral/hexahedral/prismatic/pyramidal elements. To overcome this limitation, in the last decade a wide strand of literature has focused on the design of numerical methods that support computational meshes composed of general polygonal and polyhedral (polytopic, for short) elements. In the conforming setting, we mention, for example, the Composite Finite Element Method, see, e.g., [104, 103], the Mimetic Finite Difference (MFD) method, see, e.g., [106, 60, 58, 59, 42, 7, 18], the Polygonal Finite Element Method, see, e.g., [140], the eXtended Finite Element Method, see, e.g., [88, 141, 97], and, more recently, the Virtual Element Method (VEM), see, e.g., [39, 46, 8, 48, 9, 47, 41, 40, 43, 45, 44]. In the setting of non-conforming/discontinuous polygonal methods, we mention, for example, Composite Discontinuous Galerkin Finite Element methods [19, 20], Hybridizable Discontinuous Galerkin methods [75, 76, 77, 78], the Hybrid High-Order (HHO) method [86, 72, 85, 84, 1, 55, 53, 71, 87, 54], the non-conforming VEM [24, 35, 68], and Gradient Schemes [90]. This article focuses on discontinuous Galerkin methods on polytopic grids (PolyDG), which represent the natural extension of the classical discontinuous Galerkin method on tetrahedral/hexahedral grids to meshes composed of arbitrarily-shaped polytopic elements. Due to the fact that the discrete space is constructed based on employing piecewise discontinuous polynomials, DG methods are naturally suited to robustly support polytopic meshes. In fact, in the last few years intensive research has been undertaken on this topic; in particular, we refer here to the pioneering works $[12,37,38,36,67]$, the more recent results $[63,21,26,22,30,5,11]$, and refer to $[13,66]$, and the reference therein, for a comprehensive review.

This article focuses on two challenging applications in geophysics, namely, seismic wave propagation and fractured reservoir simulations and presents a review of PolyDG methods for this class of problems, as well a detailed discussion on the development of efficient quadrature rules on polytopic elements that allows a massively-parallel implementation of PolyDG methods on parallel architectures. From the mathematical and modeling viewpoints, these two paradigmatic applications share a number of challenges. For example, they both require, at the same time $i$ ) a flexible description of the domain involving multiple scales, interfaces, network of fractures, and strongly heterogeneous media; and ii) an accurate representation of the solution field, particularly for wave propagation phenomena, where a sufficiently high number of nodes per wavelength is needed to keep numerical dispersion and dissipation errors low. PolyDG methods are perfectly suited to tame all these mathematical and numerical challenges, indeed $i$ ) they are naturally oriented towards high-order approximations, in any space dimension, and feature a high-level of intrinsic parallelism; ii) the dimension of the local approximation space only depends on the local approximation order, and is independent of the shape of an element and the number of faces/edges of an element. As a consequence, in contrast to other polytopic finite element methods, on agglomeration based meshes the dimension of the local space remains under control; iii) they can handle mesh elements with possibly an unbounded number of faces and face/edge degeneration can be supported. We point out that the last feature is very important in practical applications, since it allows for hybrid mesh algorithms that efficiently deal with heterogeneous media, localized geological/topographic irregularities, faults and fractures characterizing geophysical applications. The main idea consists in generating an initial (hexahedral/tetrahedral in three dimensions, for example) mesh, based on employing standard mesh generators; then elements intersecting the geological irregularities are suitably cut and/or agglomerated, thus generating polytopes, while keeping a regular structure elsewhere, cf. Figure 1 for an illustrative example. Beyond the simplicity of generating the computational hybrid grids based on a convenient combination of hexahedral/tetrahedral/polyhedral elements, one of the other advantages of polytopic decompositions over standard simplicial/hexahedral grids is that, even on relatively simple geometries, the average number of elements needed to discretize complicated domains is substantially smaller $[19,20]$, without enforcing any domain approximation. This advantage becomes even more evident whenever the domain contains complex geometrical features (large number of fractures, fractures intersecting with small angles, etc.) and the underlying grid is chosen to be matching with the interfaces.

In the following we provide a brief description of the contents of each of the following sections, and highlight their scientific importance within the community. In Section 2 we introduce the notation and the key theoretical results 


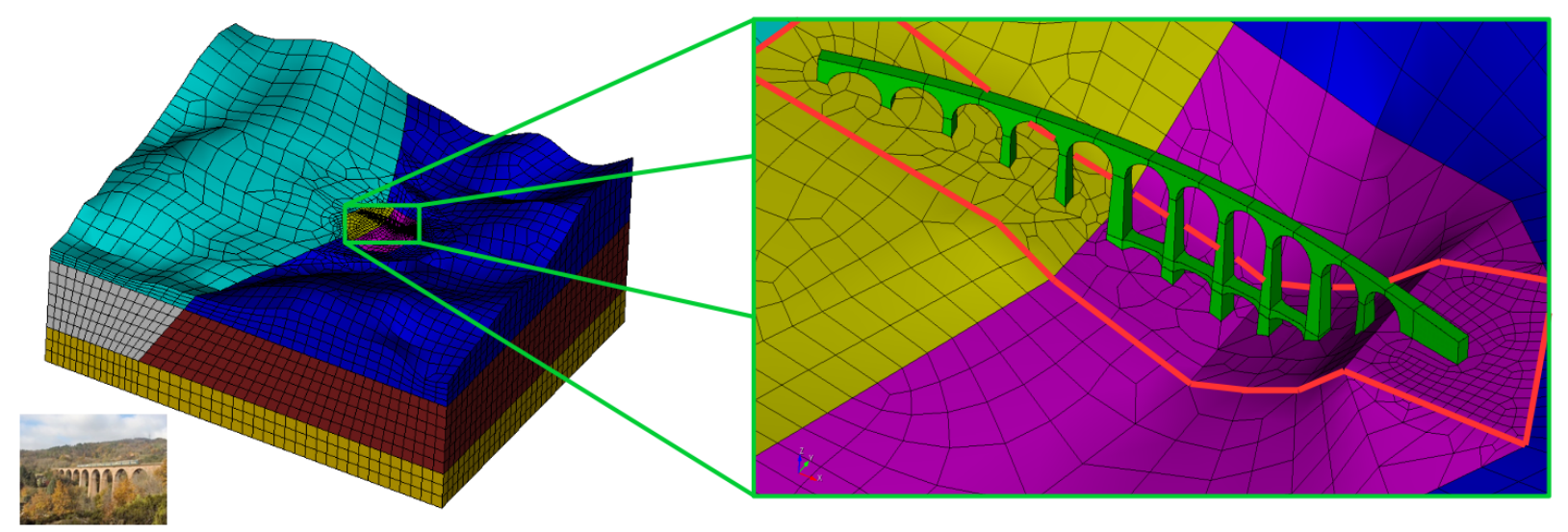

Fig. 1 A three-dimensional example of hybrid hexahedral/polyhedral grid of the Acquasanta railway bridge on the Genoa-Ovada railway (Italy). The mesh is obtained by exploiting the flexibility of polyhedral elements near the Acquasanta bridge (cf. the region delimitated by the red line of the zoom) while keeping a regular structure elsewhere

needed to analyze PolyDG approximations. In particular, we summarize the main theoretical results concerning this class of methods outlined in $[67,13,63,66]$. Following $[63,66]$, we start from the generalization of the standard shape-regularity property to polytopic elements and introduce some trace and inverse inequalities and polynomial approximation properties of the underlying discrete spaces. These results represent the main tools for handling elements with a degenerating and/or unlimited number of faces/edges. The contents of this section form the basis for the theoretical analysis of the discretization schemes for seismic wave propagation and flow in fractured porous media presented in the second part of the manuscript.

Section 3 focuses on the construction and outline of a new technique for the efficient computation of integrals of polynomial functions over convex and non-convex polytopic domains, and its application to the numerical computation of the terms appearing in the weak formulation of PolyDG methods. The classical (and most widely employed) approach for the integration of polynomial functions over polytopes is the so called sub-tessellation method: here, the domain of integration is sub-divided (sub-tessellated) into $d$-simplices, whereby standard quadrature rules are employed, cf. $[137,117,150,122,139,101]$ and also $[115,151]$ for a similar quadrature approach where the polytopic domain of integration is sub-tessellated into $d$-parallelograms. However, the sub-tessellation method is generally computationally expensive as it leads to a very large number of function evaluations, particularly when the integrand is a high order polynomial. For this reason, the development of quadrature rules that avoid sub-tessellation and optimize the number of function evaluations is an active research field. Several approaches have been proposed; in particular, we mention [145, 126, 105, 146], for example. Other approaches are represented by the Moment Fitting Equation technique, firstly proposed in [116], for the construction of quadrature rules on polygons featuring the same symmetry as the regular hexagon. The key idea here is, starting from a quadrature rule on the integration domain which integrates exactly a class of basis functions for a desired function space, an iterative node elimination procedure is then applied under an exactness constraint. This leads to the definition of a new quadrature rule where the number of function evaluations is optimized. Further improvements of the moment fitting equation algorithm can also be found in [123] and [138], see also [124] for a generalization to more general convex and non-convex polytopic domains. The main drawback of the moment fitting approach is the need to store the resulting nodes and weights for every polytope, which severely affects memory efficiency when applied to finite element approximations. An alternative approach designed to overcome the limitations of the sub-tessellation and the moment fitting equation methods is based on employing the generalized version of Stokes' theorem; with this approach, the integral over a generic domain is reduced to an integration over its boundary; we refer to [143] for further details. Following this idea, Sommariva and Vianello proposed in [135] a quadrature rule where, if an $x$ - or $y$-primitive of the integrand is available (as for bivariate polynomial functions), the integral over the polygon is reduced to a sum of line integrations over its edges, each of which is then computed exactly with a Gaussian one dimensional quadrature rule. The authors also generalized this approach to the more general case when the primitive is not known. While this algorithm does not directly require a sub-tessellation of the polygon, a careful choice of the parameters in the proposed formula leads to a quadrature rule that can be viewed as a particular 
sub-partition of the polygon itself. Moreover, in this case it is not possible to guarantee that all of the quadrature points lie inside the domain of integration. An alternative approach, proposed by Lassere in [114], provides a very efficient formula for the integration of homogeneous functions over convex polytopes. Here, the essential idea is to exploit the generalized Stokes' theorem together with Euler's homogeneous function theorem, cf. [134], in order to reduce the integration over a polytope only to boundary evaluations. The main difference with respect to the work presented in [135] is the possibility to apply the same idea recursively, leading to a quadrature formula which exactly evaluates integrals over a polygon/polyhedron by employing only point-evaluations of the integrand and its partial derivatives at the vertices of the polytope. This technique has been recently extended to general convex and non-convex polytopes in [74]. In Section 3 we present an efficient quadrature free algorithm for the numerical approximation of integrals of polynomial functions over general polygonal/polyhedral elements that do not require an explicit construction of a sub-tessellation. The method extends the idea of $[114,74]$ and is based on successive application of Stokes' theorem; thereby, the underlying integral may be evaluated using only the values of the integrand and its derivatives at the vertices of the polytopic domain. To demonstrate the practical performance of this quadrature free method we present some numerical results obtained by the numerical computation of the stiffness and mass matrices arising from $h p$-version PolyDG discretization of second-order elliptic partial differential equations.

Section 4 focuses on the analysis of PolyDG methods for the numerical discretization of seismic wave propagation; that is the ground motion phenomenon induced by the passage of body waves radially from the source of earthquake energy released into the surrounding soil medium. In the context of numerical modeling of direct and inverse wave propagation phenomena, many contributions can be found in the literature, stimulated not only by geophysical problems but also from vibroacoustics, aeroacoustic, acoustics, and electromagnetics engineering applications $[51,50,70,111,82,149,144,80,89,102]$. Here, our target are large-scale seismological phenomena and groundmotion induced by seismic events. Seismic waves are elastic waves propagating within the Earth and along its surface as a result of an earthquake, or of an explosion. Seismic waves induce a vibratory ground-motion in the area surrounding the seismic source. From the mathematical viewpoint, the propagation of seismic waves in a (visco)elastic heterogeneous material can be modeled by means of the elastodynamics equation. In order to solve the elastodynamics equation based on employing a finite element based numerical scheme, a number of distinguishing challenges have to simultaneously be taken into account which reflect the key features required by the numerical scheme: accuracy, geometric flexibility and scalability. High-order accuracy is mandatory in order to correctly approximate wave velocities, i.e., to keep as low as possible both the numerical dissipation and dispersion. Geometric flexibility is mandatory since within earthquake engineering the computational domain usually features complicated geometrical details, as well as sharp contrasts in the media. Finally, for real earthquake models, the size of the excited body is very large compared to the wave lengths of interest: this typically leads to numerical models featuring hundred of millions of unknowns, and therefore massively parallel scalable algorithms are required. Within the context of numerical methods for the approximation of the elastodynamics equation in computational seismology, spectral element methods are one of the most successfully employed tools, in particular for large scale applications; see, for example, [112, 113, 52, 93, 100]. To enhance the flexibility of spectral element methods, in recent years DG and DG spectral element (DGSE) methods has been extensively used for elastic waves propagation, see e.g. [132, 131, 83, 110, 91, 29, 121, 6, 92, 125, 25, 11, 10], and [17] for an overview on the numerical modeling of seismic waves by DGSE methods. Given their local nature, DG methods are particularly well suited to deal with highly heterogeneous media, or in soil-structure interaction problems, where local refinements are needed to resolve the different spatial scales [120]. In the context of time integration of the (second-order) ordinary differential systems stemming from spatial discretization of second-order hyperbolic partial differential equations we also mention the DG time-integration scheme of [27]. Very recently, also PolyDG methods have been shown to be perfectly suited to reduce the complexity of modelling wave propagation problems. Indeed, on the one hand they further enhance the geometric flexibly offered by 'classical' DG schemes on simplicial/tensor-product meshes, allowing for grids composed by arbitrarily shaped elements, with possibly degenerating faces, thus reducing the computational costs related to the process of grid generation, while maintaining the same degree of accuracy. On the other hand, they guarantee lower dispersion errors compared to classical DG schemes on simplicial/tensor-product grids of comparable granularity, see [26].

Section 5 is concerned with the numerical approximation of Darcy flows through porous media enclosing networks of fractures. The focus is on presenting a unified design and analysis of PolyDG methods on general polytopic meshes with possibly degenerating edges/faces. The problem of developing efficient numerical methods for fractured reservoir simulations has received increasing attention in the past decades, being fundamental in many energy and environmental 
engineering applications, such as water resources management, oil migration tracing, isolation of radioactive waste and groundwater contamination, for example. Fractures are regions of the porous medium featuring a different porous structure, so that they usually have a strong impact on the flow, possibly acting as barriers for the fluid (when they are filled with low permeable material), or as preferential paths (when their permeability is higher than that of the surrounding medium). Moreover, fractures are characterised by a very small width compared to their length and to the size of the domain. For this reason, one popular modelling choice consists in treating them as $(d-1)$-dimensional interfaces between $d$-dimensional porous matrices, $d=2,3$. The development of this kind of reduced models has been addressed for single-phase flows in several works, see, e.g., $[3,2,118,98]$. We will refer mainly to the model described in [118], see also [81], which considers the simplified case of a single, non-immersed fracture. Here, the flow in the porous medium (bulk) is assumed to be governed by Darcy's law and a suitable reduced version of the law is formulated also on the surface modelling the fracture. Physically consistent coupling conditions are then added (in strong form) to account for the exchange of fluid between the fracture and the porous medium. We remark that this model is able to handle both fractures with low and large permeability. Even if the use of this kind of dimensionally reduced models avoids the need for extremely refined grids inside the fracture domains, in realistic cases, the construction of a computational grid aligned with the fractures is still a major issue. For example, fractured oil reservoirs can feature thousands of fractures, which are often intersecting with small angles or nearly coincident [96]. In line with the discussion above, our aim is then to take advantage of the intrinsic geometric flexibility of PolyDG methods for the approximation of the coupled bulk-fracture problem, thus avoiding the limitations imposed by standard finite element methods. We also point out that various other numerical methods supporting polytopic elements have been employed in the literature for the approximation of this problem. In particular, we mention $[18,96]$, where a mixed approximation based on Mimetic Finite Differences has been explored; the works [48, 47], where a framework for treating flows in Discrete Fracture Networks based on the Virtual Element Method has been introduced, and [71], where the Hybrid High-Order method has been employed. We also mention that an alternative strategy consists in the use of nonconforming discretizations. Here, the bulk grid can be chosen fairly regular since fractures are allowed to arbitrarily cut it. We refer to [81, 99, 94] for the use of the eXtended Finite Element Method and to [61] for the Cut Finite Element Method. Notice that the geometric flexibility of PolyDG methods illustrated above is not the only motivation to employ these kinds of techniques for addressing this problem. Another important issue is that the discontinuous nature of the solution at the matrix-fracture interface is intrinsically captured in the choice of the discrete spaces. Moreover, coupling conditions between bulk and fracture can be easily reformulated using jump and average operators (basic tools for the construction of DG methods) and then naturally embedded in the variational formulation. Furthermore, employing the abstract setting, based on the flux-formulation, introduced in [33] for the unified analysis of all DG methods present in the literature, it is possible to introduce a unified framework where, according to the desired approximation properties of the model, one may resort to either a primal or mixed approximation for the problem in the bulk, as well as to a primal or mixed approximation for the problem in the fracture network. In particular, the primal discretizations are obtained using the Symmetric Interior Penalty DG method [148, 32], whereas the mixed discretizations are based on employing the Local DG (LDG) method of [79], both in their generalization to polytopic grids. Finally, we point out that, even if not addressed here, our formulation can be extended to the case of networks of intersecting fractures, cf. [15] and Section 5.4.

\section{Theoretical framework of PolyDG methods}

In this section we introduce the necessary notation and key analytical results required for the definition and analysis of PolyDG approximations. In particular, we summarize the main theoretical results concerning this class of methods contained in $[67,65,13,63,65]$, where an $h p$-version interior penalty PolyDG method for the numerical approximation of elliptic problems on polytopic meshes has been proposed and analysed. The exploitation of grids consisting of general polytopic elements poses a number of key challenges. Indeed, in contrast to the case when standard-shaped elements are employed, polytopes may admit an arbitrary number of faces/edges and the measure of these faces/edges may potentially be much smaller than the measure of the element itself. In $[12,67,13]$ it is assumed that the number of edges/faces of each mesh element is uniformly bounded. In $[63,65]$ this assumption is no longer required (i.e., elements with an arbitrary number of possibly degenerating faces/edges are admitted). However, this comes at the cost of adding an assumption (see Section 2 below) that may be regarded as the natural generalization to polytopic grids of the classical shape-regularity assumption [65]. For ease of presentation, we adopt the setting of [63, 65]; for the 
generalization to other classes of polytopic meshes, we refer to the recent article [62]. In particular, in Section 2.1, we introduce the notation related to the discretization of domains using polytopic elements and state the regularity assumptions on the meshes. In Section 2.2 we define the DG discrete spaces and introduce standard jump and average operators. Finally, in Section 2.3, starting from the mesh assumption of Section 2.1, we state trace inverse inequalities and approximation results for general polytopic elements that are sensitive to the type of edge/face degeneracy described above. We also remark that the capability of the method of handling faces with arbitrarily small measure is intimately related to the correct choice of the discontinuity-penalization function, which will be introduced in the following sections.

We will employ the following notation. For an open, bounded domain $D \subset \mathbb{R}^{d}, d=2,3$, we denote by $H^{s}(D)$ the standard Sobolev space of order $s$, for a real number $s \geq 0$. For $s=0$, we write $L^{2}(D)$ in lieu of $H^{0}(D)$. The usual norm on $H^{s}(D)$ is denoted by $\|\cdot\|_{H^{s}(D)}$ and the usual seminorm by $|\cdot|_{H^{s}(D)}$. We denote the corresponding Sobolev spaces of vector-valued functions and symmetric tensors by $\boldsymbol{H}^{m}(\Omega)=\left[H^{m}(D)\right]^{d}, \mathscr{H}^{m}(D)=\left[H^{m}(D)\right]_{\mathrm{sym}}^{d \times d}, d=2,3$, respectively. We also introduce the standard space $H_{\text {div }}(D)=\left\{\mathbf{v}: D \rightarrow \mathbb{R}^{d}:\|\mathbf{v}\|_{L^{2}(D)}+\|\nabla \cdot \mathbf{v}\|_{L^{2}(D)}<\infty\right\}$. Given a decomposition of the domain into a computational mesh $\mathscr{T}_{h}$, we denote by $H^{s}\left(\mathscr{T}_{h}\right)$ the standard broken Sobolev space, equipped with the broken norm $\|\cdot\|_{s, \mathscr{T}_{h}}$. Furthermore, we denote by $\mathbb{P}_{k}(D)$ the space of polynomials of total degree less than or equal to $k \geq 1$ on $D$. The symbols $\lesssim$ and $\gtrsim$ will signify that the inequalities hold up to multiplicative constants that are independent of the discretization parameters, but might depend on the physical parameters of the underlying problem.

\subsection{Grid assumptions}

Following $[67,65,13]$, we introduce the notation related to the subdivision of the computational domain $\Omega \subset \mathbb{R}^{d}$, $d=2,3$, by means of polytopic meshes. We consider classes of meshes $\mathscr{T}_{h}$ made of disjoint open polygonal/polyhedral elements $E$. For each element $E \in \mathscr{T}_{h}$, we denote by $|E|$ its measure, $h_{E}$ its diameter and we set $h=\max _{E \in \mathscr{T}_{h}} h_{E}$. With the aim of handling hanging nodes, we introduce the concept of mesh interfaces, which are defined as the intersection of the $(d-1)$-dimensional facets of two neighbouring elements. We need now to distinguish between the case when $d=3$ and $d=2$ :

- when $d=3$, each interface consists of a general polygon, which we assume may be decomposed into a set of coplanar triangles. We assume that a sub-triangulation of each interface is provided and we denote the set of all these triangles by $\mathscr{F}_{h}$. We then use the terminology face to refer to one of the triangular elements in $\mathscr{F}_{h}$;

- when $d=2$, each interface simply consists of a line segment, so that the concepts of face and interface are in this case coincident; however, we still denote by $\mathscr{F}_{h}$ the set of all faces.

Here, we note that $\mathscr{F}_{h}$ is always defined as a set of $(d-1)$-dimensional simplices (triangles or line segments).
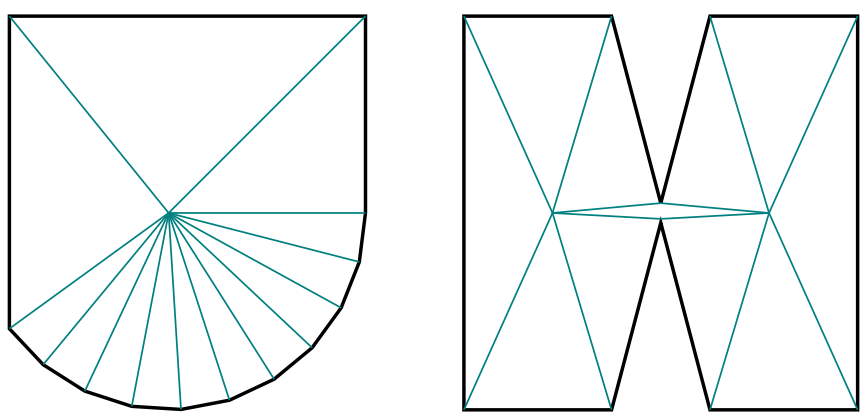

Fig. 2 Two examples of polytopic-regular elements as in Definition 1. Here, all the triangles $S_{E}^{F}$ (coloured in teal) have height of size comparable to the diameter $h_{E}$. Note also that the element on the right is not covered by the union of the simplices.

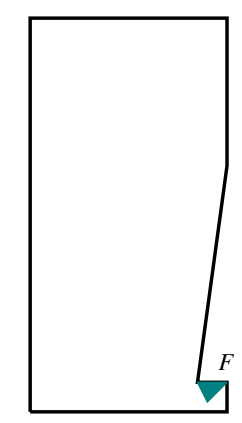

Fig. 3 Example of an element that violates polytopic-regularity: the shape of the polygon does not allow for the definition of a triangle $S_{E}^{F}$ with base $F$ whose height is comparable to the diameter $h_{E}$. 
In order to introduce the PolyDG formulation, it is useful to further subdivide the set $\mathscr{F}_{h}$ into

$$
\mathscr{F}_{h}=\mathscr{F}_{h}^{I} \cup \mathscr{F}_{h}^{B},
$$

where $\mathscr{F}_{h}^{I}$ is the set of interior faces and $\mathscr{F}_{h}^{B}$ is the set of faces lying on the boundary of the domain $\partial \Omega$. Moreover, if $\partial \Omega$ is split into the Dirichlet boundary $\Gamma_{D}$ and the Neumann boundary $\Gamma_{N}$, we will further decompose the set $\mathscr{F}_{h}^{B}=\mathscr{F}_{h}^{D} \cup \mathscr{F}_{h}^{N}$, where $\mathscr{F}_{h}^{D}$ and $\mathscr{F}_{h}^{N}$ are the boundary faces contained in $\Gamma_{D}$ and $\Gamma_{N}$, respectively. Implicit in this definition is the assumption that the mesh $\mathscr{T}_{h}$ conforms to the partition of $\partial \Omega$.

Next, we outline the key assumptions that the underlying polytopic mesh $\mathscr{T}_{h}$ needs to satisfy in order to derive suitable inverse inequalities and approximation results. To this end, we write $S_{E}^{F}$ to denote a $d$-dimensional simplex contained in $E$ which shares a specific face $F \subset \partial E, F \in \mathscr{F}_{h}$. With this notation we introduce the following definition.

Definition 1. A family of meshes $\left\{\mathscr{T}_{h}\right\}_{h}$ is said to be polytopic-regular if, for any $h$ and for any $E \in \mathscr{T}_{h}$, there exists a set of non-overlapping (not necessarily shape-regular) $d$-dimensional simplices $\left\{S_{E}^{F}\right\}_{F \subset \partial E}$ contained in $E$, such that for all faces $F \subset \partial E$, the following condition holds

$$
h_{E} \lesssim \frac{d\left|S_{E}^{F}\right|}{|F|},
$$

where the hidden constant is independent of the discretization parameters, the number of faces of the element, and the face measure.

We remark that the union of simplices $\left\{S_{E}^{F}\right\}_{F \subset \partial E}$ does not have to cover, in general, the whole element $E$, that is $\cup_{F \subset \partial E} \bar{S}_{E}^{F} \subseteq \bar{E}$, see Figure 2 for an example. We also stress that this definition does not require any restriction on either the number of faces per element or their relative measure. In particular, it allows the size of a face $|F|, F \subset \partial E$, to be arbitrarily small compared to the diameter of the element $h_{E}$, provided that the height of the corresponding simplex $S_{E}^{F}$ is comparable to $h_{E}$. Figure 2 shows two examples of elements belonging to a polytopic-regular mesh, while Figure 3 shows an element which may not satisfy the definition, for example, when the length of the vertical section of the boundary in the lower right-hand corner tends to zero at a faster rate than the mesh size $h$. We refer to [65] for more details.

Assumption 2 We assume that the family of meshes $\left\{\mathscr{T}_{h}\right\}_{h}$ is uniformly polytopic-regular.

This assumption will allow us to state the inverse trace estimate (1) below. The next definition and assumption are instrumental for the validity of the approximation results (2) below.

Definition 3. $[67,65,13,63,65]$ A covering $\mathscr{T}_{\#}=\left\{T_{E}\right\}$ related to the polytopic mesh $\mathscr{T}_{h}$ is a set of shape-regular $d$-dimensional simplices $T_{E}$, such that for each $E \in \mathscr{T}_{h}$, there exists a $T_{E} \in \mathscr{T}_{\#}$ such that $E \subsetneq T_{E}$.

Assumption 4 [67, 65, 13,63,65] There exists a covering $\mathscr{T}_{\#}$ of $\mathscr{T}_{h}$ (see Definition 3 ) and a positive constant $O_{\Omega}$, independent of the mesh parameters, such that

$$
\max _{E \in \mathscr{T}_{h}} \operatorname{card}\left\{E^{\prime} \in \mathscr{T}_{h}: E^{\prime} \cap T_{E} \neq \emptyset, T_{E} \in \mathscr{T}_{\#} \text { s.t. } E \subset T_{E}\right\} \leq O_{\Omega},
$$

and $h_{T_{E}} \lesssim h_{E}$ for each pair $E \in \mathscr{T}_{h}$ and $T_{E} \in \mathscr{T}_{\#}$, with $E \subset T_{E}$.

Assumption 4 implies that, when the computational mesh $\mathscr{T}_{h}$ is refined, the amount of overlap present in the covering $\mathscr{T}$ remains bounded.

\subsection{PolyDG discrete spaces}

Given a polytopic mesh partition $\mathscr{T}_{h}$ of the domain $\Omega$, the corresponding scalar, vector-valued and symmetric tensor-valued discontinuous finite element spaces are defined as 


$$
\begin{aligned}
Q_{h}^{D G} & =\left\{q_{h} \in L^{2}(\Omega):\left.q\right|_{E} \in \mathbb{P}_{p_{E}}(E) \forall E \in \mathscr{T}_{h}\right\}, \\
\mathbf{W}_{h}^{D G} & =\left\{\mathbf{w} \in\left[L^{2}(\Omega)\right]^{d}:\left.\mathbf{w}\right|_{E} \in\left[\mathbb{P}_{p_{E}}(E)\right]^{d} \forall E \in \mathscr{T}_{h}\right\}, \\
\mathscr{W}_{h}^{D G} & =\left\{\mathbf{w} \in\left[L^{2}(\Omega)\right]_{\mathrm{sym}}^{d \times d}:\left.\mathbf{w}\right|_{E} \in\left[\mathbb{P}_{p_{E}}(E)\right]_{\mathrm{sym}}^{d \times d} \forall E \in \mathscr{T}_{h}\right\},
\end{aligned}
$$

where we assume that $p_{E} \geq 1$ for all $E \in \mathscr{T}_{h}$. To avoid technicalities, for the analysis, we assume that a local bounded variation property holds for both the polynomial approximation degrees and the local mesh sizes, cf. [127].

Remark 1. From the implementation point of view, an essential feature of DG methods is that the local elemental polynomial spaces can be defined in the physical space, without the need to introduce a mapping to a reference element, as is typically necessary for classical finite element methods. This allows DG methods to naturally deal with general polytopic elements with polynomial degrees varying from one element to the other. A possible approach for the definition of the basis functions was first proposed in [67], based on the definition of the polynomial spaces over suitably defined bounding boxes of each polytopic element. More precisely, given an element $E \in \mathscr{T}_{h}$, we can define its (for example) Cartesian bounding box $B_{E}$, such that the sides of $B_{E}$ are aligned with the Cartesian axes and $\bar{E} \subseteq \bar{B}_{E}$. On the Cartesian bounding box $B_{E}$, we can then define a standard polynomial space, employing, for example, tensor-product Legendre polynomials. Finally, the polynomial basis over the general polytopic element may be defined by simply restricting the support of the basis functions to $E$; we refer to [65] for further details. We also mention that another key aspect related to the implementation of DG methods is the design of efficient numerical integration schemes over polytopic elements; this issue will be addressed in detail in the forthcoming Section (3.1), where a quadrature-free approach for the efficient integration of polynomial functions over polytopic domains will be discussed, following the recent work [22].

In order to efficiently deal with discontinuous functions, we now introduce average and jump operators on a face, which play a central role in the design and analysis of all DG methods [33]. Let $F \in \mathscr{F}_{h}^{I}$ be an interior face shared by the elements $E^{ \pm}$. We define $\mathbf{n}^{ \pm}$to be the unit normal vectors on $F$ pointing exterior to $E^{ \pm}$, respectively. Then, for sufficiently regular scalar-valued, vector-valued, and tensor-valued functions $q, \mathbf{v}$, and $\tau$, respectively, we define the standard average $\{\cdot\}$ and jump $\llbracket \cdot \rrbracket$ operators on $F$ as

$$
\begin{array}{rlrl}
\{q\} & =\frac{1}{2}\left(q^{+}+q^{-}\right), & & \llbracket q \rrbracket=q^{+} \mathbf{n}^{+}+q^{-} \mathbf{n}^{-}, \\
\{\mathbf{v}\}=\frac{1}{2}\left(\mathbf{v}^{+}+\mathbf{v}^{-}\right), & & \llbracket \mathbf{v} \rrbracket=\mathbf{v}^{+} \cdot \mathbf{n}^{+}+\mathbf{v}^{-} \cdot \mathbf{n}^{-}, \\
\{\tau\}=\frac{1}{2}\left(\tau^{+}+\tau^{-}\right), & \llbracket \tau \rrbracket=\tau^{+} \mathbf{n}^{+}+\tau^{-} \mathbf{n}^{-},
\end{array}
$$

where the subscript \pm on $q, \mathbf{v}$, and $\tau$ denote the respective traces of the functions on $F$ restricted to $E^{ \pm}$, respectively. To tackle elastic wave propagation phenomena, we also need the following jump operator for a sufficiently regular vector-valued function $\mathbf{v}$ :

$$
\llbracket \mathbf{v} \rrbracket=\mathbf{v}^{+} \odot \mathbf{n}^{+}+\mathbf{v}^{-} \odot \mathbf{n}^{-},
$$

where $\mathbf{v} \odot \mathbf{n}=\left(\mathbf{v} \mathbf{n}^{\top}+\mathbf{n v}^{\top}\right) / 2$. Notice that with the above definition $\llbracket \mathbf{v} \rrbracket \rrbracket$ is a $d \times d$ symmetric tensor. On a boundary face $F \in \mathscr{F}_{h}^{B}$ we set analogously $\{q\}=q, \llbracket q \rrbracket=q \mathbf{n},\{\mathbf{v}\}=\mathbf{v}, \llbracket \mathbf{v} \rrbracket=\mathbf{v} \cdot \mathbf{n}, \llbracket \mathbf{v} \rrbracket=\mathbf{v} \odot \mathbf{n},\{\tau\}=\tau$, and $\llbracket \tau \rrbracket=\tau \mathbf{n}$, where $\mathbf{n}$ is the outward unit normal vector on $\partial \Omega$, cf. $[34,33]$. For future use, we remark that on every $F \in \mathscr{F} h$ we can use the definition of jump and average operators to write

$$
\llbracket q \mathbf{v} \rrbracket=\llbracket \mathbf{v} \rrbracket\{q\}+\{\mathbf{v}\} \cdot \llbracket q \rrbracket .
$$

We also recall the identity:

$$
\sum_{E \in \mathscr{T}_{h}} \int_{\partial E} q \mathbf{v} \cdot \mathbf{n}_{E}=\int_{\mathscr{F}_{h} I \mathscr{F}_{h}^{B}}\{\mathbf{v}\} \cdot \llbracket q \rrbracket+\int_{\mathscr{F}_{h}^{I}} \llbracket \mathbf{v} \rrbracket\{q\},
$$

cf. [32], where we have used the compact notation $\int_{\mathscr{F}_{h}}=\sum_{F \in \mathscr{F}_{h}} \int_{F}$. 


\subsection{Trace inverse estimates on polytopic elements}

Trace inverse estimates are one of the key tools employed to study the stability and error analysis of DG-methods: they bound the norm of a polynomial on an element's face/edge by the norm on the element itself. In particular, Lemma 1 is required to establish the stability of the PolyDG approximation of second-order elliptic partial differential equations. Trace inverse estimates on polytopic elements are obtained under the polytopic-regular Assumption 2 as in [63], Lemma 4.1, and [21,65]; the proof is reported here for completeness.

Lemma 1. Let $E$ be a polytope satisfying Assumption 2 and let $q \in \mathbb{P}_{p_{E}}(E)$. Then, we have

$$
\|q\|_{L^{2}(\partial E)}^{2} \lesssim \frac{p_{E}^{2}}{h_{E}}\|q\|_{L^{2}(E)}^{2},
$$

where the hidden constant depends on the dimension d, but it is independent of the discretization parameters, i.e. the local mesh size $h_{E}$ and the local polynomial approximation degree $p_{E}$, and the number of faces that the element possesses.

Proof. The proof follows immediately if we apply "classical" $h p$-version inverse estimate valid for generic simplices, see, e.g., [147], to each simplex $S_{E}^{F} \subset E$, cf. Assumption 2, together with (1), i.e.,

$$
\begin{aligned}
\|q\|_{L^{2}(\partial E)}^{2} & =\sum_{F \subset \partial E}\|q\|_{L^{2}(F)}^{2} \lesssim p_{E}^{2} \sum_{F \subset \partial E} \frac{|F|}{\left|S_{E}^{F}\right|}\|q\|_{L^{2}\left(S_{E}^{F}\right)}^{2} \\
& \lesssim \frac{p_{E}^{2}}{h_{E}}\|q\|_{L^{2}\left(\bigcup_{F \subset \partial E} S_{E}^{F}\right)}^{2} \leq \frac{p_{E}^{2}}{h_{E}}\|q\|_{L^{2}(E)}^{2} .
\end{aligned}
$$

A crucial mathematical tool needed to study the a priori error analysis of PolyDG methods are $h p$-interpolation estimates. In $[67,65,13]$ standard results on simplices are extended to polytopic elements, based on considering appropriate coverings and submeshes consisting of $d$-dimensional simplices (where standard results can be applied) and using an appropriate extension operator. In [63] these results are further extended in order to be successfully applied also in the case when the number of edges/faces is unbounded. Here, we recall the results contained in $[67,65,13,63,65]$.

Let $\mathscr{E}: H^{s}(\Omega) \rightarrow H^{s}\left(\mathbb{R}^{d}\right), s \geq 0$, be the continuous extension operator introduced by Stein in [136], such that $\left.\mathscr{E}(q)\right|_{\Omega}=q$ and $\|\mathscr{E} q\|_{H^{s}\left(\mathbb{R}^{d}\right)} \lesssim\|q\|_{H^{s}(\Omega)}$. Based on the existence of a suitable covering of the polytopic mesh (see Definition 3)), we can state the following approximation result.

Lemma 2. $[67,13,63,65]$ Assume that Assumptions 2 and 4 are satisfied. Given $E \in \mathscr{T}_{h}$, let $T_{E} \in \mathscr{T}_{\#}$ be the corresponding simplex such that $E \subset T_{E}$ (see Definition 3). For $q \in L^{2}(\Omega)$, such that $\left.\mathscr{E} q\right|_{T_{E}} \in H^{r_{E}}\left(T_{E}\right)$, for some $r_{E} \geq 0$, there exists a sequence of approximations $\Pi_{E}^{p_{E}} q \in \mathbb{P}_{p_{E}}(E), p_{E}=0,1,2, \ldots$, of $q$ satisfying

$$
\left\|q-\Pi_{E}^{p_{E}} q\right\|_{H^{m}(E)} \lesssim \frac{h_{E}^{s_{E}-m}}{p_{E}^{r_{E}-m}}\|\mathscr{E} q\|_{H^{r} E\left(T_{E}\right)}, \quad 0 \leq m \leq r_{E}
$$

Moreover, if $r_{E} \geq 1+d / 2$,

$$
\left\|q-\Pi_{E}^{p_{E}} q\right\|_{L^{2}(\partial E)} \lesssim \frac{h_{E}^{s_{E}-1 / 2}}{p_{E}^{r_{E}-1 / 2}}\|\mathscr{E} q\|_{H^{r_{E}\left(T_{E}\right)}} .
$$

Here, $s_{E}=\min \left(p_{E}+1, r_{E}\right)$ and the hidden constants depend on the shape-regularity of $T_{E}$, but are independent of $q$, $h_{E}, p_{E}$ and the number of faces per element.

Proof. See [67] for a detailed proof of (6) and [63] for the proof of (7).

We note that the inequalities (5) and (7) hold on the whole boundary of $E$, and not just on one of its edges/faces; this is of fundamental importance in the analysis when considering elements that contain an arbitrary number of faces. 


\section{Computing integrals over polytopic mesh elements and mesh interfaces}

In this section we review the quadrature free approach for the efficient computation of the volume/face integral terms appearing in PolyDG methods. We point out that our approach is completely general and can be directly applied to other discretization schemes, such as VEM, HHO, Hybridizable DG, and MFD, for example. We present the main idea of the algorithm and show that our integration approach leads to a considerable improvement in the computational performance compared to classical quadrature algorithms based on sub-tessellation, in both two- and three-dimensions.

\subsection{Quadrature free algorithm}

First, we recall the idea introduced by Chin, Lasserre, and Sukumar in [74] for the integration of homogeneous function $\mathrm{g}$ over a polytopic domain $\mathscr{P}$, where

- $\mathscr{P} \subset \mathbb{R}^{d},=2,3$, is a closed polytope, whose boundary $\partial \mathscr{P}$ is defined by $m(d-1)$-dimensional faces $F_{i}, i=$ $1, \ldots, m$, cf. Figure 4 . Each face $F_{i}$ lies in a hyperplane $\mathscr{H}_{i}$ identified by a vector $\mathbf{a}_{i} \in \mathbb{R}^{d}$ and a scalar number $b_{i}$, such that

$$
\boldsymbol{x} \in \mathscr{H}_{i} \Longleftrightarrow \mathbf{a}_{i} \cdot \boldsymbol{x}=b_{i}, \quad i=1, \ldots, m .
$$

We observe that $\mathbf{a}_{i}, i=1, \ldots, m$, can be chosen as the unit outward normal vector to $F_{i}, i=1, \ldots, m$, respectively, relative to $\mathscr{P}$.

- $g: \mathscr{P} \rightarrow \mathbb{R}$ is a homogeneous function of degree $q \in \mathbb{R}$, i.e., for all $\lambda>0, g(\lambda \boldsymbol{x})=\lambda^{q} g(\boldsymbol{x})$ for all $\boldsymbol{x} \in \mathscr{P}$.

Euler's homogeneous function theorem [134] states that, if $g$ is a homogeneous function of degree $q \geq 0$, then the following identity holds:

$$
q g(\boldsymbol{x})=\nabla g(\boldsymbol{x}) \cdot \boldsymbol{x} \quad \forall \boldsymbol{x} \in \mathscr{P} .
$$

We point out that, in view of the application to polygonal/polyhedral finite element methods, we are interested in the integration of a particular class of homogeneous functions, namely polynomial homogeneous functions of the form

$$
g(\boldsymbol{x})=x_{1}^{k_{1}} x_{2}^{k_{2}} \cdots x_{d}^{k_{d}}, \quad \text { where } k_{n} \in \mathbb{N}_{0}, \text { for } n=1, \ldots, d,
$$

that is a homogeneous function of degree $q=k_{1}+\cdots+k_{d}$, and the general partial derivative $\frac{\partial g}{\partial x_{n}}$ is still a homogeneous function of degree $q-1$.

Next we recall the generalized Stokes' theorem, cf. [143]: given a generic vector field $\mathbf{X}: \mathscr{P} \rightarrow \mathbb{R}^{d}$, we have that

$$
\int_{\mathscr{P}}(\nabla \cdot \mathbf{X}(\boldsymbol{x})) g(\boldsymbol{x})+\int_{\mathscr{P}} \nabla g(\boldsymbol{x}) \cdot \mathbf{X}(\boldsymbol{x})=\int_{\partial \mathscr{P}} \mathbf{X}(\boldsymbol{x}) \cdot \mathbf{n}(\boldsymbol{x}) g(\boldsymbol{x})
$$

where $\mathbf{n}$ is the unit outward normal vector to $\mathscr{P}$. Selecting $\mathbf{X}=\boldsymbol{x}$ in (10), and employing (8), gives

$$
\int_{\mathscr{P}} g(\boldsymbol{x})=\frac{1}{d+q} \int_{\partial \mathscr{P}} \boldsymbol{x} \cdot \mathbf{n}(\boldsymbol{x}) g(\boldsymbol{x})=\frac{1}{d+q} \sum_{i=1}^{m} b_{i} \int_{F_{i}} g(\boldsymbol{x}) .
$$

Equation (11) states that the integral of a homogeneous function $g$ over a polytope $\mathscr{P}$ can be computed by integrating the same function over the boundary faces $F_{i} \subset \partial \mathscr{P}, i=1, \ldots, m$. By recursion, we can further reduce each term $\int_{F_{i}} g(\boldsymbol{x}), i=1, \ldots, m$, to the integration over $\partial F_{i}, i=1, \ldots, m$, respectively. To this end, Stokes' theorem needs to be applied on the hyperplane $\mathscr{H}_{i}, i=1, \ldots, m$, in which each $F_{i}, i=1, \ldots, m$, lies, respectively. In order to proceed, let $\gamma: \mathbb{R}^{d-1} \rightarrow \mathbb{R}^{d}$ be the function which expresses a generic point $\tilde{\mathbf{x}}=\left(\tilde{x}_{1}, \ldots, \tilde{x}_{d-1}\right)^{\top} \in \mathbb{R}^{d-1}$ as a point in $\mathbb{R}^{d}$ that lies on $\mathscr{H}_{i}, i=1, \ldots, m$, i.e.,

$$
\tilde{\mathbf{x}} \longmapsto \gamma(\tilde{\mathbf{x}})=\boldsymbol{x}_{0, i}+\sum_{n=1}^{d-1} \tilde{x}_{n} \mathbf{e}_{i n}, \quad \text { with } \mathbf{e}_{i n} \in \mathbb{R}^{d}, \mathbf{e}_{i n} \cdot \mathbf{e}_{i m}=\delta_{n m} .
$$



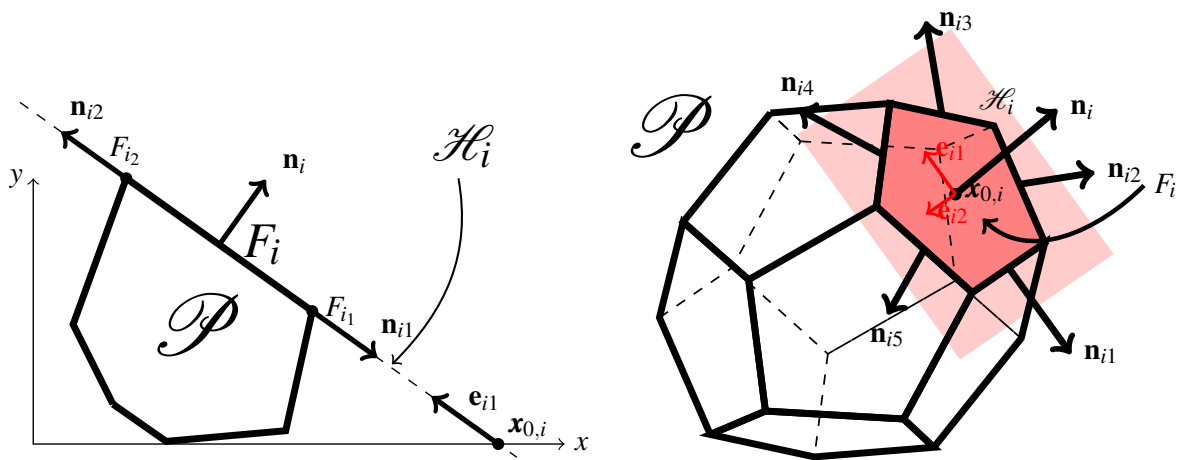

Fig. 4 Left: Example of a two-dimensional polytope $\mathscr{P}$ and its face $F_{i}$. The hyperplane $\mathscr{H}_{i}$ is defined by the local origin $\boldsymbol{x}_{0, i}$ and the vector $\mathbf{e}_{i 1}$. Right: the dodecahedron $\mathscr{P}$ with pentagonal faces and the face $F_{i} \subset \partial \mathscr{P}$ with unit outward normal vector $\mathbf{n}_{i}$. Here, $F_{i}$ has five edges $F_{i j}, j=1, \ldots, 5$, and five unit outward normal vectors $\mathbf{n}_{i j}, j=1, \ldots, 5$, lying on the plane $\mathscr{H}_{i}$. The hyperplane $\mathscr{H}_{i}$ is identified by the local origin $\boldsymbol{x}_{0, i}$ and the orthonormal vectors $\mathbf{e}_{i 1}, \mathbf{e}_{i 2}$. Figure taken from [22].

Here, $\boldsymbol{x}_{0, i} \in \mathscr{H}_{i}, i=1, \ldots, m$, is an arbitrary point which represents the origin of the coordinate system on $\mathscr{H}_{i}$, and $\left\{\mathbf{e}_{i n}\right\}_{n=1}^{d-1}$ is an orthonormal basis on $\mathscr{H}_{i}, i=1, \ldots, m$; see Figure 4 . We observe that $\boldsymbol{x}_{0, i}$ does not have to lie inside $F_{i}, i=1, \ldots, m$. By defining $\widetilde{F}_{i} \subset \mathbb{R}^{d-1}$ such that $\gamma\left(\widetilde{F}_{i}\right)=F_{i}, i=1, \ldots, m$, we obtain

$$
\int_{F_{i}} g(\boldsymbol{x})=\int_{\widetilde{F}_{i}} g(\gamma(\tilde{\mathbf{x}})), \quad i=1, \ldots, m .
$$

It is easy to prove that, writing $F_{i j} \subset \partial F_{i} j=1, \ldots, m_{i}$, to denote the vertices/edges of $F_{i}, i=1, \ldots, m$, for $d=2,3$, respectively, the following identity holds

$$
\widetilde{\mathbf{n}}_{i j}=\underline{\mathbf{E}}^{\top} \mathbf{n}_{i j}, \quad i=1, \ldots, m, j=1, \ldots, m_{i},
$$

where $\mathbf{n}_{i j}$ is the unit outward normal vectors to $F_{i j}$ lying in $\mathscr{H}_{i}, \underline{\mathbf{E}} \in \mathbb{R}^{d \times(d-1)}$, whose columns are the vectors $\left\{\mathbf{e}_{i n}\right\}_{n=1}^{d-1}, i=1, \ldots, m, \widetilde{F_{i j}} \subset \partial \widetilde{F}_{i}$ is the preimage of $F_{i j}$ with respect to the map $\gamma$, and $\widetilde{\mathbf{n}}_{i j}$ are the corresponding unit outward normal vectors; we refer to [22] for more details. Next we recall the following result.

Proposition 1. [22, Proposition 1] Let $F_{i}, i=1, \ldots, m$, be a face of the polytope $\mathscr{P}$, and let $F_{i j}, j=1, \ldots, m_{i}$, be the planar/straight faces/edges such that $\partial F_{i}=\cup_{j=1}^{m_{i}} F_{i j}$ for some $m_{i} \in \mathbb{N}$. Then, for any homogeneous function $g$, of degree $q \geq 0$, the following identity holds

$$
\int_{F_{i}} g(\boldsymbol{x})=\frac{1}{d-1+q}\left(\sum_{j=1}^{m_{i}} d_{i j} \int_{F_{i j}} g(\boldsymbol{x})+\int_{F_{i}} \boldsymbol{x}_{0, i} \cdot \nabla g(\boldsymbol{x})\right),
$$

where $d_{i j}$ denotes the Euclidean distance between $F_{i j}$ and $\boldsymbol{x}_{0, i}, \boldsymbol{x}_{0, i} \in \mathscr{H}_{i}$, is arbitrary, $i=1, \ldots, m$.

Using Proposition 1, together with equation (11), we obtain the following identity

$$
\int_{\mathscr{P}} g(\boldsymbol{x})=\frac{1}{d+q} \sum_{i=1}^{m} \frac{b_{i}}{d-1+q}\left(\sum_{j=1}^{m_{i}} d_{i j} \int_{F_{i j}} g(\boldsymbol{x})+\int_{F_{i}} \boldsymbol{x}_{0, i} \cdot \nabla g(\boldsymbol{x})\right)
$$

where we recall that $\partial \mathscr{P}=\cup_{i=1}^{m} F_{i}$ and $\partial F_{i}=\cup_{j=1}^{m_{i}} F_{i j}$, for $i=1, \ldots, m$. We point out that in two-dimensions, i.e., $d=2$, then $F_{i j}$ is a point and (12) states that the integral of $g$ on $\mathscr{P}$ can be computed by vertex-evaluations of the integrand plus a line integration of the partial derivative of $g$. If $d=3$ we can apply Stokes' Theorem recursively to $\int_{F_{i j}} g(\boldsymbol{x})$. We point out that, whenever $g$ is a homogeneous polynomial function of the form (9), so that the derivatives of $g(\cdot)$ are homogeneous polynomial functions as well, it is possible to recursively apply formula (12) to the terms involving the integration of the derivatives of $g$. With this observation in mind, we define the function that returns the integral of the polynomial $x_{1}^{k_{1}} \ldots x_{d}^{k_{d}}$ over $\mathscr{E}$ as 


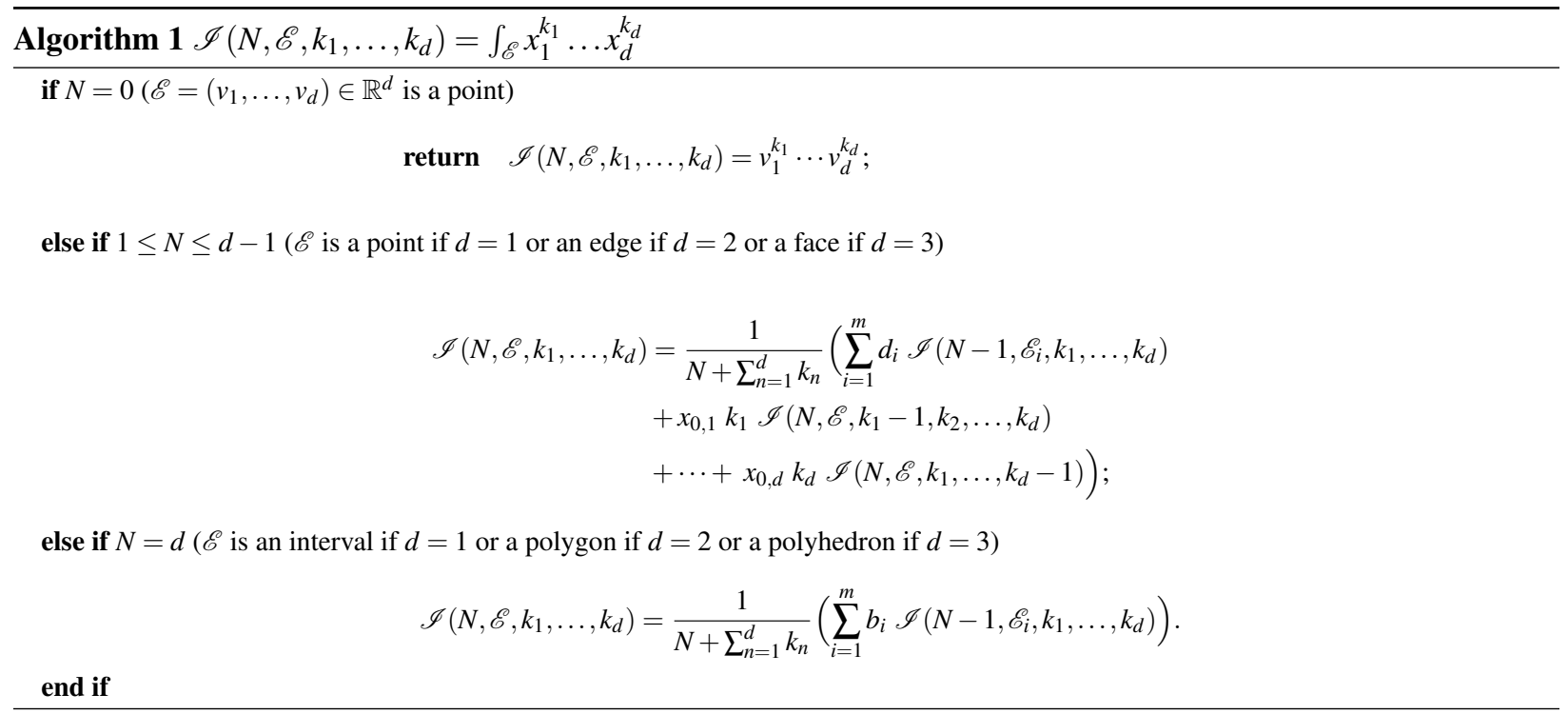

Table 1 Polytopic domains of integration $\mathscr{E}$ as a function of the dimension $d$, cf. Algorithm 1.

\begin{tabular}{c|c|c|c|c}
\hline & $N=3$ & $N=2$ & $N=1$ & $N=0$ \\
\hline$d=3$ & $\begin{array}{c}\mathscr{E}=\mathscr{P} \\
\text { is a polyhedron }\end{array}$ & $\begin{array}{c}\mathscr{E}=F_{i} \subset \partial \mathscr{P} \\
\text { is a polygon }\end{array}$ & $\begin{array}{c}\mathscr{E}=F_{i j} \subset \partial F_{i} \\
\text { is an edge }\end{array}$ & $\begin{array}{c}\mathscr{E}=F_{i j k} \subset \partial F_{i j} \\
\text { is a point }\end{array}$ \\
\hline$d=2$ & & $\begin{array}{c}\mathscr{E}=\mathscr{P} \\
\text { is a polygon }\end{array}$ & $\begin{array}{c}\mathscr{E}=F_{i} \subset \partial \mathscr{P} \\
\text { is an edge }\end{array}$ & $\begin{array}{c}\mathscr{E}=F_{i j} \subset \partial F_{i} \\
\text { is a point }\end{array}$ \\
\hline$d=1$ & & & $\begin{array}{c}\mathscr{E}=\mathscr{P} \\
\text { is an interval }\end{array}$ & $\begin{array}{c}\mathscr{E}=F_{i} \subset \partial \mathscr{P} \\
\text { is a point }\end{array}$ \\
\hline
\end{tabular}

$$
\mathscr{I}\left(N, \mathscr{E}, k_{1}, \ldots, k_{d}\right)=\int_{\mathscr{E}} x_{1}^{k_{1}} \ldots x_{d}^{k_{d}}
$$

where $\mathscr{E} \subset \mathbb{R}^{d}, d=2,3$, can be a $N$-polytopic domain of integration, with $N=1, \ldots, d, \partial \mathscr{E}=\cup_{i=1}^{m} \mathscr{E}_{i}$, where each $\mathscr{E}_{i} \subset$ $\mathbb{R}^{d}$ is a $(N-1)$-polytopic domain. When $N=d$ and $d=2,3, \mathscr{E}_{i}, i=1, \ldots, m$, will be an edge or a face, respectively; see Table 1 for details. According to Proposition 1, the recursive definition of the function $\mathscr{I}(\cdot, \cdot, \ldots, \cdot)$ is given in Algorithm 1. We point out that the computational complexity of Algorithm 1 depends in general on the number of recursive calls of the function $\mathscr{I}(\cdot, \cdot, \ldots, \cdot)$; a detail discussion on the FLOPS required by Algorithm 2 and on optimization strategies to improve the computational complexity of Algorithm 1 are discussed in [22]. Here, we just remark that in the context of employing the quadrature free approach within a polygonal finite element method, we are not interested in integrating a single monomial function, but instead an entire family of monomials, which, for example, form a basis for the space of polynomials of a given degree over a given polytopic element $E \in \mathscr{T}_{h}$. For example, when $d=2$, let us consider the evaluation of

$$
\int_{E} x^{k_{1}} y^{k_{2}} \quad \forall k_{1}, k_{2} \geq 0, \quad k_{1}+k_{2} \leq p
$$

As shown in [22], when employing Algorithm 1 with an with an optimal choice of the points which define the origin of the coordinate system on each element facet, the total number of FLOPs required for the computation of (13) is approximately $\mathscr{O}\left(p^{3}\right)$, as $p$ increases. To improve efficiency, an alternative approach, cf. Algorithms 2 and 3 , are based on the observation that, using the notation of Algorithm 1 , if the values of $\mathscr{I}\left(N-1, \mathscr{E}_{j}, k_{1}, \ldots, k_{d}\right), j=1, \ldots, m$, $\mathscr{I}\left(N, \mathscr{E}, k_{1}-1, \ldots, k_{d}\right) \ldots \mathscr{I}\left(N, \mathscr{E}, k_{1}, \ldots, k_{d}-1\right)$, for $1 \leq N \leq d-1$, in Algorithm 1 , have already been computed, then the computation of $\mathscr{I}\left(N, \mathscr{E}, k_{1}, \ldots, k_{d}\right)$ is extremely cheap. Indeed, since we must store the integrals of all the monomials on $E$ anyway, we can start by computing and storing $\int_{E} x^{k_{1}} y^{k_{2}}$ related to the lower degrees $k_{1}, k_{2}$ and $N=1$, then exploit these values in order to compute the integrals with higher degrees $k_{1}, k_{2}$ and higher dimension $N$ 

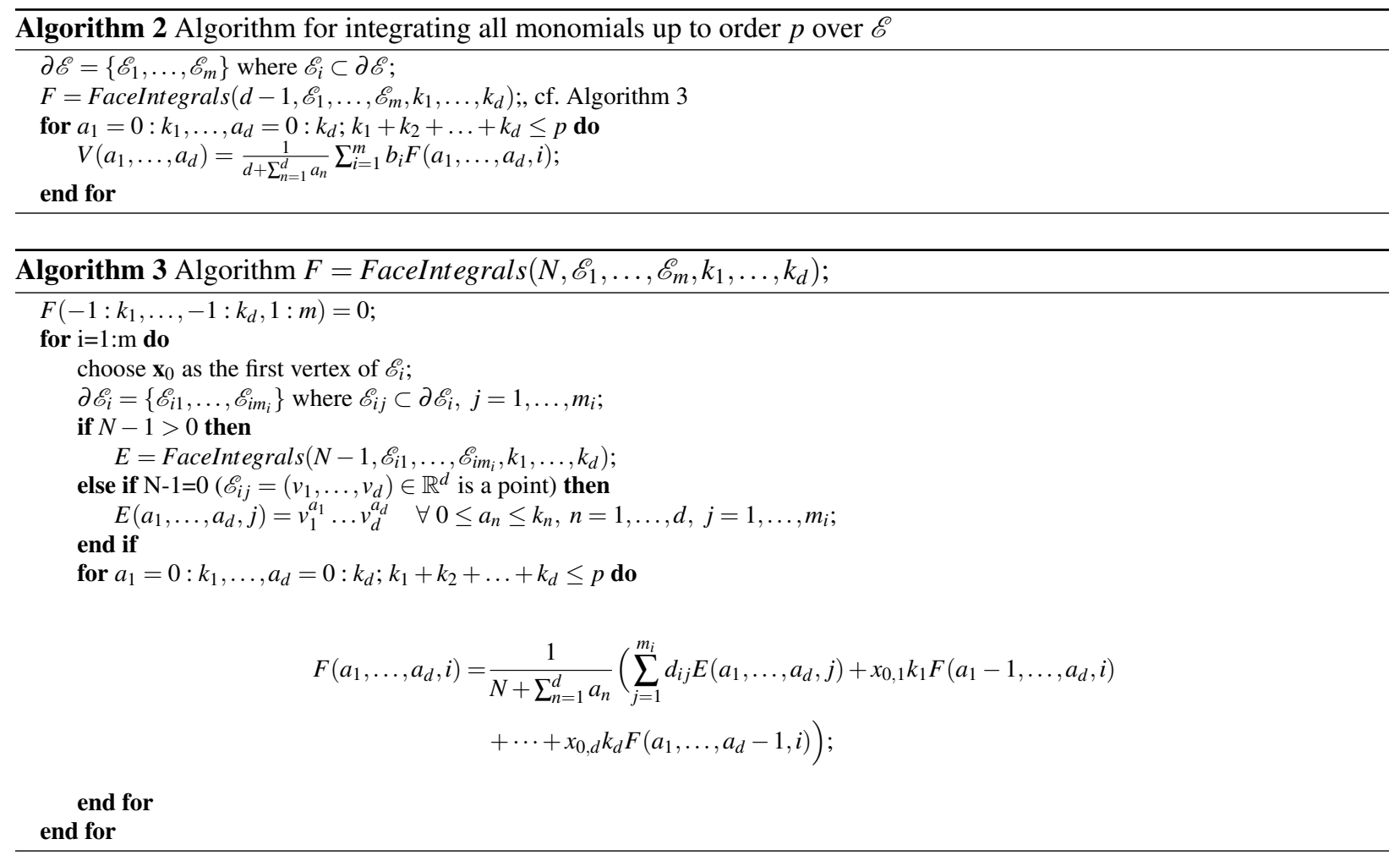

of the integration domain $\mathscr{E}$. We remark that, in Algorithm 3, $d_{i j}$ represents the Euclidean distance between $\mathscr{E}_{i j}$ and $\mathbf{x}_{0}, j=1, \ldots, m_{i j}$.

\subsection{Volume and interface integrals over polytopic mesh elements}

To fix the ideas, we restrict our discussion to the two-dimensional scalar case, but note that the three-dimensional and vector-/tensor-valued cases follow in a completely analogous manner. Let $\left\{\phi_{i}\right\}_{i=1}^{N_{h}}$ be a basis for the discrete space $Q_{h}^{D G}$ defined as in (2) whose dimension is $N_{h}$. For the construction of the discrete space $Q_{h}^{D G}$ we can exploit, for example, the approach presented in [67], based on employing polynomial spaces defined over the bounding box of each element, cf. Remark 1. More precisely, given an element $E \in \mathscr{T}_{h}$, we first construct the Cartesian bounding box $B_{E}$, such that $\bar{E} \subset \overline{B_{E}}$ and define a linear map between $\mathbf{F}_{E}: \hat{B} \rightarrow B_{E}$ such that

$$
\mathbf{F}_{E}: \hat{\boldsymbol{x}} \in \hat{B} \longmapsto \mathbf{F}_{E}(\hat{\boldsymbol{x}})=\mathbf{J}_{E} \hat{\boldsymbol{x}}+\mathbf{t}_{E},
$$

where $\hat{B}=(-1,1)^{d}$ and $\mathbf{J}_{E} \in \mathbb{R}^{d \times d}$ is the (diagonal) Jacobi matrix of the transformation, and $\mathbf{t}_{E} \in \mathbb{R}^{d}$ is the translation between the point $\mathbf{0} \in \hat{B}$ and the baricenter of the bounded box $B_{E}$, see Figure 5 .

We first discuss the application of Algorithm 2 for the efficient computation of the local volume integrals over polytopic mesh elements, focusing on the local mass and stiffness volume matrices defined as

$$
\mathbf{M}_{i, j}^{E}=\int_{\Omega} \phi_{i, E} \phi_{j, E}, \quad \mathbf{V}_{i, j}^{E}=\int_{\Omega} \nabla \phi_{i, E} \cdot \nabla \phi_{j, E}, \quad i, j=1, \ldots, N_{p_{E}}
$$

respectively, for all $E \in \mathscr{T}_{h}$. Here, $N_{p_{E}}$ is the dimension of the local discrete space, and $\phi_{i, E}$ and $\phi_{j, E}$ are the restriction to $E$ of $\phi_{i}$ and $\phi_{j}$, respectively. Employing the transformation $\mathbf{F}_{E}$ given in (14) we have for the mass matrix 
Fig. 5 Example of a polygonal element $E \in \mathscr{T}_{h}$, the relative bounding box $B_{E}$, the $\operatorname{map} \mathbf{F}_{E}$ and $\hat{E}=\mathbf{F}_{E}^{-1}(E)$. Figure taken from [22].

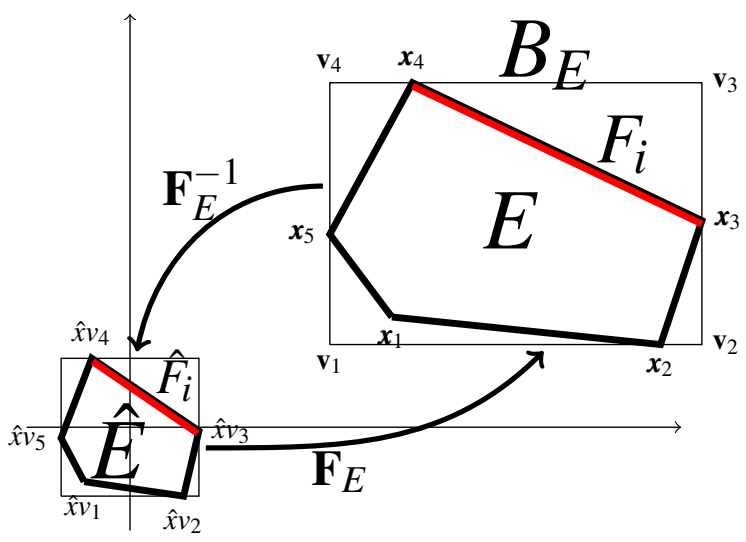

$\mathbf{M}_{i, j}^{E}=\int_{E} \phi_{i, E} \phi_{j, E}=\int_{\hat{E}} \hat{\phi}_{i} \hat{\phi}_{j}\left|\mathbf{J}_{E}\right|, \quad i, j=1, \ldots, N_{p_{E}}$,

where $\hat{E}=\mathbf{F}_{E}^{-1}(E) \subset \hat{B}$, see Figure 5, and the Jacobian of the transformation $\mathbf{F}_{E}$ is constant and is given by $\left|\mathbf{J}_{E}\right|=\left(\mathbf{J}_{E}\right)_{1,1}\left(\mathbf{J}_{E}\right)_{2,2}$, thanks to the definition of the map (14).

In order to employ Algorithm 2, we need to identify the coefficients of the homogeneous polynomial expansion for the function $\hat{\phi}_{i}(\hat{x}, \hat{y}) \hat{\phi}_{j}(\hat{x}, \hat{y})$. We can write, for example, any shape function $\hat{\phi}=\hat{\phi}_{i}(\hat{x}, \hat{y})$ as the product of onedimensional Legendre polynomial $\mathscr{L}_{i}$, i.e., $\hat{\phi}_{i}(\hat{x}, \hat{y})=\mathscr{L}_{i_{1}}(\hat{x}) \mathscr{L}_{i_{2}}(\hat{y})$, and each Legendre polynomial can be expanded as

$$
\mathscr{L}_{i_{1}}(\hat{x})=\sum_{m=0}^{i_{1}} C_{i_{1}, m} \hat{x}^{m}, \quad \mathscr{L}_{i_{2}}(\hat{y})=\sum_{n=0}^{i_{2}} C_{i_{2}, n} \hat{y}^{n}
$$

Therefore, we have

$$
\begin{aligned}
\mathbf{M}_{i, j}^{E} & =\int_{\hat{E}}\left(\sum_{m=0}^{i_{1}} C_{i_{1}, m} \hat{x}^{m}\right)\left(\sum_{n=0}^{i_{2}} C_{i_{2}, n} \hat{y}^{n}\right)\left(\sum_{s=0}^{j_{1}} C_{j_{1}, s} \hat{x}^{s}\right)\left(\sum_{r=0}^{j_{2}} C_{j_{2}, r} \hat{y}^{r}\right)\left|\mathbf{J}_{E}\right| \\
& =\int_{\hat{E}}\left(\sum_{k=0}^{i_{1}+j_{1}} \mathscr{C}_{i_{1}, j_{1}, k} \hat{x}^{k}\right)\left(\sum_{l=0}^{i_{2}+j_{2}} \mathscr{C}_{i_{2}, j_{2}, l} \hat{y}^{l}\right)\left|\mathbf{J}_{E}\right| \\
& =\sum_{k=0}^{i_{1}+j_{1}} \sum_{l=0}^{i_{2}+j_{2}} \mathscr{C}_{i_{1}, j_{1}, k} \mathscr{C}_{i_{2}, j_{2}, l}\left|\mathbf{J}_{E}\right| \int_{\hat{E}} \hat{x}^{k} \hat{y}^{l}
\end{aligned}
$$

where we have defined the compact notation

$$
\mathscr{C}_{i, j, k}=\sum_{n+m=k}\left(C_{i, n} C_{j, m}\right), \quad \text { for } 0 \leq i, j \leq p_{E}, \quad 0 \leq k \leq i+j,
$$

and where we stress that the coefficients $\mathscr{C}_{i, j, k}$ can be evaluated, once and for all, independently of the polygonal element $E$.

Concerning the general element of the volume matrix $\mathbf{V}_{i, j}^{E}$, cf. (15), we can proceed as before; indeed, following [22], we obtain

$$
\begin{aligned}
\mathbf{V}_{i, j}^{E}= & \sum_{k=0}^{i_{1}+j_{1}-2} \sum_{l=0}^{i_{2}+j_{2}} \mathscr{C}_{i_{1}, j_{1}, k}^{\prime} \mathscr{C}_{i_{2}, j_{2}, l}\left(\mathbf{J}_{E}^{-1}\right)_{1,1}^{2}\left|\mathbf{J}_{E}\right| \int_{\hat{E}} \hat{x}^{k} \hat{y}^{l} \\
& +\sum_{k=0}^{i_{1}+j_{1}} \sum_{l=0}^{i_{2}+j_{2}-2} \mathscr{C}_{i_{1}, j_{1}, k} \mathscr{C}_{i_{2}, j_{2}, l}^{\prime}\left(\mathbf{J}_{E}^{-1}\right)_{2,2}^{2}\left|\mathbf{J}_{E}\right| \int_{\hat{E}} \hat{x}^{k} \hat{y}^{l},
\end{aligned}
$$

where $\mathscr{C}_{i, j, k}$ is defined in (16), and 


$$
\mathscr{C}_{i, j, k}^{\prime}=\sum_{n+m=k} C_{i, n}^{\prime} C_{j, m}^{\prime}, \quad 1 \leq i, j \leq p_{E}, \text { for } 0 \leq k \leq i+j-2 .
$$

Here, $C_{i, n}^{\prime}=(n+1) C_{i, n+1}, C_{j, m}^{\prime}=(m+1) C_{j, m+1}$ are the expansion coefficients of the derivatives of the Legendre polynomials which are again computable independent of the element $E, E \in \mathscr{T}_{h}$, i.e.,

$$
\mathscr{L}_{0}^{\prime}(\hat{x})=0, \quad \mathscr{L}_{i}^{\prime}(\hat{x})=\sum_{m=0}^{i-1}(m+1) C_{i, m+1} \hat{x}^{m}=\sum_{m=0}^{i-1} C_{i, m}^{\prime} \hat{x}^{m}, \quad \text { for } i>0 .
$$

We next recall how to compute the key terms that arise in the interface integrals when PolyDG methods are employed for the numerical approximation of second-order partial differential equations. As before, we can transform the integral over a physical face $F \subset \partial E$ to the corresponding integral over the face $\hat{F}=\mathbf{F}_{E}^{-1}(F) \subset \partial \hat{E}$ on the reference rectangular element $\hat{E}$. From the definition of the jump and average operators, cf. (3), on each face $F \in \mathscr{F}{ }_{h}^{I}$ shared by the elements $E^{+}$and $E^{-}$we need to assemble contributions of the form

$$
\begin{aligned}
\mathbf{S}_{i, j}^{ \pm / \mp}=\int_{F}\left(\phi_{i, E^{ \pm}} \mathbf{n}^{ \pm}\right) \cdot\left(\phi_{j, E^{\mp}} \mathbf{n}^{\mp}\right), & i=1, \ldots, N_{p_{E^{ \pm}}}, & j=1, \ldots, N_{p_{E^{\mp}}}, \\
\mathbf{I}_{i, j}^{ \pm / \mp}=\frac{1}{2} \int_{F}\left(\nabla \phi_{i, E^{ \pm}} \cdot \mathbf{n}^{ \pm}\right) \phi_{j, E^{\mp}}, & i=1, \ldots, N_{p_{E^{ \pm}}}, & j=1, \ldots, N_{p_{E^{\mp}}} .
\end{aligned}
$$

Analogously, on the boundary face $F \in \mathscr{F}_{h}^{B}$ belonging to $E^{+} \in \mathscr{T}_{h}$ we only have to compute

$$
\mathbf{S}_{i, j}^{+/+}=\int_{F} \phi_{i, E^{+}} \phi_{j, E^{+}}, \quad \mathbf{I}_{i, j}^{+/+}=\int_{F}\left(\nabla \phi_{i, E^{+}} \cdot \mathbf{n}^{+}\right) \phi_{j, E^{+}},
$$

for $i, j=1, \ldots, N_{p_{E^{+}}}$. We next recall how to efficiently compute terms of the form

$$
\begin{aligned}
& \mathbf{S}_{i, j}^{+/+}=\int_{F}\left(\phi_{i, E^{+}} \mathbf{n}^{+}\right) \cdot\left(\phi_{j, E^{+}} \mathbf{n}^{+}\right)=\int_{F} \phi_{i, E^{+}} \phi_{j, E^{+}}, \\
& \mathbf{S}_{i, j}^{+/-}=\int_{F}\left(\phi_{i, E^{+}} \mathbf{n}^{+}\right) \cdot\left(\phi_{j, E^{-}} \mathbf{n}^{-}\right)=-\int_{F} \phi_{i, E^{+}} \phi_{j, E^{-}},
\end{aligned}
$$

and refer to [22] for further details and discussion on the efficient computation of the terms $\mathbf{I}_{i, j}^{ \pm / \mp}$. Reasoning as before, we obtain

$$
\begin{aligned}
& \mathbf{S}_{i, j}^{+/+}=\sum_{k=0}^{i_{1}+j_{1}} \sum_{l=0}^{i_{2}+j_{2}} \mathscr{C}_{i_{1}, j_{1}, k} \mathscr{C}_{i_{2}, j_{2}, l} \mathscr{J}_{F^{+}} \int_{\hat{F}^{+}} \hat{x}^{k} \hat{y}^{l}, \\
& \mathbf{S}_{i, j}^{+/-}=-\sum_{k=0}^{i_{1}+j_{1} i_{2}+j_{2}} \sum_{l=0} \tilde{\mathscr{X}}_{i_{1}, j_{1}, k} \tilde{\mathscr{Y}}_{i_{2}, j_{2}, l} \mathscr{J}_{F^{+}} \int_{\hat{F}^{+}} \hat{x}^{k} \hat{y}^{l},
\end{aligned}
$$

where $\mathscr{J}_{F^{+}}$is defined as $\mathscr{J}_{F^{+}}=\left\|\mathbf{J}_{E^{+}}^{-\top} \hat{\mathbf{n}}_{\hat{F}+}\right\|\left|\mathbf{J}_{E^{+}}\right|$and $\hat{\mathbf{n}}_{\hat{F}^{+}}$is the unit outward normal vector to $\hat{F}^{+}$. In (17), the coefficients $\mathscr{C}_{i, j, k}$ are defined as in (16), whereas $\tilde{\mathscr{X}}$ and $\tilde{\mathscr{Y}}$ are defined as

$$
\left.\begin{array}{l}
\tilde{\mathscr{X}}_{i, j, k}=\sum_{n+m=k}\left(C_{i, n} \tilde{X}_{j, m}\right) \\
\tilde{\mathscr{Y}}_{i, j, k}=\sum_{n+m=k}\left(C_{i, n} \tilde{Y}_{j, m}\right)
\end{array}\right\} \quad \text { for } 0 \leq i \leq p_{E^{+}}, 0 \leq j \leq p_{E^{-}}, 0 \leq k \leq i+j .
$$

Here, as before, $C_{i, n}$ are the coefficients of the homogeneous function expansion of the Legendre polynomials in $(-1,1)$, while $\tilde{X}_{j, m}$ and $\tilde{Y}_{j, m}$ are defined by

$$
\left.\begin{array}{rl}
\tilde{X}_{j, m} & =\sum_{r=m}^{j} C_{j, r}\left(\begin{array}{c}
r \\
m
\end{array}\right)\left(\tilde{\mathbf{J}}_{1,1}\right)^{m}\left(\tilde{\mathbf{t}}_{1}\right)^{r-m} \\
\tilde{Y}_{j, m} & =\sum_{r=m}^{j} C_{j, r}\left(\begin{array}{c}
r \\
m
\end{array}\right)\left(\tilde{\mathbf{J}}_{2,2}\right)^{m}\left(\tilde{\mathbf{t}}_{2}\right)^{r-m}
\end{array}\right\} \quad \text { for } 0 \leq m \leq p_{E^{-}}, m \leq j \leq p_{E^{-}} .
$$


Fig. 6 Example of polygonal elements $E^{ \pm} \in \mathscr{T}_{h}$, together with the bounded boxes $B_{E^{ \pm}}$, and the local maps $\mathbf{F}_{E^{ \pm}}$: $\hat{E} \rightarrow E^{ \pm}$for the common face $F \subset E^{ \pm}$. Figure taken from [22].

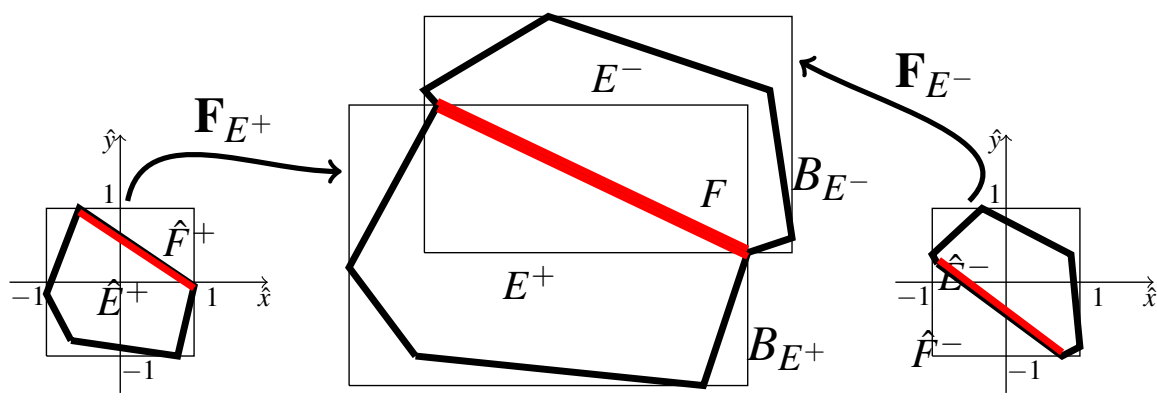

Finally, in the definition above $\tilde{\mathbf{t}}_{1}$ and $\tilde{\mathbf{t}}_{2}$ are the two components of the vector $\tilde{\mathbf{t}}$ of the composite map $\tilde{\mathbf{F}}(\hat{\mathbf{x}})=$ $\mathbf{F}_{E^{-}}^{-1}\left(\mathbf{F}_{E^{+}}(\hat{\mathbf{x}})\right)$, cf. Figure 6, defined as

$$
\tilde{\mathbf{F}}(\hat{\mathbf{x}})=\mathbf{J}_{E^{-}}^{-1}\left(\mathbf{J}_{E^{+}} \hat{\mathbf{x}}+\mathbf{t}_{E^{+}}\right)-\mathbf{J}_{E^{-}}^{-1} \mathbf{t}_{E^{-}}=\underbrace{\mathbf{J}_{E^{-}}^{-1} \mathbf{J}_{E^{+}}}_{\tilde{\mathbf{J}}} \hat{\mathbf{x}}+\underbrace{\mathbf{J}_{E^{-}}^{-1}\left(\mathbf{t}_{E^{+}}-\mathbf{t}_{E^{-}}\right)}_{\tilde{\mathbf{t}}} .
$$

We conclude this section by observing that for the computation of the local forcing term

$$
\int_{E} f(\boldsymbol{x}) \phi_{i, E}(\boldsymbol{x}) \mathrm{d} \boldsymbol{x}, i=1, \ldots, N_{p_{E}}
$$

the quadrature free method allows to exactly evaluate (18) when $f$ is a polynomial function. If $f$ is a general function, an explicit polynomial approximation of $f$ is required.

\subsection{Numerical results}

The aim of this section is to present some numerical computations to assess the practical performance of the quadrature free algorithm.

\subsubsection{Example 1: Integration of bivariate polynomials over a given polygon}

We first present some numerical results in order to test the performance of the method proposed in Algorithm 1 for the integration of bivariate polynomials over a given polygon $\mathscr{P} \subset \mathbb{R}^{2}$ based on employing the recursive algorithm described in Section 3.1, i.e., $\int_{\mathscr{P}} x^{k} y^{l}=\mathscr{I}(2, \mathscr{P}, k, l)$.

For the sake of comparison, we also present the analogous computations carried out based on employing the subtessellation technique. In this case, the domain of integration $\mathscr{P}$ is firstly decomposed into triangles; then on each subtriangle we employ a tensor product Gauss quadrature rule consisting of $\mathscr{N}^{2}$ nodes and weights, which is defined based

Fig. 7 Example 1 (Section 3.3.1). Domains of integration $\mathscr{P}$ : triangle $\left(\mathscr{P}_{a}\right.$, left) and an irregular polygon with 15 faces $\left(\mathscr{P}_{b}\right.$, right).

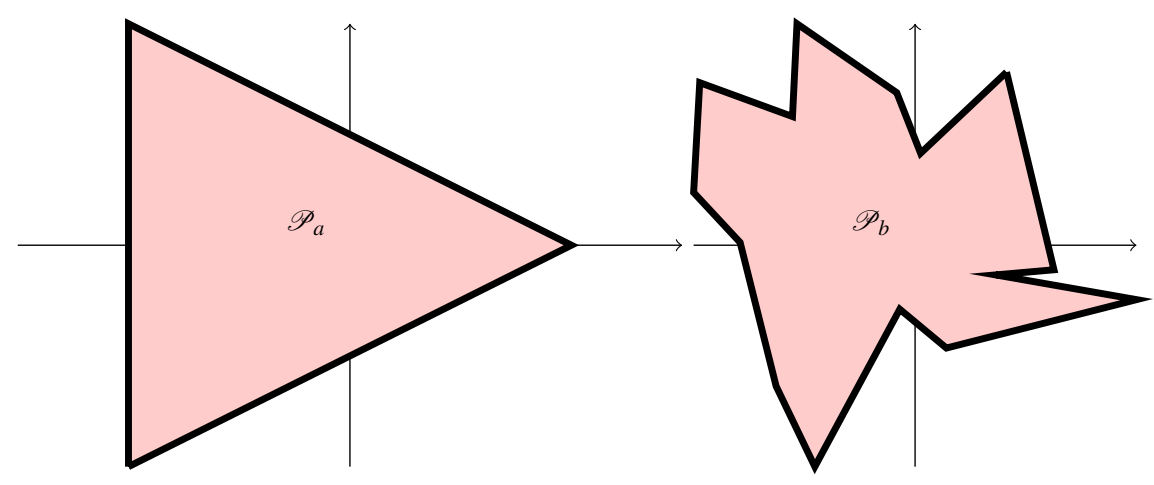


Table 2 Example 1 (Section 3.3.1). Vertex coordinates of polygons $\mathscr{P}_{a}$ and $\mathscr{P}_{b}$ of Figure 7.

\begin{tabular}{c|c|c} 
& vertex & $(x, y)$-coordinates \\
\hline & 1 & $(-1.000000000000000,-1.000000000000000)$ \\
$\mathscr{P}_{a}$ & 2 & $(1.000000000000000,0.000000000000000)$ \\
& 3 & $(-1.000000000000000,1.000000000000000)$ \\
\hline & 1 & $(0.413058522141662,0.781696234443715)$ \\
& 2 & $(0.024879797655533,0.415324992429711)$ \\
& 3 & $(-0.082799691823524,0.688810136531751)$ \\
& 4 & $(-0.533191422779328,1.000000000000000)$ \\
& 5 & $(-0.553573605852999,0.580958514816226)$ \\
& 6 & $(-0.972432940212767,0.734117068746903)$ \\
& 7 & $(-1.000000000000000,0.238078507228890)$ \\
$\mathscr{P}_{b}$ & 8 & $(-0.789986179147920,0.012425068086110)$ \\
& 9 & $(-0.627452906935866,-0.636532897516109)$ \\
& 10 & $(-0.452662174765764,-1.000000000000000)$ \\
& 11 & $(-0.069106265580153,-0.289054989277619)$ \\
& 12 & $(0.141448047807069,-0.464417038155806)$ \\
& 13 & $(1.000000000000000,-0.245698820584615)$ \\
& 14 & $(0.363704451489016,-0.134079689960635)$ \\
& 15 & $(0.627086024018283,-0.110940423607648)$ \\
\hline
\end{tabular}

on application of the Duffy transformation. In order to guarantee the exact integration of $x^{k} y^{l}$, we select $\mathscr{N}=\left\lceil\frac{k+l}{2}\right\rceil+1$. In order to compare both approaches, we integrate bivariate polynomials of different degrees on the triangle and the irregular polygon depicted in Figure 7; see Table 2 for the list of vertex coordinates for both domains. In Figures 8 and 9 we compare the CPU time (in seconds) taken to evaluate the underlying integral (on $\mathscr{P}_{a}$ and $\mathscr{P}_{b}$, respectively) up to machine precision, using the quadrature free algorithm and the sub-tessellation method. We remark that the times for the quadrature free algorithm include the computation of $b_{i}, \mathbf{n}_{i}$, and $d_{i j}, j=1, \ldots, m_{i}, i=1, \ldots, m$. The times for sub-tessellation method include the one-time computation of the $\mathscr{N}^{2}$ nodes and weights on the reference triangle, the time required for sub-tessellation, as well as the time needed for numerical integration on each sub-triangle. From the results reported in Figures 8 and 9 it is clear that the quadrature free algorithm outperforms sub-tessellation; indeed,

Fig. 8 Example 1 (Section 3.3.1). CPU times as a function of the integrand. Integration domain $\mathscr{P}_{a}$ of Figure 7.

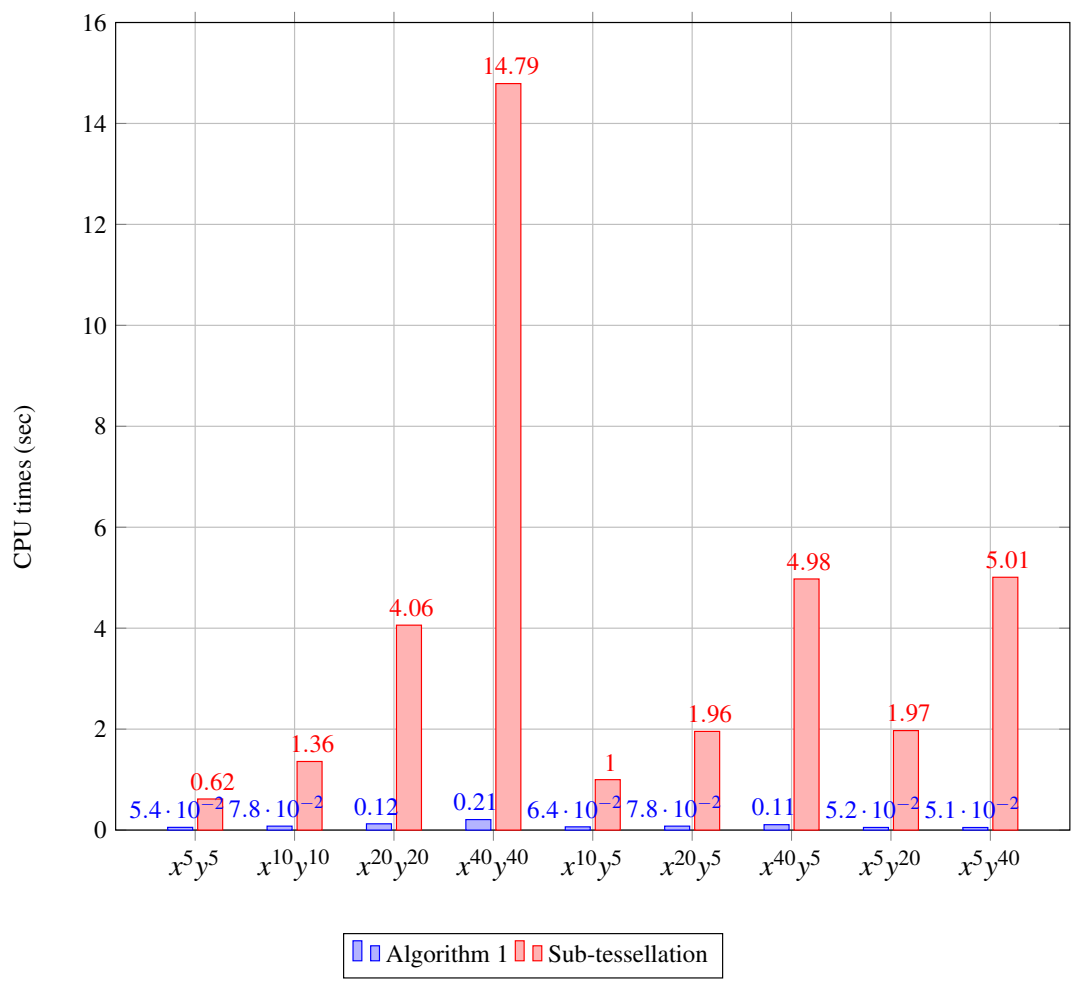


Fig. 9 Example 1 (Section 3.3.1). CPU times as a function of the integrand. Integration domain $\mathscr{P}_{b}$ of Figure 7.

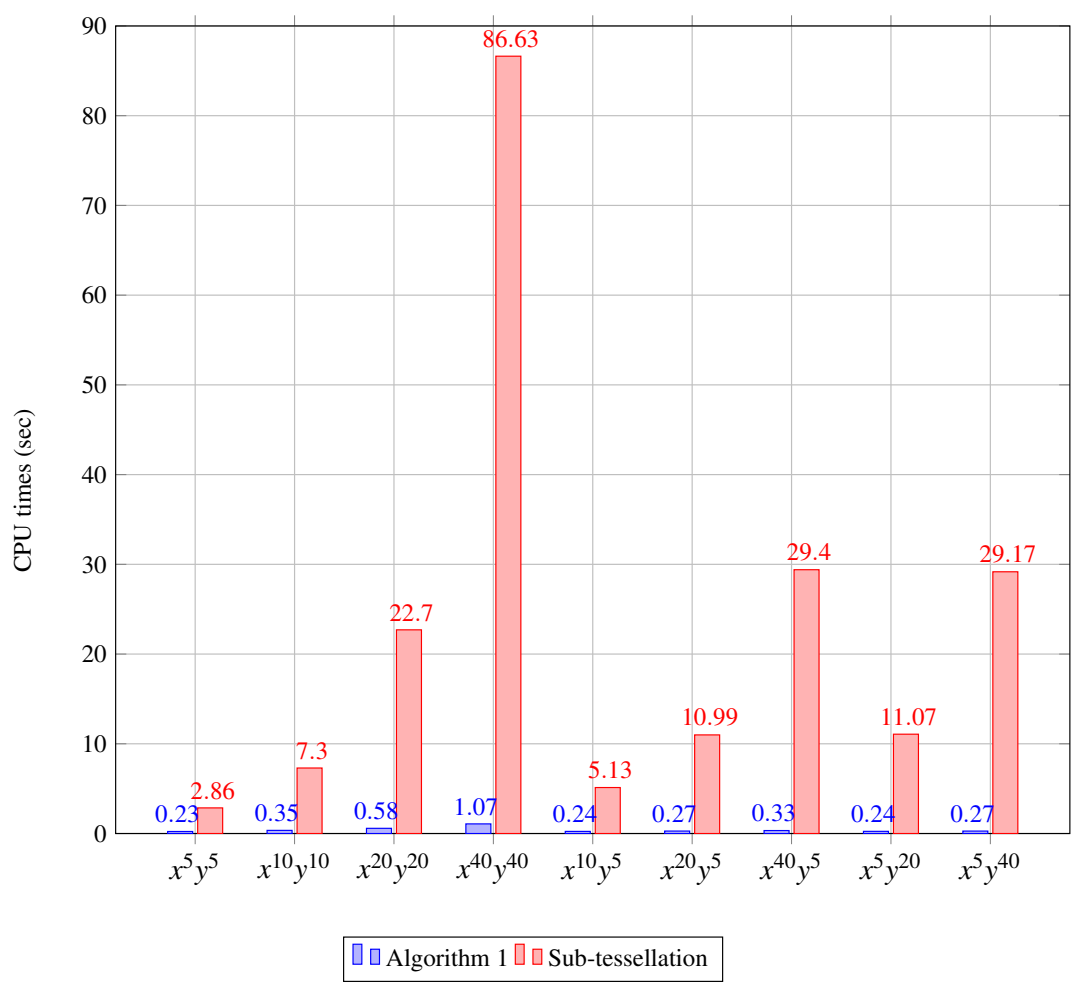

for both domains of integration, we observe an improvement in the CPU-time required to evaluate the underlying integral of between one- to two-orders of magnitude when the former approach is employed. Moreover, even when the integration domain consists of a triangle $\left(\mathscr{P}_{a}\right)$, the quadrature free algorithm still outperforms classical quadrature rules, even though in this case no sub-tessellation is undertaken.

\subsubsection{Example 2: Computation of the PolyDG stiffness and mass matrices in three-dimensions}

We now compare the performance of the quadrature free algorithm and the sub-tessellation method when employed to assemble the stiffness and mass matrices for the PolyDG approximation of a second-order elliptic diffusion-reaction problem in three-dimensions. Here, the polyhedral grids have been obtained by agglomeration starting from a partition $\Omega$ consisting of hexahedral elements. The agglomeration is performed based on employing the METIS library for graph partitioning, cf., for example, $[108,109]$ so that each polyhedral element is typically non-convex. In Figure 10 we show three typical examples of polyhedral elements generated from the agglomeration process. We now compare the CPU time required by the quadrature free method with the quadrature integration/sub-tessellation approach to assemble the volume and mass matrices, denoted by $\mathbf{V}$ and $\mathbf{M}$, respectively, as well for the computation of the interface matrices $\mathbf{S}$ and $\mathbf{I}$; cf. Section 3.2. We point out that, to assemble the volume and mass matrices based on employing the sub-tessellation algorithm, we exploit the fact that the polyhedral mesh is obtained by agglomeration of hexahedral

Fig. 10 Example 2 (Section 3.3.2). Typical agglomerated element shapes.
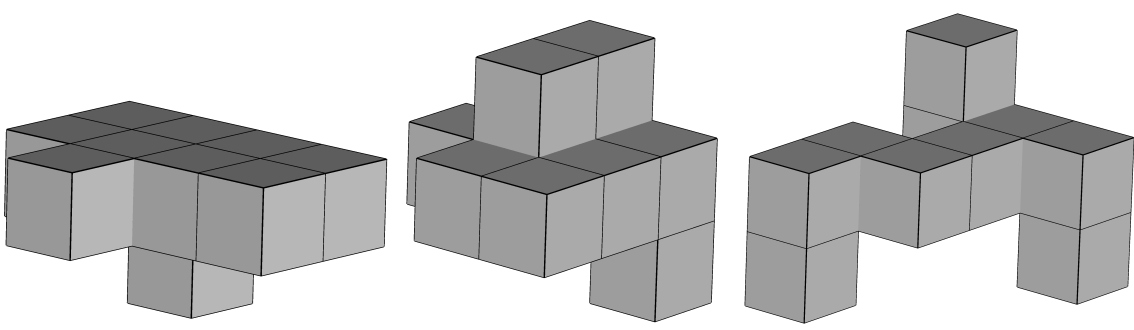

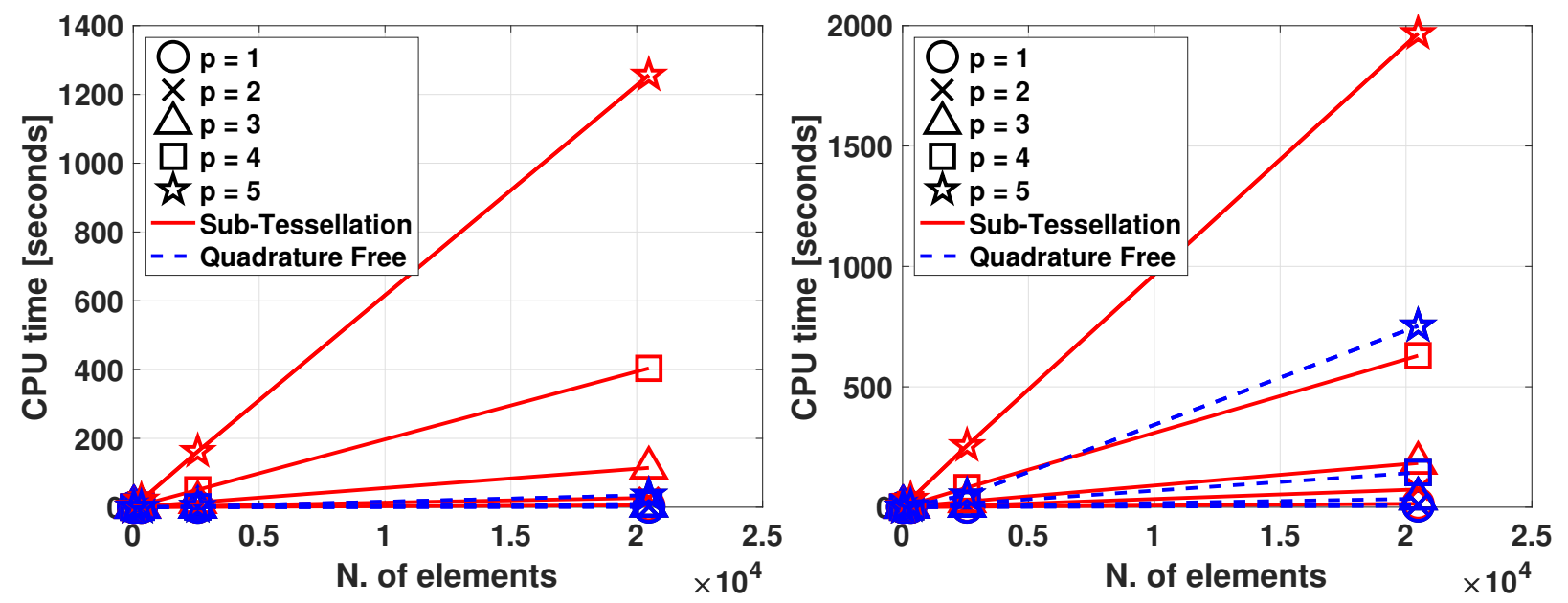

Fig. 11 Example 2 (Section 3.3.2). Comparison of the CPU time needed to assemble the volume matrices $\mathbf{M}$ and $\mathbf{V}$ (left) and the interface matrices $\mathbf{S}$ and $\mathbf{I}$ (right) for a three-dimensional problem by using the proposed quadrature free method and the classical sub-tessellation method. Each line is obtained by fixing the polynomial approximation degree $p \in\{1,2,3,4,5\}$ and measuring the CPU time by varying the number of elements $\left(N_{e}\right)$ of the underlying mesh.
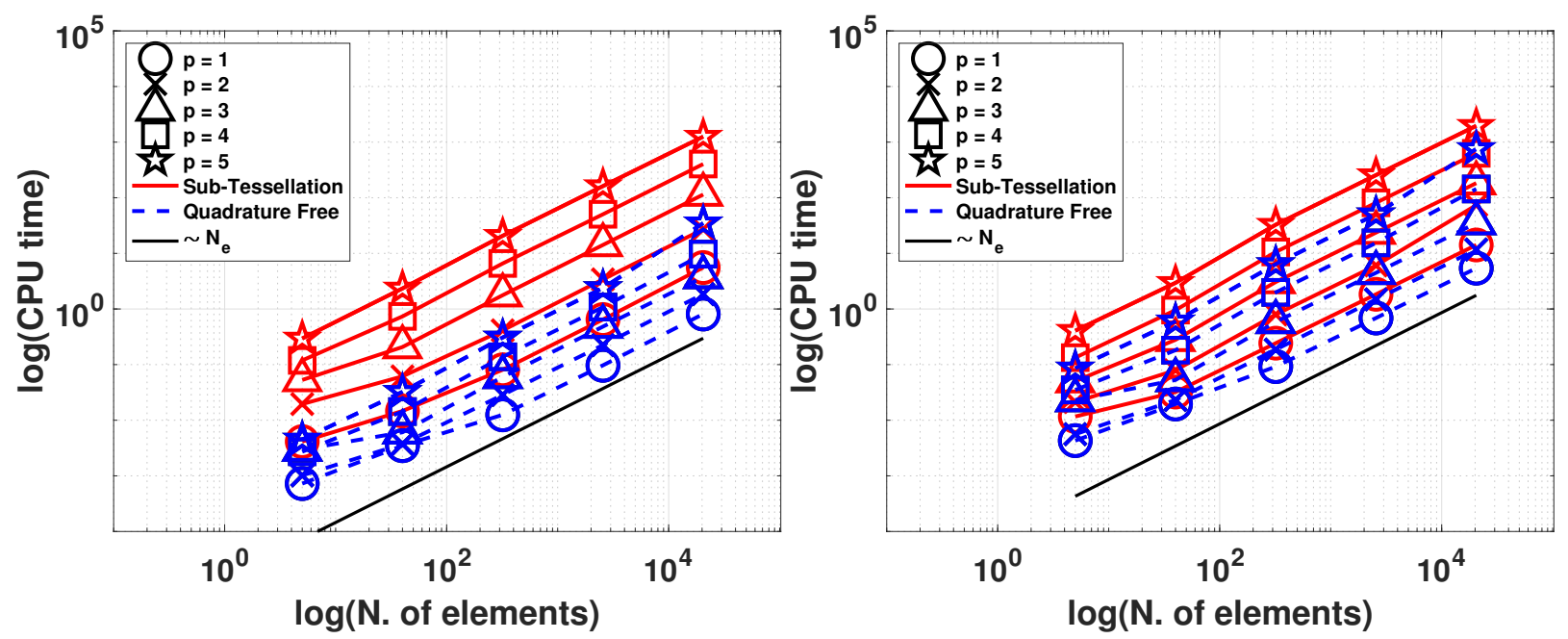

Fig. 12 Example 2 (Section 3.3.2).. Comparison of the CPU time needed to assemble the volume matrices $\mathbf{M}$ and $\mathbf{V}$ (left) and the interface matrices $\mathbf{S}$ and $\mathbf{I}$ (right) for a three-dimensional problem by using the proposed quadrature free method and the classical sub-tessellation method. Each line is obtained by fixing the polynomial approximation degree $p \in\{1,2,3,4,5\}$ and measuring the CPU time by varying the number of elements $\left(N_{e}\right)$ of the underlying mesh.

elements, so that the sub-tessellation into hexahedra of each polyhedral mesh element is already given. In Figure 11 (left) we report the CPU time needed for the computation of the volume matrices $\mathbf{V}$ and $\mathbf{M}$, for a set of agglomerated polyhedral grids where we fix the polynomial approximation degree $p \in\{1,2,3,4,5\}$ and we vary the number of elements $N_{e} \in\{5,40,320,2560,20480\}$; in all cases the agglomerated elements are formed from approximately 10 (fine) hexahedral elements. The analogous results obtained based on computing the interface matrices $\mathbf{S}$ and $\mathbf{I}$ (right) are shown in Figure 11 (right); furthermore, these timings are compared on a log-log plot in Figure 12. From the computations shown in Figures 11 and 12, we clearly observe that the quadrature free method substantially outperforms the sub-tessellation quadrature approach, both for the computation of the volume and the face integrals. We refer to [22] for additional numerical computations, where the issue of computational complexity is also addressed. 


\section{PolyDG methods for seismic wave propagation}

In this section we present an overview of high-order PolyDG methods for the approximate solution of wave propagation problems modeled by the elastodynamics equations on computational meshes consisting of polytopic elements. In particular, we discuss the model problem, analyze the well-posedness of the semidiscrete formulation and derive an $h p$-version a priori error bound. The theoretical estimates are then validated through two-dimensional numerical computations carried out on both benchmark, as well as real test cases. The dispersion analysis, in two-dimensions, is not reported here, for the sake of brevity, and can be found in [26], where it has been shown that polygonal meshes behave similarly to classical simplicial/quadrilateral grids in terms of dispersion errors. For the sake of brevity, we focus here on the elastodynamics equation; more sophisticated model problems can be successfully treated as well, for example, see [11, 10] and [28], respectively, for elasto-acoustic coupling and non-linear sound waves phenomena.

\subsection{Model problem and its PolyDG semidiscretization}

We consider an elastic body occupying an open, bounded polyhedral domain $\Omega \subset \mathbb{R}^{d}, d=2,3$, and denote by $\mathbf{n}$ the outward normal unit vector to its boundary. The boundary $\partial \Omega$ is assumed to be composed of two disjoint portions $\Gamma_{D} \neq \emptyset$ and $\Gamma_{N}$, i.e., $\Gamma_{D} \cap \Gamma_{N}=\emptyset$. For a final observation time $T>0$, let $\mathbf{u}: \Omega \times[0, T] \rightarrow \mathbb{R}^{d}$ be the displacement vector. The equations of the initial/boundary-value problem of (linear) elastodynamics are given by

$$
\left\{\begin{aligned}
\rho \ddot{\mathbf{u}}-\nabla \cdot \sigma & =\mathbf{f}, & & \text { in } \Omega \times(0, T], \\
\mathbf{u} & =\mathbf{0}, & & \text { on } \Gamma_{D} \times(0, T], \\
\sigma \mathbf{n} & =\mathbf{0}, & & \text { on } \Gamma_{N} \times(0, T], \\
\mathbf{u} & =\mathbf{u}_{0}, & & \text { in } \Omega \times\{0\}, \\
\dot{\mathbf{u}} & =\mathbf{u}_{1}, & & \text { in } \Omega \times\{0\} .
\end{aligned}\right.
$$

Here, $\mathbf{f} \in L^{2}\left((0, T] ; L^{2}(\Omega)\right)$ is the (given) external load and $\mathbf{u}_{0} \in H_{0, \Gamma_{D}}^{1}(\Omega)$ and $\mathbf{u}_{1} \in L^{2}(\Omega)$ are (given) initial data, where $H_{0, \Gamma_{D}}^{1}(\Omega)$ denotes the space of vector-valued functions in $H^{1}(\Omega)$ whose trace vanishes on $\Gamma_{D}$. Finally, $\rho \in$ $L^{\infty}(\Omega)$ is the medium density. As constitutive law for the stress tensor $\sigma: \Omega \times[0, T] \rightarrow \mathbb{S}, \mathbb{S}$ being the space of $d \times d$ symmetric real-valued matrices, $d=2,3$, we assume the generalized Hooke's law, i.e., $\sigma(\mathbf{u})=\mathscr{D} \varepsilon(\mathbf{u})$, where the fourth order stiffness tensor $\mathscr{D}: \mathbb{S} \rightarrow \mathbb{S}$ is defined as $\mathscr{D} \boldsymbol{\tau}=2 \mu \boldsymbol{\tau}+\lambda \operatorname{tr}(\boldsymbol{\tau}) \mathbf{I}$ for any $\boldsymbol{\tau} \in \mathbb{S}$, and $\varepsilon(\mathbf{u})$ is the symmetric gradient of $\mathbf{u}$, i.e., $\varepsilon(\mathbf{u})=\frac{1}{2}\left(\nabla \mathbf{u}+\nabla \mathbf{u}^{\top}\right)$. Here, $\mathbf{I}$ is the identity tensor, $\operatorname{tr}(\cdot)$ represents the trace operator, and $\lambda, \mu \in L^{\infty}(\Omega)$ are the first and the second Lamé parameters, respectively. We assume that $\mathscr{D}$ is symmetric, positive definite and uniformly bounded over $\Omega$. We recall that the compressional (P) and shear (S) wave velocities can be obtained through the relations $c_{P}=\sqrt{(\lambda+2 \mu) / \rho}$ and $c_{S}=\sqrt{\mu / \rho}$, respectively. The weak formulation of problem (19) reads as follows: for all $t \in(0, T]$ find $\mathbf{u}=\mathbf{u}(t) \in \mathbf{H}_{0, \Gamma_{D}}^{1}(\Omega)$ such that:

$$
\left\{\begin{array}{l}
\int_{\Omega} \rho \ddot{\mathbf{u}} \cdot \mathbf{v}+\int_{\Omega} \mathscr{D} \varepsilon(\mathbf{u}): \varepsilon(\mathbf{v})=\int_{\Omega} \mathbf{f} \cdot \mathbf{v} \quad \forall \mathbf{v} \in \mathbf{H}_{0, \Gamma_{D}}^{1}(\Omega), \\
\mathbf{u}(\cdot, 0)=\mathbf{u}_{0}, \quad \dot{\mathbf{u}}(\cdot, 0)=\mathbf{u}_{1} .
\end{array}\right.
$$

Problem (20) is well posed and its unique solution $\mathbf{u} \in C\left((0, T] ; \mathbf{H}_{0, \Gamma_{D}}^{1}(\Omega)\right) \cap C^{1}\left((0, T] ; L^{2}(\Omega)\right)$, see [130, Theorem 8-3.1].

Based on employing the notation of Section 2, we introduce the PolyDG semidiscretization of problem (20): for all $t \in(0, T]$, find $\mathbf{u}_{h}=\mathbf{u}_{h}(t) \in W_{h}^{D G}$ such that

$$
\int_{\Omega} \rho \ddot{\mathbf{u}}_{h} \cdot \mathbf{v}+\mathscr{B}\left(\mathbf{u}_{h}, \mathbf{v}\right)=\int_{\Omega} \mathbf{f} \cdot \mathbf{v} \quad \forall \mathbf{v} \in W_{h}^{D G},
$$

supplemented with the initial conditions $\mathbf{u}_{h}(0)=\mathbf{u}_{h}^{0}$ and $\dot{\mathbf{u}}_{h}(0)=\mathbf{u}_{h}^{1}$, where $\mathbf{u}_{h}^{0}, \mathbf{u}_{h}^{1} \in W_{h}^{D G}$ are suitable approximations of $\mathbf{u}_{0}$ and $\mathbf{u}_{1}$, respectively. Here, we also assume that $\mathscr{D}$ and $\rho$ are element-wise constant over the mesh $\mathscr{T}_{h}$. The 
bilinear form $\mathscr{B}(\cdot, \cdot): W_{h}^{D G} \times W_{h}^{D G} \rightarrow \mathbb{R}$ is defined as

$$
\mathscr{B}(\mathbf{u}, \mathbf{v})=\int_{\Omega} \sigma(\mathbf{u}): \varepsilon(\mathbf{v})+\int_{\Omega} \sigma(\mathbf{u}): \mathscr{R}\left(\llbracket[\mathbf{v} \rrbracket)+\int_{\Omega} \mathscr{R}\left(\llbracket[\mathbf{u} \rrbracket): \sigma(\mathbf{v})+\int_{\mathscr{F}_{h} I_{\mathscr{F}} D} \eta \llbracket[\mathbf{u} \rrbracket: \llbracket[\mathbf{v} \rrbracket \rrbracket\right.\right.
$$

for all $\mathbf{u}, \mathbf{v} \in W_{h}^{D G}$. Here, $\mathscr{R}(\cdot): L^{1}\left(\mathscr{F}_{h}^{I} \cup \mathscr{F}_{h}^{D}\right) \rightarrow \mathscr{W}_{h}^{D G}$ is the lifting operator of the traces of $d \times d$ symmetric tensors defined as

$$
\int_{\Omega} \mathscr{R}\left(\llbracket[\mathbf{w} \rrbracket \rrbracket): \sigma(\mathbf{v})=-\int_{\mathscr{F}{ }_{h}^{I} \cup \mathscr{F}{ }_{h}} \llbracket\left[\mathbf{w} \rrbracket:\{\sigma(\mathbf{v})\} \quad \forall \mathbf{v} \in W_{h}^{D G} .\right.\right.
$$

The penalization function $\eta: \mathscr{F}_{h} \rightarrow \mathbb{R}_{+}$in (22) is defined face-wise as

$$
\eta=\sigma_{0} \overline{\mathscr{D}}_{E} \begin{cases}\max _{E \in\left\{E_{1}, E_{2}\right\}}\left(\frac{p_{E}^{2}}{h_{E}}\right), & F \in \mathscr{F}_{h}^{I}, F \subset \partial E_{1} \cap \partial E_{2}, \\ \frac{p_{E}^{2}}{h_{E}}, & F \in \mathscr{F}_{h}^{D}, F \subset \partial E \cap \Gamma_{D} .\end{cases}
$$

where $\overline{\mathscr{D}}_{E}=\left|\left(\left.\mathscr{D}\right|_{E}\right)^{1 / 2}\right|_{2}^{2}$ for any $E \in \mathscr{T}_{h}$ (here $|\cdot|_{2}$ is the operator norm induced by the $l_{2}$-norm on $\mathbb{R}^{n}$, where $n$ denotes the dimension of the space of symmetric second-order tensors, i.e., $n=3$ if $d=2, n=6$ if $d=3$ ), and $\sigma_{0}$ is a positive parameter at our disposal.

\subsection{Well-posedness, stability and error analysis of the semidiscrete formulation}

In this section we prove stability and error estimates for the PolyDG semidiscretization defined in (21). To this end, we define the space $\widetilde{W}_{h}^{D G}=W_{h}^{D G} \oplus H_{0, \Gamma_{D}}^{1}(\Omega)$ endowed with the following DG norm

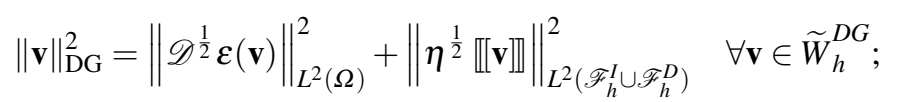

here, we have used the compact notation $\|\cdot\|_{L^{2}\left(\mathscr{F}{ }_{h}^{I} \cup \mathscr{F} h\right)}^{2}=\sum_{F \in \mathscr{F} I_{h} \cup \mathscr{F} D}\|\cdot\|_{L^{2}(F)}^{2}$. From the preliminary results of Section 2 we immediately have the following estimates; we refer to [26] for more details.

Lemma 3. Assume that $\mathscr{T}_{h}$ satisfies Assumption 2. Then, for any $\boldsymbol{w} \in W_{h}^{D G}$ we have that

$$
\left\|\eta^{-1 / 2}\{\boldsymbol{w}\}\right\|_{L^{2}\left(\mathscr{F}{ }_{h}^{I} \cup \mathscr{F} h\right)}^{2} \lesssim \frac{1}{\sigma_{0}}\|\boldsymbol{w}\|_{L^{2}(\Omega)},
$$

where the hidden constant is independent of $p_{E},|E|$, and $\boldsymbol{w}$, and where $\sigma_{0}$ is the constant appearing in the definition of the penalty function, $c f$. (24).

Lemma 4. Assume that $\mathscr{T}_{h}$ satisfies Assumption 2. For any $\boldsymbol{v} \in \widetilde{W}_{h}^{D G}$ we have that

$$
\| \mathscr{R}\left(\llbracket [ \boldsymbol { v } \rrbracket ) \| _ { L ^ { 2 } ( \Omega ) } ^ { 2 } \lesssim \frac { 1 } { \sigma _ { 0 } } \| \eta ^ { \frac { 1 } { 2 } } \llbracket \left[\boldsymbol{v} \rrbracket \|_{L^{2}\left(\mathscr{F}{ }_{h} \cup \mathscr{F} h\right)}^{2},\right.\right.
$$

where $\sigma_{0}$ is the constant appearing in the definition of the penalty function, $c f$. (24).

Proof. The proof follows by observing that if $\mathbf{v} \in H_{0, \Gamma_{D}}^{1}(\Omega)$, then $\llbracket\left[\mathbf{v} \rrbracket=0\right.$ and the estimate is trivial. If $\mathbf{v} \in W_{h}^{D G}$, by using the definition of the lifting operator (23) together with Lemma 3 the result follows immediately.

Based on employing the above results and standard DG arguments, the well-posedness of the PolyDG formulation (21) can be established.

Lemma 5. Assume that $\mathscr{T}_{h}$ satisfies Assumption 2, and that the constant $\sigma_{0}$ appearing in the definition (24) of the penalization function is chosen sufficiently large. Then, 


$$
\mathscr{B}(\boldsymbol{v}, \boldsymbol{v}) \gtrsim\|\boldsymbol{v}\|_{D G}^{2}, \quad \mathscr{B}(\boldsymbol{v}, \boldsymbol{w}) \lesssim\|\boldsymbol{v}\|_{D G}\|\boldsymbol{w}\|_{D G} \quad \forall \boldsymbol{v}, \boldsymbol{w} \in \widetilde{W}_{h}^{D G} .
$$

We next provide a stability result of the semidiscrete PolyDG formulation (21) in the following energy norm

$$
\left\|\mathbf{u}_{h}(t)\right\|_{\mathrm{E}}^{2}=\left\|\rho^{\frac{1}{2}} \dot{\mathbf{u}}_{h}(t)\right\|_{L^{2}(\Omega)}^{2}+\left\|\mathbf{u}_{h}(t)\right\|_{\mathrm{DG}}^{2} \quad \forall t \in(0, T] .
$$

Proposition 2. Let $\mathbf{f} \in L^{2}\left((0, T] ; \boldsymbol{L}^{2}(\Omega)\right)$ and $\mathbf{u}_{h} \in \mathscr{C}^{2}\left((0, T] ; W_{h}^{D G}\right)$ be the approximate solution of (21) obtained with the stability constant $\sigma_{0}$ defined in (24) chosen sufficiently large. Then,

$$
\left\|\mathbf{u}_{h}(t)\right\|_{E} \lesssim\left\|\mathbf{u}_{h}^{0}\right\|_{E}+\int_{0}^{t}\|\mathbf{f}(\tau)\|_{L^{2}(\Omega)}, \quad 0<t \leq T .
$$

Proof. Selecting $\mathbf{v}=\dot{\mathbf{u}}_{h} \in W_{h}^{D G}$ in (21), integrating in time between 0 and $t$, employing Lemma 3 together with the arithmetic-geometric inequality, and choosing $\sigma_{0}$ large enough, we get

$$
\left\|\mathbf{u}_{h}\right\|_{\mathrm{E}}^{2}+2 \int_{\Omega} \mathscr{R}\left(\llbracket\left[\mathbf{u}_{h} \rrbracket\right): \sigma\left(\mathbf{u}_{h}\right) \gtrsim\left\|\mathbf{u}_{h}\right\|_{\mathrm{E}}^{2} .\right.
$$

Moreover, from Lemma 4 it also follows that

$$
2 \mid \int_{\Omega} \mathscr{R}\left(\llbracket\left[\mathbf{u}_{h} \rrbracket \rrbracket^{0}\right): \sigma\left(\mathbf{u}_{h}^{0}\right) \mid \lesssim \frac{1}{\sqrt{\sigma_{0}}}\left\|\eta^{\frac{1}{2}} \llbracket \llbracket \mathbf{u}_{h}^{0} \rrbracket\right\|_{L^{2}\left(\mathscr{F}_{h}^{I} \cup \mathscr{F}_{h}^{D}\right)}\left\|\sigma\left(\mathbf{u}_{h}^{0}\right)\right\|_{L^{2}(\Omega)} \lesssim \frac{1}{\sqrt{\sigma_{0}}}\left\|\mathbf{u}_{h}^{0}\right\|_{\mathrm{E}}^{2} .\right.
$$

Therefore, substituting these inequalities, and applying the Cauchy-Schwarz inequality yields

$$
\left\|\mathbf{u}_{h}\right\|_{\mathrm{E}}^{2} \lesssim\left\|\mathbf{u}_{h}^{0}\right\|_{\mathrm{E}}^{2}+2 \int_{0}^{t}\left\|\mathbf{u}_{h}\right\|_{\mathrm{E}}\|\mathbf{f}\|_{L^{2}(\Omega)} .
$$

The statement of the proposition now follows by employing Gronwall's lemma [129].

Before providing $h p$-version error bounds, we observe that formulation (21) is not strongly-consistent, due to the presence of the lifting operator. It is easy to see that the error $\mathbf{u}-\mathbf{u}_{h}$ satisfies the following error equation

$$
\int_{\Omega} \rho\left(\ddot{\mathbf{u}}-\ddot{\mathbf{u}}_{h}\right) \cdot \mathbf{v}_{h}+\mathscr{B}\left(\mathbf{u}-\mathbf{u}_{h}, \mathbf{v}_{h}\right)+\mathscr{R}_{h}\left(\mathbf{u}-\mathbf{u}_{h}, \mathbf{v}_{h}\right)=0 \quad \forall \mathbf{v}_{h} \in W_{h}^{D G},
$$

where the residual $\mathscr{R}_{h}(\cdot, \cdot): \widetilde{W}_{h}^{D G} \times W_{h}^{D G} \rightarrow \mathbb{R}$ is defined by

$$
\mathscr{R}_{h}\left(\mathbf{w}, \mathbf{v}_{h}\right)=-\int_{\mathscr{F}_{h}^{I} \cup \mathscr{F} D}\{\sigma(\mathbf{w})\}: \llbracket \llbracket \mathbf{v}_{h} \rrbracket-\int_{\Omega} \sigma(\mathbf{w}): \mathscr{R}\left(\llbracket \mathbf{v}_{h} \rrbracket\right) \quad \forall \mathbf{w} \in \widetilde{W}_{h}^{D G} \forall \mathbf{v}_{h} \in W_{h}^{D G},
$$

and where we have used also that $\mathscr{R}_{h}\left(\mathbf{w}_{h}, \mathbf{v}_{h}\right)=0$ whenever $\mathbf{w}_{h} \in W_{h}^{D G}$, cf. (23).

In order to derive a priori error bounds for the semidiscrete scheme, we assume that Assumption 4 is satisfied; we define, component-wise, the extension operators $\mathscr{E}: \mathscr{H}^{s}(\Omega) \rightarrow \mathscr{H}^{s}\left(\mathbb{R}^{d \times d}\right), s \in \mathbb{N}_{0}$, as in Section ??, cf. also [136]; we employ the tensorial and vectorial counterpart of the approximation estimates outlined in Section ??, cf. also [63, 26], to obtain the following bound

$$
\|\mathbf{u}-\Pi \mathbf{u}\|_{\mathrm{E}}^{2} \lesssim \sum_{E \in \mathscr{T}_{h}} \frac{h_{E}^{2\left(s_{E}-1\right)}}{p_{E}^{2\left(r_{E}-3 / 2\right)}}\left(\|\mathscr{E} \mathbf{u}\|_{\mathbf{H}^{r} E\left(T_{E}\right)}^{2}+\frac{h_{E}^{2}}{p_{E}^{3}}\|\mathscr{E} \dot{\mathbf{u}}\|_{\mathbf{H}^{r} E\left(T_{E}\right)}^{2}\right),
$$

where $s_{E}=\min \left(p_{E}+1, r_{E}\right)$. The hidden constant depends on the material parameters and on the shape-regularity of $T_{E}$, but is independent of $q, h_{E}, p_{E}$ and the number of faces per element. Moreover, the global interpolant $\Pi$ is defined elementwise as $\left.\Pi \mathbf{u}\right|_{E}=\Pi_{E}^{p_{E}} \mathbf{u}$ for any $E \in \mathscr{T}_{h}$, where $\Pi_{E}^{p_{E}}$ is vector-valued counterpart of the interpolant defined in Lemma 2.

The last ingredient we need is the following bound on the residual; we refer to [26] for the proof. 
Lemma 6. For any $\boldsymbol{w} \in \widetilde{W}_{h}^{D G}$ and $\boldsymbol{v}_{h} \in W_{h}^{D G}$, the following bound holds

$$
\left|\mathscr{R}_{h}\left(\boldsymbol{w}, \boldsymbol{v}_{h}\right)\right| \lesssim\left(\sum_{E \in \mathscr{T}_{h}} \frac{h_{E}^{2\left(s_{E}-1\right)}}{p_{E}^{2\left(r_{E}-3 / 2\right)}}\|\mathscr{E} \boldsymbol{\sigma}(\boldsymbol{w})\|_{\mathscr{H}^{r_{E}}\left(T_{E}\right)}^{2}\right)^{1 / 2}\left\|\boldsymbol{v}_{h}\right\|_{D G},
$$

where $s_{E}=\min \left(p_{E}+1, r_{E}\right)$ for all $E \in \mathscr{T}_{h}$. The hidden constant depends on the material parameters and the shaperegularity of $T_{E}$, but is independent of $q, h_{E}, p_{E}$, and the number of element faces.

We can now state the main result of this section.

Theorem 1. Let Assumption 2 and Assumption 4 be satisfied. Moreover, assume that the analytical solution $\boldsymbol{u}$ of (19) is sufficiently regular. For any time $t \in[0, T]$, let $\mathbf{u}_{h} \in W_{h}^{D G}$ be the PolyDG solution of problem (21) obtained with a penalty parameter $\sigma_{0}$ appearing in (24) sufficiently large. Then, for any time $t \in(0, T]$ the following bound holds

$$
\begin{aligned}
\left\|\mathbf{u}-\mathbf{u}_{h}\right\|_{E}^{2} \lesssim & \sum_{E \in \mathscr{T}_{h}} \frac{h_{E}^{2\left(s_{E}-1\right)}}{p_{E}^{2\left(r_{E}-3 / 2\right)}}\left(\|\mathscr{E} \mathbf{u}\|_{H^{r} E\left(T_{E}\right)}^{2}+\frac{h_{E}^{2}}{p_{E}^{3}}\|\mathscr{E} \dot{\mathbf{u}}\|_{H^{r_{E}\left(T_{E}\right)}}^{2}+\|\mathscr{E} \sigma(\boldsymbol{u})\|_{\mathscr{H}^{r} E\left(T_{E}\right)}^{2}\right) \\
& +\frac{h_{E}^{2\left(s_{E}-1\right)}}{p_{E}^{2\left(r_{E}-3 / 2\right)}} \int_{0}^{t}\left(\|\mathscr{E} \dot{\mathbf{u}}\|_{H^{r} E\left(T_{E}\right)}^{2}+\frac{h_{E}^{2}}{p_{E}^{3}}\|\mathscr{E} \dot{\mathbf{u}}\|_{H^{r} E\left(T_{E}\right)}^{2}+\|\mathscr{E} \sigma(\dot{\boldsymbol{u}})\|_{\mathscr{H}^{r} E\left(T_{E}\right)}^{2}\right),
\end{aligned}
$$

with $s_{E}=\min \left(p_{E}+1, m_{k}\right)$ for all $E \in \mathscr{T}_{h}$. The hidden constants depends on the material parameters and the shaperegularity of $T_{E}$, but is independent of $q, h_{E}, p_{E}$ and the number of element faces.

Proof. We recall the main steps of the proof and refer to [26] for more details. Let $\Pi$ be defined as (27). We write the error as $\mathbf{u}-\mathbf{u}_{h}=\mathbf{e}_{h}-\mathbf{e}_{I}$ with $\mathbf{e}_{h}=\mathbf{u}_{h}-\Pi \mathbf{u}$ and $\mathbf{e}_{I}=\mathbf{u}-\Pi \mathbf{u}$, and rewrite the error equation (26) for $\mathbf{v}_{h}=\dot{\mathbf{e}}_{h}$, to obtain

$$
\int_{\Omega} \rho \ddot{\mathbf{e}}_{h} \cdot \dot{\mathbf{e}}_{h}+\mathscr{B}\left(\mathbf{e}_{h}, \dot{\mathbf{e}}_{h}\right)=\int_{\Omega} \rho \ddot{\mathbf{e}}_{I} \cdot \dot{\mathbf{e}}_{h}+\mathscr{B}\left(\mathbf{e}_{I}, \dot{\mathbf{e}}_{h}\right)+\mathscr{R}_{h}\left(\mathbf{e}_{I}, \dot{\mathbf{e}}_{h}\right),
$$

where we have also used that $\mathscr{R}_{h}\left(\mathbf{e}_{h}, \dot{\mathbf{e}}_{h}\right)=0$ since $\mathbf{e}_{h}, \dot{\mathbf{e}}_{h} \in W_{h}^{D G}$. Using the definition of the energy norm (25), integrating in time between 0 and $t$, and exploiting that $\mathbf{e}_{h}(0)=\mathbf{0}$, and reasoning as in the proof of Proposition 2 yields

$$
\left\|\mathbf{e}_{h}\right\|_{\mathrm{E}}^{2}+2 \int_{\Omega} \mathscr{R}\left(\llbracket\left[\mathbf{e}_{h} \rrbracket\right): \sigma\left(\mathbf{e}_{h}\right) \gtrsim\left\|\mathbf{e}_{h}\right\|_{\mathrm{E}}^{2}\right.
$$

provided the penalty parameter is chosen sufficiently large. Therefore, we get

$$
\begin{aligned}
\left\|\mathbf{e}_{h}\right\|_{\mathrm{E}}^{2} & \lesssim \int_{0}^{t} \int_{\Omega} \rho \ddot{\mathbf{e}}_{I} \cdot \dot{\mathbf{e}}_{h}+\int_{0}^{t} \mathscr{B}\left(\mathbf{e}_{I}, \dot{\mathbf{e}}_{h}\right)+\int_{0}^{t} \mathscr{R}_{h}\left(\mathbf{e}_{I}, \dot{\mathbf{e}}_{h}\right) \\
& =\int_{0}^{t} \int_{\Omega} \rho \ddot{\mathbf{e}}_{I} \cdot \dot{\mathbf{e}}_{h}+\mathscr{B}\left(\mathbf{e}_{I}, \mathbf{e}_{h}\right)-\int_{0}^{t} \mathscr{B}\left(\dot{\mathbf{e}}_{I}, \mathbf{e}_{h}\right)-\mathscr{R}_{h}\left(\mathbf{e}_{I}, \mathbf{e}_{h}\right)+\int_{0}^{t} \mathscr{R}_{h}\left(\dot{\mathbf{e}}_{I}, \mathbf{e}_{h}\right),
\end{aligned}
$$

where in the second step we have used integration by parts for the second and third term on the right hand side together with $\mathbf{e}_{h}(0)=\mathbf{0}$. Employing Jensen and Cauchy-Schwarz inequalities for first term on the right hand side, the fact that $\mathscr{R}_{h}\left(\mathbf{e}_{I}, \mathbf{e}_{h}\right)=\mathscr{R}_{h}\left(\mathbf{u}, \mathbf{e}_{h}\right)$, Lemma 5, the definition of the energy norm (25), and Lemma 6, we get

$$
\left\|\mathbf{e}_{h}\right\|_{\mathrm{E}}^{2} \lesssim\left\|\mathbf{e}_{I}\right\|_{\mathrm{E}}\left\|\mathbf{e}_{h}\right\|_{\mathrm{E}}+\int_{0}^{t}\left\|\dot{\mathbf{e}}_{I}\right\|_{\mathrm{E}}\left\|\mathbf{e}_{h}\right\|_{\mathrm{E}}+\mathscr{I}(\mathbf{u})\left\|\mathbf{e}_{h}\right\|_{\mathrm{E}}+\int_{0}^{t} \mathscr{I}(\dot{\mathbf{u}})\left\|\mathbf{e}_{h}\right\|_{\mathrm{E}},
$$

where

$$
\mathscr{I}(\mathbf{u})=\left(\sum_{E \in \mathscr{T}_{h}} \frac{h_{E}^{2\left(s_{E}-1\right)}}{p_{E}^{2\left(m_{E}-3 / 2\right)}}\|\mathscr{E} \sigma(\mathbf{u})\|_{\mathscr{H}^{m_{E}}\left(T_{E}\right)}^{2}\right)^{1 / 2},
$$

cf. Lemma 6. Applying the arithmetic-geometric inequality with $\delta>0$ we have 


$$
(1-\delta)\left\|\mathbf{e}_{h}\right\|_{\mathrm{E}}^{2} \lesssim \frac{1}{\delta}\left(\left\|\mathbf{e}_{I}\right\|_{\mathrm{E}}^{2}+\mathscr{I}^{2}(\mathbf{u})\right)+\int_{0}^{t}\left(\left\|\dot{\mathbf{e}}_{I}\right\|_{\mathrm{E}}+\mathscr{I}(\dot{\mathbf{u}})\right)\left\|\mathbf{e}_{h}\right\|_{\mathrm{E}}
$$

Choosing $\delta$ small enough and applying Gronwall's lemma [129] we get

$$
\left\|\mathbf{e}_{h}\right\|_{\mathrm{E}}^{2} \lesssim\left\|\mathbf{e}_{I}\right\|_{\mathrm{E}}^{2}+\mathscr{I}^{2}(\mathbf{u})+\int_{0}^{t}\left(\left\|\dot{\mathbf{e}}_{I}\right\|_{\mathrm{E}}^{2}+\mathscr{I}^{2}(\dot{\mathbf{u}})\right) .
$$

The proof is completed by employing (27) and the definition of $\mathscr{I}(\mathbf{u})$.

\subsection{Numerical results}

Before presenting some numerical experiments, we first discuss the algebraic formulation of the semidiscrete formulation and the time integration of the corresponding system of second-order ordinary differential equations. We suppose that $\Omega$ is partitioned into $N_{e l}$ disjoint polytopic elements $E_{r}, r=1, \ldots, N_{e l}$, and denote by $n_{p_{E}}=\operatorname{dim}\left(\mathbb{P}_{p_{E}}\right)$, and set $N_{d o f}=\sum_{r=1}^{N_{e l}} n_{p_{E}}$ to be the dimension of each component of a function in $W_{h}^{D G}$. We introduce a basis $\left\{\boldsymbol{\Phi}_{i}^{1}, \ldots, \boldsymbol{\Phi}_{i}^{d}\right\}_{i=1}^{N_{d o f}}$, $d=2,3$, for the finite element space $W_{h}^{D G}$. By expressing $\mathbf{u}_{h} \in W_{h}^{D G}$ as a linear combination of the basis functions, i.e.,

$$
\mathbf{u}_{h}(\mathbf{x}, t)=\sum_{s=1}^{d} \sum_{j=1}^{N_{\text {dof }}} \boldsymbol{\Phi}_{j}^{s}(\mathbf{x}) U_{j}^{s}(t)
$$

and writing equation (21) for any test function $\boldsymbol{\Phi}_{i}^{s}(\mathbf{x}) \in W_{h}^{D G}, s=1, \ldots, d$, we obtain the following system of second order differential equations

$$
M \ddot{\mathbf{U}}(t)+B \mathbf{U}(t)=\mathbf{F}(t) \quad \forall t \in(0, T),
$$

for the displacements $\mathbf{U}(t)=\left(\mathbf{U}^{1}(t), \ldots, \mathbf{U}^{d}(t)\right)^{T}$. Here, $\mathbf{F}=\left(\mathbf{F}^{1}(t), \ldots, \mathbf{F}^{d}(t)\right)^{T}$ represents the external applied load, $M$ and $B$ are the (symmetric and positive definite) mass and stiffness matrices, respectively. To integrate the system (28) in time we consider the explicit, second-order accurate, and conditionally stable leap-frog scheme: we subdivide the interval $(0, T]$ into $N_{T}$ equal subintervals of size $\Delta t=T / N_{T}$ and at every time level $t_{n}=n \Delta t$ we solve the system

$$
M \mathbf{U}\left(t_{n+1}\right)=\left[2 M-\Delta t^{2} B\right] \mathbf{U}\left(t_{n}\right)-M \mathbf{U}\left(t_{n-1}\right)+\Delta t^{2} \mathbf{F}\left(t_{n}\right), \quad \text { for } n=1, \ldots, N_{T},
$$

with

$$
M \mathbf{U}\left(t_{1}\right)=\left[M-\frac{\Delta t^{2}}{2} B\right] \mathbf{U}\left(t_{0}\right)-\Delta t M \dot{\mathbf{U}}\left(t_{0}\right)+\frac{\Delta t^{2}}{2} \mathbf{F}\left(t_{0}\right)
$$

supplemented with the initial conditions. We recall that to ensure stability, the explicit time integration leap-frog scheme must satisfy the usual Courant-Friedrichs-Levy (CFL) condition that imposes a restriction on $\Delta t$ of the form

$$
\Delta t \leq C_{\mathrm{CFL}}\left(c_{P}, \sigma_{0}\right) \frac{h}{p^{2}}
$$

where $h$ is the maximum mesh size and $p$ is the polynomial approximation degree (supposed to be uniform here, for the sake of simplicity). The constant $C_{\mathrm{CFL}}$ depends on the compressional wave velocity $c_{P}=\sqrt{(\lambda+2 \mu) / \rho}$ and on the stability parameter $\sigma_{0}$, cf. (24), and can be estimated as in [26], cf., also,[29].

\subsubsection{Example 1: Smooth problem with a known analytical solution}

We first consider the wave propagation problem in $\Omega=(0,1)^{2}$, where $\Gamma_{N}=(0,1) \times\{1\}, \Gamma_{D}=\partial \Omega \backslash \Gamma_{N}, \lambda=\mu=$ $\rho=1$ and boundary conditions, initial conditions and the forcing term $\mathbf{f}$ are selected so that the analytical solution of (19) is given by 

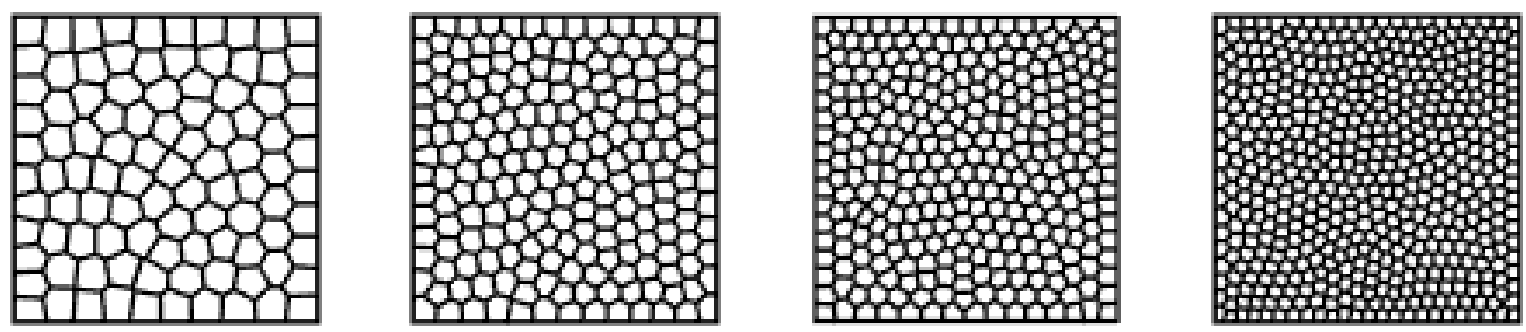

Fig. 13 Example 1 (Section 4.3.1). Mesh configurations considered with increasing number of polygonal elements: $N_{e l}=$ $100,200,300,500$.
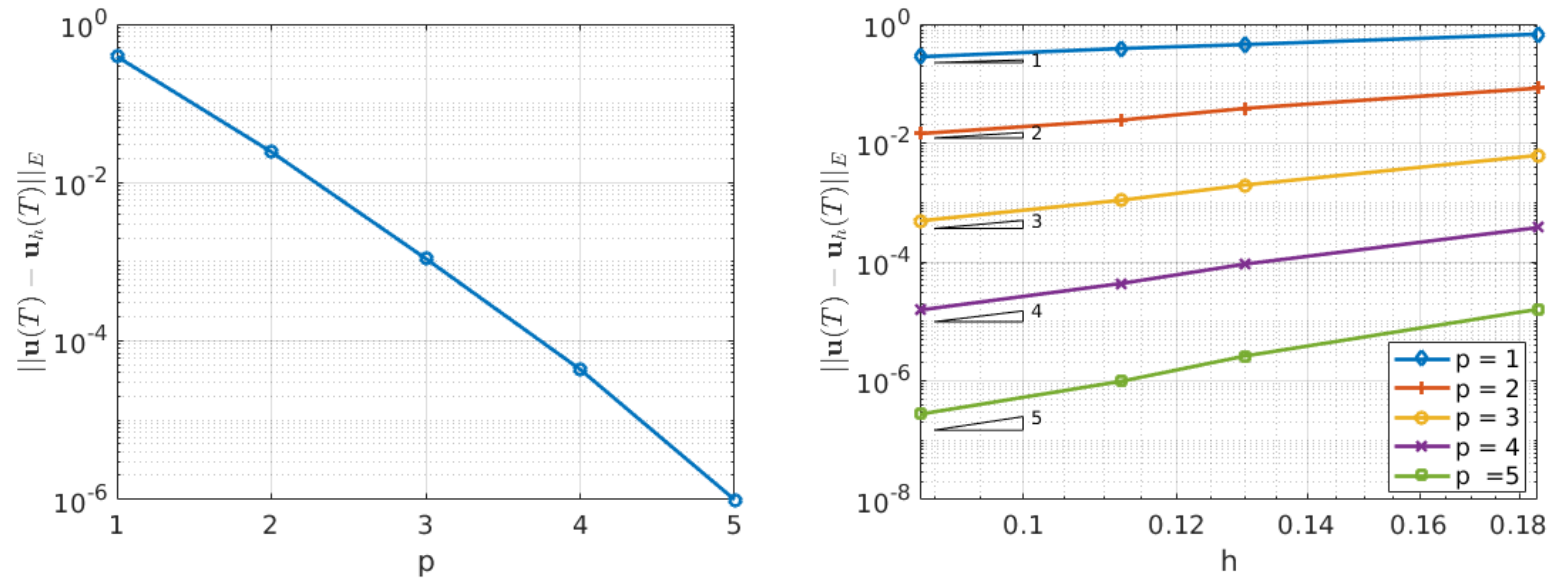

Fig. 14 Example 1 (Section 4.3.1). Computed error $\left\|\mathbf{u}(T)-\mathbf{u}_{h}(T)\right\|_{\mathrm{E}}$ versus the polynomial degree $p$, fixing $N_{e l}=300$ (left) and versus the mesh size $h=1 / N_{e l}, N_{e l}=100,200,300,500$ (right) fixing $p=2,3,4,5$. Results are obtained choosing as observation time $T=0.6$ with $\Delta t=10^{-5}$.

$$
\mathbf{u}(\mathbf{x}, t)=\cos (\sqrt{2} \pi t)\left[\begin{array}{r}
-\sin (\pi x) \sin (\pi y)^{2} \\
\cos (\pi x) \sin (\pi y)^{2}
\end{array}\right] .
$$

For the proceeding computations we set the final time $T=0.6$ and time step $\Delta t=10^{-5}$. Firstly, we consider the convergence of the PolyDG method with $p$-refinement. To this end, in Figure 14 (left) we plot $\left\|\mathbf{u}(T)-\mathbf{u}_{h}(T)\right\|_{\mathrm{E}}$ versus the polynomial degree $p_{E}=p$, for all $E \in \mathscr{T}_{h}$, on a fixed polygonal mesh $\mathscr{T}_{h}$ consisting of 300 elements; cf. Figure 13. Here, on a semi-logarithmic scale, we observe that the convergence line is approximately straight, thereby indicating exponential convergence of the PolyDG method as $p$ is uniformly enriched. Secondly, we consider the $h$ convergence of the PolyDG approximation computed on the sequence of meshes depicted in Figure 13. In Figure 14 (right), we observe that $\left\|\mathbf{u}(T)-\mathbf{u}_{h}(T)\right\|_{\mathrm{E}}$ behaves like $\mathscr{O}\left(h^{p}\right)$ as $h$ tends to zero, for each fixed $p$; this is in agreement with the a priori error bound stated in Theorem 1.

\subsubsection{Example 2: Elastic wave propagation in a heterogeneous medium}

For an application of the presented PolyDG method, we study the elastic wave propagation in the computational domain $\Omega=(0,38.4) \mathrm{km} \times(0,10) \mathrm{km}$ representing an idealized bidimensional Earth's cross section, see Figure 15 . The bottom and the lateral boundaries are set far enough from the point source (white dot in Figure 15) in order to prevent any reflections from the boundaries of the waves of interest. At the top of the model a free-surface boundary condition is imposed, i.e., $\sigma \mathbf{n}=\mathbf{0}$, whereas homogeneous Dirichlet conditions are set in the remaining part of the boundary. We simulate a point source load of the form 


$$
\mathbf{f}(\mathbf{x}, t)=\left(0, A e^{-10^{-4}\left\|\mathbf{x}-\mathbf{x}_{s}\right\|^{2}}\left(1-2 \pi^{2} f_{0}^{2}\left(t-t_{0}\right)^{2}\right) e^{-\pi^{2} f_{0}^{2}\left(t-t_{0}\right)^{2}}\right),
$$

with $A=10^{3} \mathrm{~N}, f_{0}=2 \mathrm{~Hz}$ and $t_{0}=2 \mathrm{~s}$ applied at the point $\mathbf{x}_{s}=(19.4,7.8) \mathrm{km}$. We assign constant material prop-

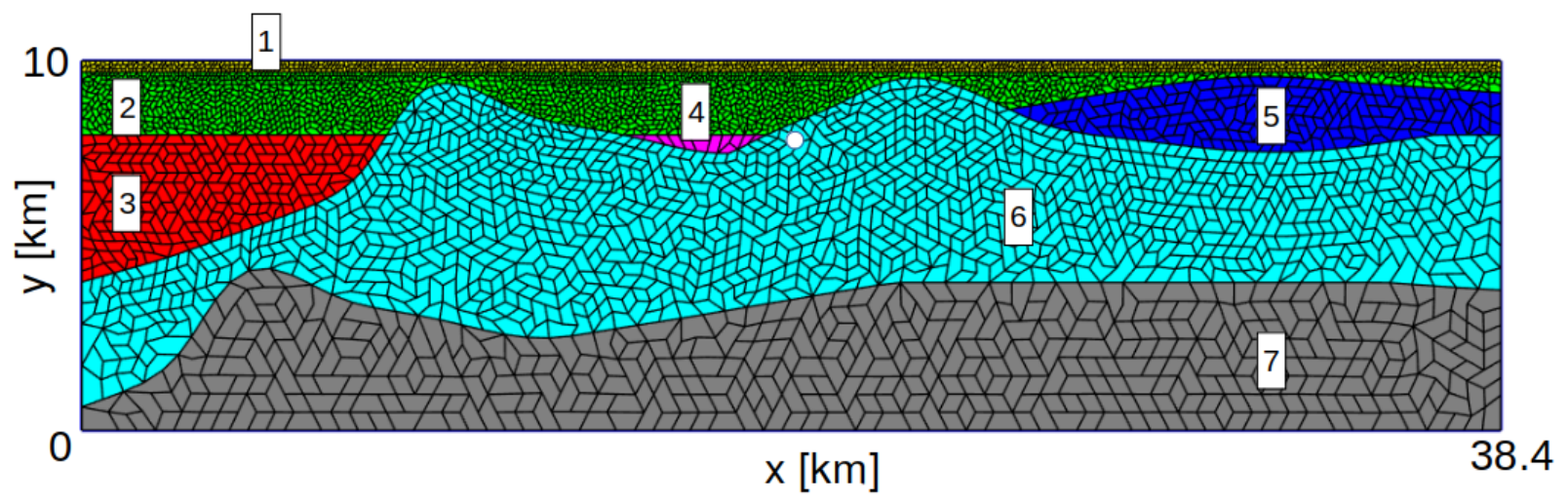

Fig. 15 Example 2 (Section 4.3.2). Unstructured polygonal grid. The mesh spacing varies from $h_{E} \approx 160 \mathrm{~m}$ for material 1 to $h_{E} \approx 1500 \mathrm{~m}$ for material 7; cf. Table 3. The source location $\mathbf{x}_{s}=(19.4,7.8) \mathrm{km}$ is indicated by a white circle.

erties within each region as described in Table 3. The computational domain is discretized using an unstructured grid

Table 3 Example 2 (Section 4.3.2). Material properties used for the computational domain in Figure 15.

\begin{tabular}{|c|c|c|c|}
\hline Material $\rho$ & {$\left[\mathrm{kg} / \mathrm{m}^{3}\right]$} & $c_{p}[\mathrm{~m} / \mathrm{s}]$ & $c_{s}[\mathrm{~m} / \mathrm{s}]$ \\
\hline 1 & 1800 & 1321 & 294 \\
\hline 2 & 1800 & 2024 & 450 \\
\hline 3 & 2050 & 1920 & 600 \\
\hline 4 & 2050 & 1920 & 650 \\
\hline 5 & 2050 & 2000 & 650 \\
\hline 6 & 2400 & 3030 & 1515 \\
\hline 7 & 2450 & 3200 & 1600 \\
\hline
\end{tabular}

consisting of 4870 (agglomerated) polygonal elements, with a mesh size varying from $h_{E} \approx 160 \mathrm{~m}$ for material 1 to $h_{E} \approx 1500 \mathrm{~m}$ for material 7 ; cf. Table 3 . The grid spacing is chosen small enough not only to describe with sufficient precision the physical profile of the submerged topography, but also to guarantee that over the whole domain there is at least 5 points per wavelength with polynomial degree equal to 4 to keep numerical dispersion and dissipation errors sufficiently small, i.e., of order of machine precision, see [26]. In Figure 16 we report a set of snapshots of the diplacement magnitude $|\mathbf{u}|=\sqrt{\mathbf{u}_{1}^{2}+\mathbf{u}_{2}^{2}}$ computed with the proposed method (with $\sigma_{0}=10$ and polynomial degree equal to 4) coupled with the leap-frog scheme, fixing the final observation time $T=5 \mathrm{~s}$ and time step $\Delta t=10^{-4} \mathrm{~s}$. The discontinuities between the mechanical properties of the materials produce oscillations and perturbations on the wave front. In particular, due to the stratigraphy of the model, the energy is focussed towards the left of the domain, reaches the surface of the model and (most of it) remains trapped within the first layer. All these complex and relevant phenomena are well captured by the proposed PolyDG method, see Figure 16.

\section{PolyDG methods for flow in fractured porous media}

The aim of this section is to present an overview of the results presented in [16], where a unified formulation and analysis of PolyDG approximations of flows in fractured porous media is provided for all primal-primal, primal-mixed, mixed-primal and mixed-mixed formulations. More precisely, a primal-primal setting consists of having the pressure as only unknown for both the bulk and fracture problems. When dealing with the approximation of Darcy's flow, one may 

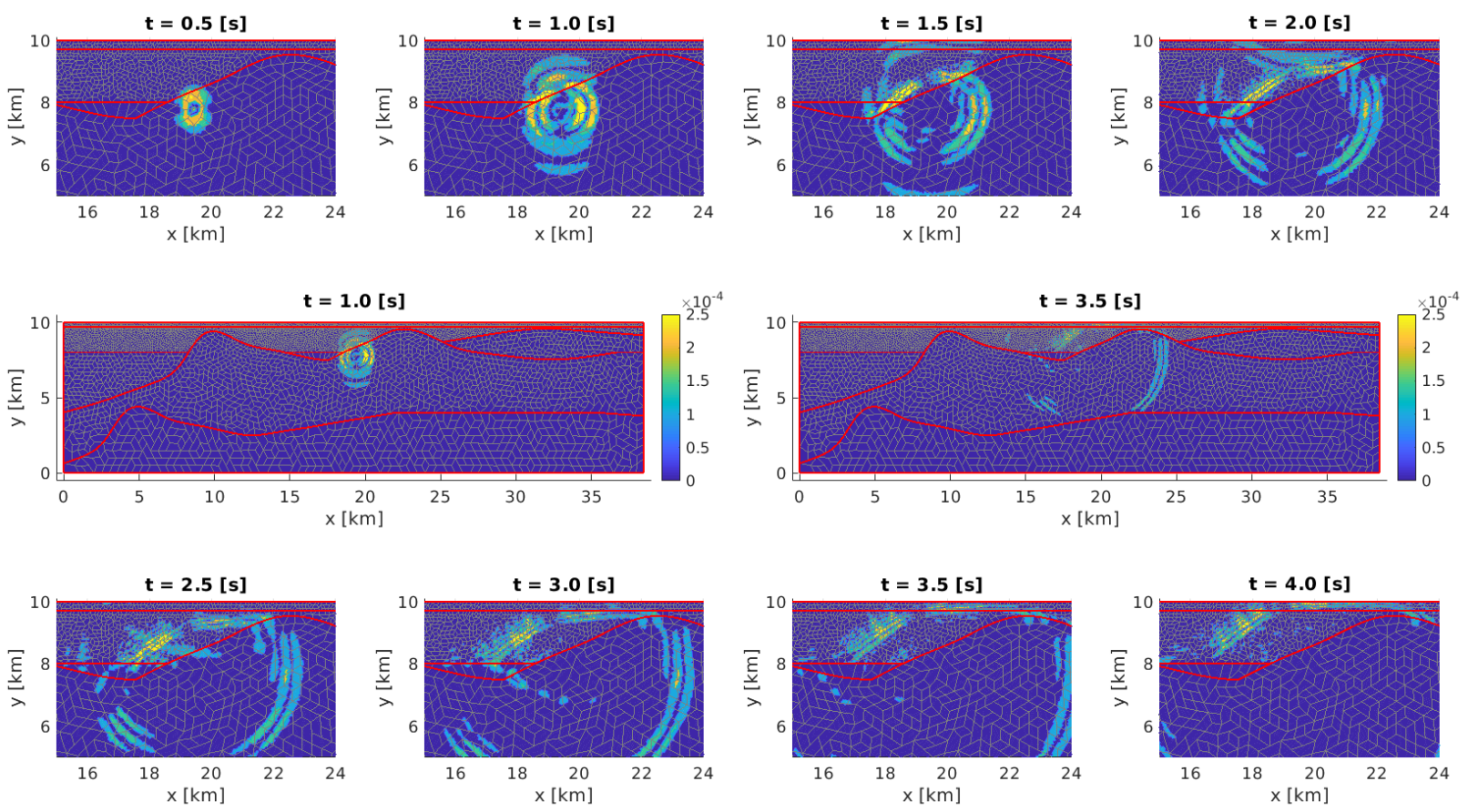

Fig. 16 Example 2 (Section 4.3.2). Snapshots of the computed displacement magnitude $|\mathbf{u}|=\sqrt{\mathbf{u}_{1}^{2}+\mathbf{u}_{2}^{2}}$ at different time $t=$ $0.5,1,1.5,2,2.5,3,3.5,4 \mathrm{~s}$. Due to the material heterogeneities, high oscillations and perturbations of the wave front can be observed. Waves moving leftwards with respect to the point source location are clearly visible. The displacement magnitude is measured in meters.

also resort to a mixed-mixed approach, where the flow is described through an additional unknown representing the (averaged) velocity of the fluid in both the bulk and the fracture. This variable, often referred to as Darcy's velocity, is of primary interest in many engineering applications [119,57], so that the mixed setting is often preferred to the primal one, which may only return the velocity after post-processing the computed pressure, thus entailing a potential loss of accuracy. On the other hand, the primal-primal approach is easier to solve, featuring a smaller number of degrees of freedom. For this reason, our aim is to design a unified setting where, according to the desired approximation properties of the model, one may resort to either a primal or mixed approximation for the problem in the bulk, as well as to a primal or mixed approximation for the problem in the fracture. In particular, for the primal discretizations we employ the Symmetric Interior Penalty discontinuous Galerkin method [148, 32], whereas for the mixed discretizations we employ the local DG (LDG) method of [79], both in their generalization to polytopic grids [67, 64, 13, 63, 66]. Our main reference for the design of such a setting is the work by Arnold et al. [33], where a unified analysis of all DG methods present in the literature is undertaken. This framework is based on the flux-formulation, where the so-called numerical fluxes are introduced on elemental interfaces as approximations of the analytical solution. Different choices of the numerical fluxes affect the stability and the accuracy of the underlying PolyDG method and provide conservation properties of desired quantities such as, for example, mass, momentum, and energy [66]. In the particular context of flow in fractured porous media, we also show that the coupling conditions between bulk and fracture problems may be imposed through a suitable definition of the numerical fluxes on the fracture faces. Such an abstract setting allows us to analyze theoretically, in a unified manner, all the possible combinations of primal-primal (PP), mixed-primal (MP), primal-mixed (PM) and mixed-mixed (MM) formulations for the bulk and fracture problems, respectively.

The rest of the section is organized as follows. In Section 5.1 we introduce the model problem; the discretization based on employing PolyDG methods is presented, in the unified setting of [33], in Section 5.2. In Section 5.3, we revise the main theoretical results, namely well-posedness and stability, and present $a$ priori error bounds. Illustrative numerical tests are presented in Section 5.4 to confirm the theoretical bounds. Moreover, we assess the capability of the method in handling more complicated geometries, presenting some test cases featuring networks of partially immersed fractures. 


\subsection{Model problem}

To describe the flow, which we assume to be single-phase flow, we adopt the mathematical model of [118]. This model was first introduced in $[3,2]$ for fractures with large permeability and is here generalised to handle also the low permeable case. An extension to two-phase flows can be found in $[99,107]$. To keep the presentation as simple as possible, we assume that the porous medium is cut by a single, non immersed fracture. We refer to [4] for the extension of the model to totally immersed fractures. Finally, in order to handle networks of intersecting fractures, some physical conditions need to be added to describe the behavior of the flow at the intersection points/lines. A possible choice is to impose pressure continuity and balance of fluxes as in $[96,56]$. Other, more general conditions, where the angle between fractures is taken into account and jumps of pressure across the intersection are allowed, may be found, for example, in $[95,133]$.

In the following we assume that the porous matrix is represented by the open, bounded, and polygonal/polyhedral domain $\Omega \subset \mathbb{R}^{d}, d=2,3$ and the fracture is described by the $(d-1)$-dimensional $\mathscr{C}^{\infty}$ manifold (with no curvature) $\Gamma \subset \mathbb{R}^{d-1}, d=2,3$. Since we are assuming that $\Gamma$ is not immersed, it separates $\Omega$ into the two connected disjoint subdomains $\Omega_{1}$ and $\Omega_{2}$. We decompose the boundary of $\Omega$ into two disjoint subsets $\partial \Omega_{D}$ and $\partial \Omega_{N}$, i.e., $\partial \Omega=$ $\partial \Omega_{D} \cup \partial \Omega_{N}$, with $\partial \Omega_{D} \cap \partial \Omega_{N}=\emptyset$, and we define $\partial \Omega_{D, i}=\partial \Omega_{D} \cap \partial \Omega_{i}$ and $\partial \Omega_{N, i}=\partial \Omega_{N} \cap \partial \Omega_{i}$, for $i=1$, 2. For the fracture domain we set $\partial \Gamma=\Gamma \cap \partial \Omega$ with $\partial \Gamma=\partial \Gamma_{D} \cup \partial \Gamma_{N}$. Finally, we denote by $\mathbf{n}_{\Gamma}$ the normal unit vector on $\Gamma$ with a fixed orientation from $\Omega_{1}$ to $\Omega_{2}$. Our model considers Darcy's flow in its mixed form for the problem both in the bulk and the fracture. More precisely, in addiction to the Darcy's pressure, we take into account an auxiliary vectorvalued variable, called Darcy's velocity. This quantity is of primary interest in many engineering applications, such as oil recovery and groundwater pollution modeling. Indeed, in these cases, in order to be effective, the simulation of the phenomenon requires very accurate approximation of the velocities of the involved fluids. The coupled bulk-fracture model problem in mixed form is given by:

$$
\begin{aligned}
& \mathbf{u}_{i}=v_{i} \nabla p_{i} \quad \text { in } \Omega_{i}, \\
& -\nabla \cdot \mathbf{u}_{i}=f_{i} \quad \text { in } \Omega_{i}, \\
& p_{i}=0 \quad \text { on } \partial \Omega_{D, i}, \\
& \mathbf{u}_{i} \cdot \mathbf{n}_{i}=0 \\
& \mathbf{u}_{\Gamma}=v_{\Gamma}^{\tau} \ell_{\Gamma} \nabla_{\tau} p_{\Gamma} \\
& -\nabla_{\tau} \cdot \mathbf{u}_{\Gamma}=\ell_{\Gamma} f_{\Gamma}-\llbracket \mathbf{u} \rrbracket \\
& p_{\Gamma}=0 \\
& \mathbf{u}_{\Gamma} \cdot \tau=0 \\
& -\{\mathbf{u}\} \cdot \mathbf{n}_{\Gamma}=\beta_{\Gamma} \llbracket p \rrbracket \cdot \mathbf{n}_{\Gamma} \\
& \text { on } \partial \Omega_{N, i} \\
& \text { in } \Gamma \text {, } \\
& \text { in } \Gamma \text {, } \\
& \text { on } \partial \Gamma_{D} \text {, } \\
& \text { on } \partial \Gamma_{N} \text {, } \\
& \text { on } \Gamma \text {, } \\
& -\llbracket \mathbf{u} \rrbracket=\alpha_{\Gamma}\left(\{p\}-p_{\Gamma}\right) \\
& \text { on } \Gamma \text {. }
\end{aligned}
$$

In the bulk, in each domain $\Omega_{i}, i=1,2$, the motion of an incompressible fluid with pressure $p_{i}$ and velocity $\mathbf{u}_{i}$ is described by (29a)-(29b), supplemented by the boundary conditions (29c)-(29d). Moreover, $f_{i} \in L^{2}\left(\Omega_{i}\right)$ represents a source term, and $v_{i}=v_{i}(x) \in \mathbb{R}^{d \times d}$ is the bulk permeability tensor, which we assume to be symmetric, positive definite, uniformly bounded from below and above and with entries that are bounded, piecewise continuous real-valued functions. Denoting by $p_{\Gamma}$ and $\mathbf{u}_{\Gamma}$ the fracture pressure and velocity, respectively, on the manifold $\Gamma$ representing the fracture, we formulate a reduced version of Darcy's law in the tangential direction, cf. equations (29e)-(29f), and assume that the fracture permeability tensor $v_{\Gamma}$, has a block-diagonal structure when written in its normal and tangential components and that $v_{\Gamma}^{\tau} \in \mathbb{R}^{(d-1) \times(d-1)}$ is positive definite and uniformly bounded. Moreover, $v_{\Gamma}$ satisfies the same regularity assumptions as those satisfied by the bulk permeability $v$. In (29e)-(29f)-(29g)-(29h), $f_{\Gamma} \in L^{2}(\Gamma)$, $\tau$ is the vector in the tangent plane of $\Gamma$ normal to $\partial \Gamma$ and $\nabla_{\tau}$ and $\nabla_{\tau}$. denote the tangential gradient and divergence operators, respectively. Finally, we close the model providing the interface conditions (29i) $-(29 \mathrm{j})$ where $\beta_{\Gamma}=\frac{1}{2 \eta_{\Gamma}}$, $\alpha_{\Gamma}=\frac{2}{\eta_{\Gamma}(2 \xi-1)}$ and $\eta_{\Gamma}=\frac{\ell_{\Gamma}}{v_{\Gamma}^{n}}, \ell_{\Gamma}>0$ being the fracture thickness. Finally, in the definition of $\alpha_{\Gamma}$, the closure parameter $\xi>1 / 2$ is related to the pressure profile across the fracture aperture. We refer to [118] for a rigorous derivation of the model.

To introduce the weak formulation, we first introduce the bulk pressure and velocity spaces: 
High-order DG methods on polyhedral grids for geophysical applications

$$
M^{b}=L^{2}(\Omega), \quad \mathbf{V}^{b}=\left\{\mathbf{v} \in H_{d i v}(\Omega):\left.\llbracket \mathbf{v} \rrbracket\right|_{\Gamma} \in L^{2}(\Gamma),\left.\{\mathbf{v}\}\right|_{\Gamma} \in\left[L^{2}(\Gamma)\right]^{d},\left.\mathbf{v} \cdot \mathbf{n}\right|_{\partial \Omega_{N}}=0\right\} .
$$

Similarly, for the fracture pressure and velocity we define the spaces

$$
M^{\Gamma}=L^{2}(\Gamma), \quad \mathbf{V}^{\Gamma}=\left\{\mathbf{v}_{\Gamma} \in H_{d i v, \tau}(\Gamma):\left.\mathbf{v}_{\Gamma} \cdot \tau\right|_{\partial \Gamma}=0\right\} .
$$

We equip the spaces $\mathbf{V}^{b}$ and $\mathbf{V}^{\Gamma}$ with the norms

$$
\begin{aligned}
\|\mathbf{v}\|_{\mathbf{v}^{b}}^{2} & =\|\mathbf{v}\|_{L^{2}(\Omega)}^{2}+\|\nabla \cdot \mathbf{v}\|_{L^{2}(\Omega)}^{2}+\|\llbracket \mathbb{v} \rrbracket\|_{L^{2}(\Gamma)}^{2}+\|\{\mathbf{v}\}\|_{L^{2}(\Gamma)}^{2}, \\
\left\|\mathbf{v}_{\Gamma}\right\|_{\mathbf{V}^{\Gamma}}^{2} & =\left\|\mathbf{v}_{\Gamma}\right\|_{L^{2}(\Gamma)}^{2}+\left\|\nabla_{\tau} \cdot \mathbf{v}_{\Gamma}\right\|_{L^{2}(\Gamma)}^{2}
\end{aligned}
$$

respectively. Finally, we define the global spaces for the pressure and the velocity as $M=M^{b} \times M^{\Gamma}$ and $\mathbf{W}=\mathbf{V}^{b} \times \mathbf{V}^{\Gamma}$, respectively, equipped with the canonical norms for product spaces. We can now formulate problem (29) in weak form as follows: find $\left(\mathbf{u}, \mathbf{u}_{\Gamma}\right) \in \mathbf{W}$ and $\left(p, p_{\Gamma}\right) \in M$ such that

$$
\begin{aligned}
A\left(\left(\mathbf{u}, \mathbf{u}_{\Gamma}\right),\left(\mathbf{v}, \mathbf{v}_{\Gamma}\right)\right)+B\left(\left(\mathbf{v}, \mathbf{v}_{\Gamma}\right),\left(p, p_{\Gamma}\right)\right) & =\mathbf{0}, \\
-B\left(\left(\mathbf{u}, \mathbf{u}_{\Gamma}\right),\left(q, q_{\Gamma}\right)\right) & =F^{p}\left(q, q_{\Gamma}\right)
\end{aligned}
$$

for all $\left(\mathbf{v}, \mathbf{v}_{\Gamma}\right) \in \mathbf{W}$ and $\left(q, q_{\Gamma}\right) \in M$, where the bilinear forms $A(\cdot, \cdot): \mathbf{W} \times \mathbf{W} \rightarrow \mathbb{R}$ and $B(\cdot, \cdot): \mathbf{W} \times M \rightarrow \mathbb{R}$ are defined as

$$
\begin{aligned}
A\left(\left(\mathbf{u}, \mathbf{u}_{\Gamma}\right),\left(\mathbf{v}, \mathbf{v}_{\Gamma}\right)\right) & =a(\mathbf{u}, \mathbf{v})+a_{\Gamma}\left(\mathbf{u}_{\Gamma}, \mathbf{v}_{\Gamma}\right), \\
B\left(\left(\mathbf{v}, \mathbf{v}_{\Gamma}\right),\left(q, q_{\Gamma}\right)\right) & =b(\mathbf{v}, q)+b_{\Gamma}\left(\mathbf{v}_{\Gamma}, q_{\Gamma}\right)+d\left(\mathbf{v}, q_{\Gamma}\right),
\end{aligned}
$$

respectively, with

$$
\begin{aligned}
a(\mathbf{u}, \mathbf{v}) & =\int_{\Omega} v^{-1} \mathbf{u} \cdot \mathbf{v}+\int_{\Gamma} \frac{1}{\alpha_{\Gamma}} \llbracket \mathbf{u} \rrbracket \llbracket \mathbf{v} \rrbracket+\int_{\Gamma} \frac{1}{\beta_{\Gamma}}\{\mathbf{u}\} \cdot\{\mathbf{v}\}, \\
a_{\Gamma}\left(\mathbf{u}_{\Gamma}, \mathbf{v}_{\Gamma}\right) & =\int_{\Gamma}\left(v_{\Gamma}^{\tau} \ell_{\Gamma}\right)^{-1} \mathbf{u}_{\Gamma} \cdot \mathbf{v}_{\Gamma},
\end{aligned}
$$

and

$$
b(\mathbf{v}, q)=\int_{\Omega} \nabla \cdot \mathbf{v} q, \quad b_{\Gamma}\left(\mathbf{v}_{\Gamma}, q_{\Gamma}\right)=\int_{\Gamma} \nabla_{\tau} \cdot \mathbf{v}_{\Gamma} q_{\Gamma}, \quad d\left(\mathbf{v}, q_{\Gamma}\right)=-\int_{\Gamma} \llbracket \mathbf{v} \rrbracket q_{\Gamma} .
$$

Finally the linear operator $F^{p}(\cdot): M \rightarrow \mathbb{R}$ is defined as $F^{p}\left(q, q_{\Gamma}\right)=\int_{\Omega} f q+\int_{\Gamma} \ell_{\Gamma} f_{\Gamma} q_{\Gamma}$.

We next recall the following well-posedness result: we refer to [16] for the proof. Note that the existence and uniqueness of the problem can be proven only under the condition that the parameter $\xi>1 / 2$.

Theorem 2. Suppose that $\xi>1 / 2$, then problem (30) admits a unique solution.

\subsection{PolyDG discretization of flow in fractured porous media: a unified approach}

In this section we present, in a unified setting, a family of discrete formulations for the coupled bulk-fracture problem (30). In particular, the problem in the bulk and the one in the fracture can be either discretized in their mixed or primal form. We then derive four formulations that embrace all the possible combinations of primal-primal, mixed-primal, primal-mixed and mixed-mixed discretizations. The primal discretizations will be based on the Symmetric Interior Penalty DG method (SIPDG) [32, 148], while the mixed approach will exploit the Local Discontinuous Galerkin method (LDG) [79, 69, 128], including their extension to polytopic grids [67, 64, 13, 63, 66]. The derivation follows the approach of [33] based on the introduction of the numerical fluxes, which approximate the trace of the solutions on the boundary of each mesh element. In particular, the imposition of the coupling conditions (29i)-(29j) will be achieved through a proper definition of the numerical fluxes on the faces belonging to the fracture.

We consider a sequence of meshes $\mathscr{T}_{h}$ that is aligned with the fracture $\Gamma$ and we denote, as in Section 2, by $\mathscr{F}_{h}$ the set of all the faces of the mesh $\mathscr{T}_{h}$, that we can decompose as $\mathscr{F}_{h}=\mathscr{F}_{h} \cup \mathscr{F}_{h}^{B} \cup \Gamma_{h}$, where now $\mathscr{F}_{h} I$ is the set of 
Fig. 17 Example of two neighboring elements of a polygonal bulk mesh aligned with the fracture and of the induced subdivision.

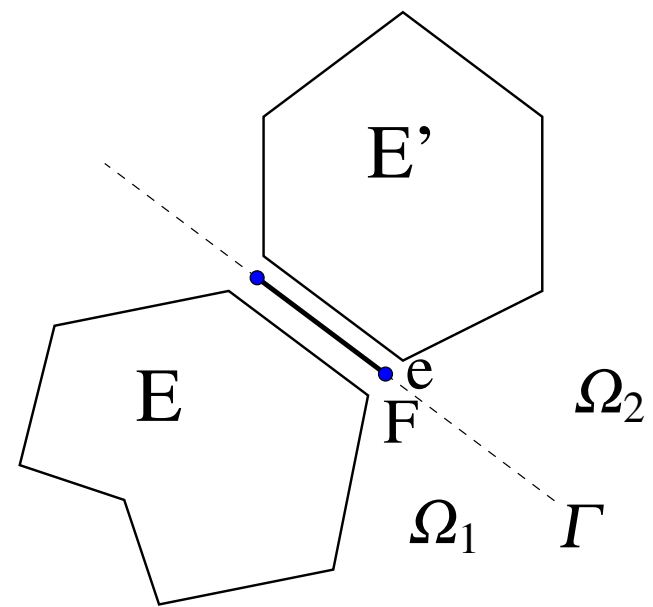

interior faces not belonging to the fracture, $\mathscr{F}_{h}^{B}$ is the set of faces lying on the boundary of the domain $\partial \Omega$ (which can be further decomposed into $\mathscr{F}_{h}^{B}=\mathscr{F}_{h}^{D} \cup \mathscr{F}_{h}^{N}$ ) and $\Gamma_{h}$ is the set of fracture faces. In particular, the induced subdivision of the fracture $\Gamma_{h}$ consists of the faces of the elements of $\mathscr{T}_{h}$ that share part of their boundary with the fracture, so that, according to the definition of $\mathscr{F}_{h}$ given in Section 2.1, $\Gamma_{h}$ is made up of line segments when $d=2$ and of triangles when $d=3$. In the latter case, the triangles are not necessarily shape-regular and they may present hanging nodes, due to the fact that the sub-triangulations of each elemental interface is chosen independently from the others. For this reason, we here extend the concept of interface introduced in Section 2.1 also to the $(d-2)$-dimensional facets of elements in $\Gamma_{h}$, defined again as intersection of boundaries of two neighbouring elements. When $d=2$, the interfaces reduce to points (see Figure 17), while when $d=3$ they consists of line segments. Moreover, since we aim at employing PolyDG methods also for the discretization of the problem in the fracture, we denote by $\mathscr{E}_{\Gamma, h}$ the set of all the interfaces (that we will also call edges) of the elements in $\Gamma_{h}$, and we write, accordingly to the previous notation, $\mathscr{E}_{\Gamma, h}=\mathscr{E}_{\Gamma, h} \cup \mathscr{E}_{\Gamma, h}^{B}$, with $\mathscr{E}_{\Gamma, h}^{B}=\mathscr{E}_{\Gamma, h} \cup \mathscr{E}_{\Gamma, h} N$. For the forthcoming stability and error analysis, we assume that both the bulk and fracture sequence of meshes are polytopic-regular, according to Assumption 2 and that the covering satisfies Assumption 4. Moreover, we suppose that the permeability tensors $v$ and $v_{\Gamma}$ are piecewise constant on mesh elements, i.e., $\left.v\right|_{E} \in\left[\mathbb{P}_{0}(E)\right]^{d \times d}$ for all $E \in \mathscr{T}_{h}$, and $\left.v_{\Gamma}\right|_{F} \in\left[\mathbb{P}_{0}(F)\right]^{(d-1) \times(d-1)}$ for all $F \in \Gamma_{h}$.

First, to each element $E \in \mathscr{T}_{h}$ and $F \in \Gamma_{h}$ we associate the integers $p_{E} \geq 1$ and $p_{F} \geq 1$, and introduce the finitedimensional spaces:

$$
\begin{aligned}
Q_{h}^{b} & =\left\{q \in L^{2}(\Omega):\left.q\right|_{E} \in \mathbb{P}_{p_{E}}(E) \forall E \in \mathscr{T}_{h}\right\}, \\
\mathbf{W}_{h}^{b} & =\left\{\mathbf{v} \in\left[L^{2}(\Omega)\right]^{d}:\left.\mathbf{v}\right|_{E} \in\left[\mathbb{P}_{p_{E}}(E)\right]^{d} \forall E \in \mathscr{T}_{h}\right\}, \\
Q_{h}^{\Gamma} & =\left\{q_{\Gamma} \in L^{2}(\Gamma):\left.q_{\Gamma}\right|_{F} \in \mathbb{P}_{p_{F}}(F) \forall F \in \Gamma_{h}\right\}, \\
\mathbf{W}_{h}^{\Gamma} & =\left\{\mathbf{v}_{\Gamma} \in\left[L^{2}(\Gamma)\right]^{d-1}:\left.\mathbf{v}_{\Gamma}\right|_{F} \in\left[\mathbb{P}_{p_{F}}(F)\right]^{d-1} \forall F \in \Gamma_{h}\right\} .
\end{aligned}
$$

We remark that the polynomial degrees in the bulk and fracture discrete spaces are chosen independently of each other.

We next focus on equations (29a)-(29b) in the bulk and equations (29e) -(29f) in the fracture. We proceed as in [33], and multiply equations (29a)-(29b) by (sufficiently smooth) vector-valued and scalar-valued test functions, respectively, integrate by parts over an element $E \in \mathscr{T}_{h}$, and sum over all elements. Analogously, we multiply equations (29e)-(29f) by (sufficiently smooth) test functions, integrate by parts over an element $F \in \Gamma_{h}$ and sum over all the elements in $\Gamma_{h}$. We then discretize, use identity (4), and integrate by parts again the first equation in the bulk and the first equation in the fracture, to get the following general discrete formulation: find $p_{h} \in Q_{h}^{b}, \mathbf{u}_{h} \in \mathbf{W}_{h}^{b}, p_{\Gamma, h} \in Q_{h}^{\Gamma}$, and $\mathbf{u}_{\Gamma, h} \in \mathbf{W}_{h}^{\Gamma}$ such that 


\begin{tabular}{lll}
\hline Method & \multicolumn{1}{c}{ Primal bilinear form } & Reference equations \\
\hline Primal-Primal (PP) & $\mathscr{A}_{b}^{P}(p, q)+\mathscr{A}_{\Gamma}^{P}\left(p_{\Gamma}, q_{\Gamma}\right)+\mathscr{C}\left((p, q),\left(p_{\Gamma}, q_{\Gamma}\right)\right)$ & $(33),(34),(35)$ \\
Mixed-Primal (MP) & $\mathscr{A}_{b}^{M}(p, q)+\mathscr{A}_{\Gamma}^{P}\left(p_{\Gamma}, q_{\Gamma}\right)+\mathscr{C}\left((p, q),\left(p_{\Gamma}, q_{\Gamma}\right)\right)$ & $(41),(34),(35)$ \\
Primal-Mixed (PM) & $\mathscr{A}_{b}^{P}(p, q)+\mathscr{A}_{\Gamma}^{M}\left(p_{\Gamma}, q_{\Gamma}\right)+\mathscr{C}\left((p, q),\left(p_{\Gamma}, q_{\Gamma}\right)\right)$ & $(33),(46),(35)$ \\
Mixed-Mixed (MM) & $\mathscr{A}_{b}^{M}(p, q)+\mathscr{A}_{\Gamma}^{M}\left(p_{\Gamma}, q_{\Gamma}\right)+\mathscr{C}\left((p, q),\left(p_{\Gamma}, q_{\Gamma}\right)\right)$ & $(41),(46),(35)$ \\
\hline
\end{tabular}

Table 4 Primal forms for the DG discretizations of the bulk-fracture problems.

$$
\begin{aligned}
& \int_{\mathscr{T}_{h}} v^{-1} \mathbf{u}_{h} \cdot \mathbf{v}=\int_{\mathscr{T}_{h}} \nabla p_{h} \cdot \mathbf{v}+\int_{\mathscr{F}_{h}^{I} \cup \Gamma_{h}}\left\{\hat{p}-p_{h}\right\} \llbracket \mathbf{v} \rrbracket+\int_{\mathscr{F}_{h}^{I} \cup \mathscr{F}_{h}^{B} \cup \Gamma_{h}} \llbracket \hat{p}-p_{h} \rrbracket \cdot\{\mathbf{v}\}, \\
& \int_{\mathscr{T}_{h}} \mathbf{u}_{h} \cdot \nabla q-\int_{\mathscr{F}_{h}^{I} \cup \mathscr{F} B}\{\hat{\mathbf{u}}\} \cdot \llbracket q \rrbracket-\int_{\mathscr{F}} \llbracket \hat{\mathbf{u}}_{h} \rrbracket\{q\}=\int_{\mathscr{T}_{h}} f q, \\
& \int_{\Gamma_{h}}\left(v_{\Gamma}^{\tau} \ell_{\Gamma}\right)^{-1} \mathbf{u}_{\Gamma, h} \cdot \mathbf{v}_{\Gamma}=\int_{\Gamma_{h}} \nabla p_{\Gamma, h} \cdot \mathbf{v}_{\Gamma}+\int_{\mathscr{E}_{\Gamma, h}^{I}}\left\{\hat{p}_{\Gamma}-\hat{p}_{\Gamma, h}\right\} \llbracket \mathbf{v}_{\Gamma} \rrbracket+\int_{\mathscr{E}_{\Gamma, h}} \llbracket \hat{p}_{\Gamma}-\hat{p}_{\Gamma, h} \rrbracket \cdot\left\{\mathbf{v}_{\Gamma}\right\}, \\
& \int_{\Gamma_{h}} \mathbf{u}_{\Gamma, h} \cdot \nabla q_{\Gamma}-\int_{\mathscr{E}_{\Gamma, h}}\left\{\hat{\mathbf{u}}_{\Gamma}\right\} \cdot \llbracket q_{\Gamma} \rrbracket-\int_{\mathscr{E}_{\Gamma, h}} \llbracket \hat{\mathbf{u}}_{\Gamma} \rrbracket\left\{q_{\Gamma}\right\}=\int_{\Gamma_{h}} \ell_{\Gamma} f_{\Gamma} q_{\Gamma}-\int_{\Gamma_{h}} \llbracket \hat{u} \rrbracket q_{\Gamma}
\end{aligned}
$$

for all $q \in Q_{h}^{b}, \mathbf{v} \in \mathbf{W}_{h}^{b}, q_{\Gamma} \in Q_{h}^{\Gamma}$ and $\mathbf{v}_{\Gamma} \in \mathbf{W}_{h}^{\Gamma}$. We point out that, in order to simplify the notation, we have dropped the subscript $\tau$ from the tangent gradient and divergence operators. Here, in the spirit of [33], the numerical fluxes

$$
\hat{p}=\left(\hat{p}_{E}\right)_{E \in \mathscr{T}_{h}}, \quad \hat{\mathbf{u}}=\left(\hat{\mathbf{u}}_{E}\right)_{E \in \mathscr{T}_{h}}, \quad \hat{p}_{\Gamma}=\left(\hat{p}_{\Gamma, F}\right)_{F \in \Gamma_{h}}, \quad \hat{\mathbf{u}}_{\Gamma}=\left(\hat{\mathbf{u}}_{\Gamma, F}\right)_{F \in \Gamma_{h}},
$$

are approximations to the analytical solutions $\mathbf{u}$ and $p$, respectively, on the boundary of $E$ and to $p_{\Gamma}$ and $\mathbf{u}_{\Gamma}$, respectively, on the boundary of the fracture face $F$. The numerical fluxes $\hat{p}, \hat{\mathbf{u}}, \hat{p}_{\Gamma}, \hat{\mathbf{u}}_{\Gamma}$ must be interpreted as linear functionals taking values in the spaces $\Pi_{E \in \mathscr{T}_{h}} L^{2}(\partial E),\left[\Pi_{E \in \mathscr{T}_{h}} L^{2}(\partial E)\right]^{d}, \Pi_{F \in \Gamma_{h}} L^{2}(\partial F),\left[\Pi_{F \in \Gamma_{h}} L^{2}(\partial F)\right]^{d}$, respectively. By suitably choosing the numerical fluxes, we can obtain all the possible combinations of primal-primal, mixed-primal, primal-mixed and mixed-mixed formulations for the bulk and fracture, respectively. In Table 4 we summarize the bilinear forms for all formulations, whose precise definition will be given in the forthcoming sections.

\subsubsection{Primal-Primal formulation}

To obtain the primal-primal formulation, based on the symmetric interior penalty Discontinuous Galerkin (SIPDG) method, we choose the numerical fluxes $\hat{p}=\hat{p}\left(p_{h}\right), \hat{\mathbf{u}}=\hat{\mathbf{u}}\left(p_{h}, p_{\Gamma, h}\right), \hat{p}_{\Gamma}=\hat{p}_{\Gamma}\left(p_{\Gamma, h}\right)$, and $\hat{\mathbf{u}}_{\Gamma}=\hat{\mathbf{u}}_{\Gamma}\left(p_{\Gamma, h}\right)$ as follows

$$
\begin{array}{rlrl}
\hat{p} & =\left\{\begin{array}{lll}
\left\{p_{h}\right\} & \text { on } \mathscr{F}_{h}^{I} \\
0 & \text { on } \mathscr{F}_{h}^{D} \\
p_{h} & \text { on } \mathscr{F}_{h}^{N} \\
p_{h} & \text { on } \Gamma_{h}
\end{array}\right. & \begin{cases}\left\{v \nabla p_{h}\right\}-\sigma_{F} \llbracket p_{h} \rrbracket & \text { on } \mathscr{F} h \\
v \nabla p_{h}-\sigma_{F} p_{h} \mathbf{n}_{F} & \text { on } \mathscr{F}_{h}^{D} \\
0 & \text { on } \mathscr{F}_{h}^{N} \\
-\left[\alpha_{\Gamma}\left(\left\{p_{h}\right\}-p_{\Gamma, h}\right) \frac{\mathbf{n}_{F}}{2}+\beta_{\Gamma} \llbracket p_{h} \rrbracket\right] & \text { on } \Gamma_{h}\end{cases} \\
\hat{p}_{\Gamma}=\left\{\begin{array}{lll}
\left\{p_{\Gamma, h}\right\} & \text { on } \mathscr{E}_{\Gamma, h}^{I} \\
0 & \text { on } \mathscr{E}_{\Gamma, h}^{D} \\
p_{\Gamma, h} & \text { on } \mathscr{E}_{\Gamma, h}^{N},
\end{array}\right. & \hat{\mathbf{u}}_{\Gamma}= \begin{cases}\left\{v_{\Gamma}^{\tau} \ell_{\Gamma} \nabla p_{\Gamma, h}\right\}-\sigma_{e} \llbracket p_{\Gamma, h} \rrbracket & \text { on } \mathscr{E}_{\Gamma, h}^{I} \\
v_{\Gamma}^{\tau} \ell_{\Gamma} \nabla p_{\Gamma, h}-\sigma_{e} p_{\Gamma, h} \mathbf{n}_{e} & \text { on } \mathscr{E}_{\Gamma, h}^{D} \\
0 & \text { on } \mathscr{E}_{\Gamma, h}^{N} .\end{cases}
\end{array}
$$

Here, we have introduced the discontinuity penalization parameters $\sigma$ and $\sigma_{\Gamma} \in L^{\infty}\left(\mathrm{e}^{I} \cup \mathrm{e}^{D}\right)$. In particular, they are nonnegative bounded functions and their precise definitions will be given in Definition 5 below. Moreover, we have used the notation $\sigma_{F}=\left.\sigma\right|_{F}$, for $F \in \mathscr{F}_{h}^{I} \cup \mathscr{F}_{h}^{D}$ and $\sigma_{e}=\left.\sigma_{\Gamma}\right|_{e}$ for $e \in \mathrm{e}^{I} \cup \mathrm{e}^{D}$. Note also that, with this choice, the numerical flux $\hat{p}$ is double valued on $\Gamma_{h}$ and single valued on $\mathscr{F}_{h} \cup \mathscr{F}_{h}^{B}$. By using the above definitions, and after eliminating the velocities $\mathbf{u}_{h}$ and $\mathbf{u}_{\Gamma, h}$ in an elementwise manner as in [33], based on the fact that $\nabla Q_{h} \subseteq \mathbf{W}_{h}, \nabla Q_{h}^{\Gamma} \subseteq \mathbf{W}_{h}^{\Gamma}$ and 
employing the lifting operators

$$
\begin{array}{lll}
\mathscr{L}_{b}^{S I P}:\left[L^{1}\left(\mathscr{F}_{h}^{I} \cup \mathscr{F}_{h}^{D}\right)\right]^{d} \rightarrow \mathbf{W}_{h}^{b}, & \int_{\Omega} \mathscr{L}_{b}^{S I P}(\xi) \cdot \mathbf{v}=-\int_{\mathscr{F}_{h}^{I} \cup \mathscr{F}_{h}^{D}}\{\mathbf{v}\} \cdot \xi & \forall \mathbf{v} \in \mathbf{W}_{h}^{b}, \\
\mathscr{L}_{\Gamma}^{S I P}:\left[L^{1}\left(\mathscr{E}_{\Gamma, h}^{I} \cup \mathrm{e}^{D}\right)\right]^{d-1} \rightarrow \mathbf{W}_{h}^{\Gamma}, & \int_{\Gamma} \mathscr{L}_{\Gamma}^{S I P}\left(\xi_{\Gamma}\right) \cdot \mathbf{v}_{\Gamma}=-\int_{\mathscr{E}_{\Gamma, h}{ }^{\perp} \mathrm{e}^{D}}\left\{\mathbf{v}_{\Gamma}\right\} \cdot \xi_{\Gamma} & \forall \mathbf{v}_{\Gamma} \in \mathbf{W}_{h}^{\Gamma},
\end{array}
$$

we obtain the following discrete formulation: find $\left(p_{h}, p_{h}^{\Gamma}\right) \in Q_{h}^{b} \times Q_{h}^{\Gamma}$ such that

$$
\mathscr{A}_{h}^{P P}\left(\left(p_{h}, p_{h}^{\Gamma}\right),\left(q, q_{\Gamma}\right)\right)=\mathscr{L}_{h}^{P P}\left(q, q_{\Gamma}\right) \quad \forall\left(q, q_{\Gamma}\right) \in Q_{h}^{b} \times Q_{h}^{\Gamma},
$$

where the superscript $P P$ stands for primal-primal and $\mathscr{L}_{h}^{P P}: Q_{h}^{b} \times Q_{h}^{\Gamma} \rightarrow \mathbb{R}$ is defined as $\mathscr{L}_{h}^{P P}\left(q, q_{\Gamma}\right)=\mathscr{L}_{b}^{P}(q)+$ $\mathscr{L}_{\Gamma}^{P}\left(q_{\Gamma}\right)$ and $\mathscr{A}_{h}^{P P}:\left(Q_{h}^{b} \times Q_{h}^{\Gamma}\right) \times\left(Q_{h}^{b} \times Q_{h}^{\Gamma}\right) \rightarrow \mathbb{R}$ is given by

$$
\mathscr{A}_{h}^{P P}\left(\left(p_{h}, p_{h}^{\Gamma}\right),\left(q, q_{\Gamma}\right)\right)=\mathscr{A}_{b}^{P}\left(p_{h}, q\right)+\mathscr{A}_{\Gamma}^{P}\left(p_{\Gamma, h}, q_{\Gamma}\right)+\mathscr{C}\left(\left(p_{h}, p_{\Gamma, h}\right),\left(q, q_{\Gamma}\right)\right),
$$

with

$$
\begin{aligned}
& \mathscr{A}_{b}^{P}\left(p_{h}, q\right)=\int_{\mathscr{T}_{h}} v \nabla p_{h} \cdot \nabla q+\int_{\mathscr{T}_{h}} v \mathscr{L}_{b}^{S I P}\left(\llbracket p_{h} \rrbracket\right) \cdot \nabla q \\
& +\int_{\mathscr{T}_{h}} v \mathscr{L}_{b}^{S I P}(\llbracket q \rrbracket) \cdot \nabla p_{h}+\int_{\mathscr{F}_{h}^{I} \cup \mathscr{F}_{h}^{D}} \sigma_{F} \llbracket p_{h} \rrbracket \cdot \llbracket q \rrbracket, \\
& \mathscr{A}_{\Gamma}^{P}\left(p_{\Gamma, h}, q_{\Gamma}\right)=\int_{\Gamma_{h}} v_{\Gamma}^{\tau} \ell_{\Gamma} \nabla p_{\Gamma, h} \cdot \nabla q_{\Gamma}+\int_{\Gamma_{h}} v_{\Gamma}^{\tau} \ell_{\Gamma} \mathscr{L}_{\Gamma}^{P}\left(\llbracket p_{\Gamma, h} \rrbracket\right) \cdot \nabla q_{\Gamma} \\
& +\int_{\Gamma_{h}} v_{\Gamma}^{\tau} \ell_{\Gamma} \mathscr{L}_{\Gamma}^{S I P}\left(\llbracket q_{\Gamma} \rrbracket\right) \cdot \nabla p_{\Gamma, h}+\int_{\mathscr{E}_{\Gamma, h}^{I} \cup \mathscr{E}_{\Gamma, h}^{D}} \sigma_{e} \llbracket p_{\Gamma, h} \rrbracket \cdot \llbracket q_{\Gamma} \rrbracket, \\
& \mathscr{C}\left(\left(p_{h}, p_{\Gamma, h}\right),\left(q, q_{\Gamma}\right)\right)=\int_{\Gamma_{h}} \beta_{\Gamma} \llbracket p_{h} \rrbracket \cdot \llbracket q \rrbracket+\int_{\Gamma_{h}} \alpha_{\Gamma}\left(\left\{p_{h}\right\}-p_{\Gamma, h}\right)\left(\{q\}-q_{\Gamma, h}\right),
\end{aligned}
$$

and

$$
\mathscr{L}_{b}^{P}(q)=\int_{\mathscr{T} h} f q, \quad \mathscr{L}_{\Gamma}^{P}\left(q_{\Gamma}\right)=\int_{\Gamma_{h}} \ell_{\Gamma} f_{\Gamma} q_{\Gamma}
$$

\subsubsection{Mixed-Primal formulation}

We next address the choice of the numerical fluxes that leads to a mixed-primal formulation. Here, the mixed formulation will be based on the use of the LDG method [79, 69, 127, 128]. To this end, we define the numerical fluxes $\hat{p}=\hat{p}\left(p_{h}\right)$ and $\hat{\mathbf{u}}=\hat{\mathbf{u}}\left(\mathbf{u}_{h}, p_{h}, p_{\Gamma, h}\right)$ for the bulk as

$$
\hat{p}=\left\{\begin{array}{ll}
\left\{p_{h}\right\}+\mathbf{b} \cdot \llbracket p_{h} \rrbracket & \text { on } \mathscr{F}_{h}^{I}, \\
0 & \text { on } \mathscr{F}_{h}^{D}, \\
p_{h} & \text { on } \mathscr{F}_{h}^{N}, \\
p_{h} & \text { on } \Gamma_{h},
\end{array} \quad \hat{\mathbf{u}}= \begin{cases}\left\{\mathbf{u}_{h}\right\}-\mathbf{b} \llbracket \mathbf{u}_{h} \rrbracket-\sigma_{F} \llbracket p_{h} \rrbracket & \text { on } \mathscr{F}_{h}^{I}, \\
\mathbf{u}_{h}-\sigma_{F} p_{h} \mathbf{n}_{F} & \text { on } \mathscr{F}_{h} D, \\
0 & \text { on } \mathscr{F}_{h}^{N}, \\
-\left[\alpha_{\Gamma}\left(\left\{p_{h}\right\}-p_{\Gamma, h}\right) \frac{\mathbf{n}_{F}}{2}+\beta_{\Gamma} \llbracket p_{h} \rrbracket\right] & \text { on } \Gamma_{h},\end{cases}\right.
$$

whereas for the numerical fluxes in the fracture we adopt the same definition as in (31). Here, $\mathbf{b} \in\left[L^{\infty}\left(\mathscr{F}{ }_{h}\right)\right]^{d}$ is a (possibly null) facewise constant vector-valued function such that $\|\mathbf{b}\|_{\infty, \mathscr{F}_{h}^{I}} \lesssim 1$. With this definition of the numerical fluxes, we obtain the following discrete mixed problem: find $\left(\left(p_{h}, \mathbf{u}_{h}\right), p_{h}^{\Gamma}\right) \in Q_{h}^{b} \times \mathbf{W}_{h}^{b} \times Q_{h}^{\Gamma}$ such that 
High-order DG methods on polyhedral grids for geophysical applications

$$
\begin{aligned}
\mathscr{M}_{b}\left(\mathbf{u}_{h}, \mathbf{v}\right)+\mathscr{B}_{b}\left(p_{h}, \mathbf{v}\right) & =\mathbf{0} & & \forall \mathbf{v} \in \mathbf{W}_{h}^{b}, \\
-\mathscr{B}_{b}\left(q, \mathbf{u}_{h}\right)+\mathscr{S}_{b}\left(p_{h}, q\right)+\mathscr{C}_{1}\left(p_{h}, q, p_{\Gamma, h}\right) & =\mathscr{L}_{b}^{P}(q) & & \forall q \in Q_{h}^{b}, \\
\mathscr{A}_{\Gamma}^{P}\left(p_{\Gamma, h}, q_{\Gamma}\right)+\mathscr{C}_{2}\left(p_{h}, p_{\Gamma, h}, q_{\Gamma}\right) & =\mathscr{L}_{\Gamma}^{P}\left(q_{\Gamma}\right) & & \forall q_{\Gamma} \in Q_{h}^{\Gamma},
\end{aligned}
$$

where

$$
\begin{aligned}
\mathscr{M}_{b}\left(\mathbf{u}_{h}, \mathbf{v}\right) & =\int_{\mathscr{T}_{h}} v^{-1} \mathbf{u}_{h} \cdot \mathbf{v}, \\
\mathscr{B}_{b}\left(p_{h}, \mathbf{v}\right) & =-\int_{\mathscr{T}_{h}} \nabla p_{h} \cdot \mathbf{v}+\int_{\mathscr{F}_{h}^{I}} \llbracket p_{h} \rrbracket \cdot(\{\mathbf{v}\}-\mathbf{b} \llbracket \mathbf{v} \rrbracket)+\int_{\mathscr{F} D} p_{h} \mathbf{v} \cdot \mathbf{n}_{F}, \\
\mathscr{S}_{b}\left(p_{h}, q\right) & =\int_{\mathscr{F}_{h} \cup \mathscr{F}_{h}^{D}} \sigma_{F} \llbracket p_{h} \rrbracket \cdot \llbracket q \rrbracket, \\
\mathscr{C}_{1}\left(p_{h}, q, p_{\Gamma, h}\right) & =\int_{\Gamma_{h}} \beta_{\Gamma} \llbracket p_{h} \rrbracket \cdot \llbracket q \rrbracket+\int_{\Gamma_{h}} \alpha_{\Gamma}\left(\left\{p_{h}\right\}-p_{\Gamma, h}\right)\{q\}, \\
\mathscr{C}_{2}\left(p_{h}, p_{\Gamma, h}, q_{\Gamma}\right) & =\int_{\Gamma_{h}} \alpha_{\Gamma}\left(p_{\Gamma, h}-\left\{p_{h}\right\}\right) q_{\Gamma},
\end{aligned}
$$

and $\mathscr{A}_{\Gamma}^{P}(\cdot, \cdot)$ and $\mathscr{L}_{\Gamma}^{P}(\cdot)$ are defined as in (34) and (36), respectively. Also note that we have $\mathscr{C}\left(\left(p_{h}, p_{\Gamma, h}\right),\left(q, q_{\Gamma}\right)\right)=$ $\mathscr{C}_{1}\left(p_{h}, q, p_{\Gamma, h}\right)+\mathscr{C}_{2}\left(p_{h}, p_{\Gamma, h}, q_{\Gamma}\right)$. For the purpose of the analysis, the bulk velocity $\mathbf{u}_{h}$ can be eliminated elementwise by introducing the lifting operator, $\mathscr{L}_{b}^{L D G}:\left[L^{1}\left(\mathscr{F}_{h}^{I} \cup \mathscr{F}_{h}^{D}\right)\right]^{d} \rightarrow \mathbf{W}_{h}^{b}$, defined by

$$
\int_{\mathscr{T}_{h}} \mathscr{L}_{b}^{L D G}(\xi) \cdot \mathbf{v}=-\int_{\mathscr{F} I}(\{\mathbf{v}\}-\mathbf{b} \llbracket \mathbf{v} \rrbracket) \cdot \xi-\int_{\mathscr{F}_{h}^{D}} \xi \cdot \mathbf{v} \quad \forall \mathbf{v} \in \mathbf{W}_{h}^{b}
$$

to obtain the following discrete formulation: find $\left(p_{h}, p_{h}^{\Gamma}\right) \in Q_{h}^{b} \times Q_{h}^{\Gamma}$ such that

$$
\mathscr{A}_{h}^{M P}\left(\left(p_{h}, p_{h}^{\Gamma}\right),\left(q, q_{\Gamma}\right)\right)=\mathscr{L}_{h}^{M P}\left(q, q_{\Gamma}\right) \quad \forall\left(q, q_{\Gamma}\right) \in Q_{h}^{b} \times Q_{h}^{\Gamma},
$$

where the superscript $M P$ stands for mixed-primal and $\mathscr{A}_{h}^{M P}:\left(Q_{h}^{b} \times Q_{h}^{\Gamma}\right) \times\left(Q_{h}^{b} \times Q_{h}^{\Gamma}\right) \rightarrow \mathbb{R}$ is defined as

$$
\mathscr{A}_{h}^{M P}\left(\left(p_{h}, p_{h}^{\Gamma}\right),\left(q, q_{\Gamma}\right)\right)=\mathscr{A}_{b}^{M}\left(p_{h}, q\right)+\mathscr{A}_{\Gamma}^{P}\left(p_{\Gamma, h}, q_{\Gamma}\right)+\mathscr{C}\left(\left(p_{h}, p_{\Gamma, h}\right),\left(q, q_{\Gamma}\right)\right) .
$$

Here, $\mathscr{L}_{h}^{M P}: Q_{h}^{b} \times Q_{h}^{\Gamma} \rightarrow \mathbb{R}$ is given by

$$
\mathscr{L}_{h}^{M P}\left(q, q_{\Gamma}\right)=\mathscr{L}_{b}^{M}(q)+\mathscr{L}_{\Gamma}^{P}\left(q_{\Gamma}\right)
$$

with

$$
\begin{aligned}
\mathscr{A}_{b}^{M}\left(p_{h}, q\right)= & \int_{\mathscr{T}_{h}} v\left(\nabla p_{h}+\mathscr{L}_{b}^{L D G}\left(\llbracket p_{h} \rrbracket\right)\right) \cdot\left(\nabla q+\mathscr{L}_{b}^{L D G}(\llbracket q \rrbracket)\right)+\int_{\mathscr{F}_{h} I^{\cup} \mathscr{F}_{h} D} \sigma_{F} \llbracket p_{h} \rrbracket \cdot \llbracket q \rrbracket \\
& +\int_{\Gamma_{h}} \beta_{\Gamma} \llbracket p_{h} \rrbracket \cdot \llbracket q \rrbracket+\int_{\Gamma_{h}} \alpha_{\Gamma}\left(\left\{p_{h}\right\}-p_{\Gamma}\right)\{q\} \\
\mathscr{L}_{b}^{M}(q)= & \int_{\mathscr{T}_{h}} f q .
\end{aligned}
$$

\subsubsection{Primal-Mixed formulation}

We next address the choice of the numerical fluxes that lead to a primal-mixed formulation, i.e. we approximate the problem in the bulk using the SIPDG method, and the problem in the fracture in mixed form, employing the LDG method. In the bulk we define the numerical fluxes $\hat{p}$ and $\hat{\mathbf{u}}$ as in (31), whereas in the fracture we define the numerical fluxes $\hat{p}_{\Gamma}=\hat{p}_{\Gamma}\left(p_{\Gamma, h}\right)$ and $\hat{\mathbf{u}}_{\Gamma}=\hat{\mathbf{u}}_{\Gamma}\left(\mathbf{u}_{\Gamma, h}, p_{\Gamma, h}\right)$ as follows 


$$
\hat{p}_{\Gamma}=\left\{\begin{array}{ll}
\left\{p_{\Gamma, h}\right\}+\mathbf{b}_{\Gamma} \cdot \llbracket p_{\Gamma, h} \rrbracket & \text { on } \mathscr{E}_{\Gamma, h}^{I}, \\
0 & \text { on } \mathscr{E} D \\
p_{\Gamma, h} & \text { on } \mathscr{E} N
\end{array} \quad \quad \hat{\mathbf{u}}_{\Gamma, h}= \begin{cases}\left\{\mathbf{u}_{\Gamma, h}\right\}-\mathbf{b}_{\Gamma} \llbracket \mathbf{u}_{\Gamma, h} \rrbracket-\sigma_{e} \llbracket p_{\Gamma, h} \rrbracket & \text { on } \mathscr{E}_{\Gamma, h}, \\
\mathbf{u}_{\Gamma, h}-\sigma_{e}\left(p_{\Gamma, h} \mathbf{n}_{e}-g_{\Gamma} \mathbf{n}_{e}\right) & \text { on } \mathscr{E}_{\Gamma, h}, \\
0 & \text { on } \mathscr{E}_{\Gamma, h}\end{cases}\right.
$$

Here, $\mathbf{b}_{\Gamma} \in\left[L^{\infty}\left(\mathrm{e}^{I}\right)\right]^{d-1}$ is a vector-valued function that is constant on each edge and it is chosen such that $\left\|\mathbf{b}_{\Gamma}\right\|_{\infty, \mathrm{e}^{I}} \lesssim 1$. This choice leads to the following primal-mixed problem: find $\left(p_{h},\left(p_{h}^{\Gamma}, \mathbf{u}_{\Gamma, h}\right)\right) \in Q_{h}^{b} \times Q_{h}^{\Gamma} \times \mathbf{W}_{h}^{\Gamma}$ such that

$$
\begin{aligned}
\mathscr{A}_{b}^{P}\left(p_{h}, q\right)+\mathscr{C}_{1}\left(\left(p_{h}, q\right), p_{\Gamma, h}\right) & =\mathscr{L}_{b}^{P}(q) & & \forall q \in Q_{h}^{b}, \\
\mathscr{M}_{\Gamma}\left(\mathbf{u}_{\Gamma, h}, \mathbf{v}_{\Gamma}\right)+\mathscr{B}_{\Gamma}\left(p_{\Gamma, h}, \mathbf{v}_{\Gamma}\right) & =0 & & \forall \mathbf{v}_{\Gamma} \in \mathbf{W}_{h}^{\Gamma}, \\
-\mathscr{B}_{\Gamma}\left(q_{\Gamma}, \mathbf{u}_{\Gamma, h}\right)+\mathscr{S}_{\Gamma}\left(p_{\Gamma, h}, q_{\Gamma}\right)+\mathscr{C}_{2}\left(p_{h},\left(p_{\Gamma, h}, q_{\Gamma}\right)\right) & =\mathscr{L}_{\Gamma}^{P}\left(q_{\Gamma}\right) & & \forall q_{\Gamma} \in Q_{h}^{\Gamma},
\end{aligned}
$$

where

$$
\begin{aligned}
& \mathscr{M}_{\Gamma}\left(\mathbf{u}_{\Gamma, h}, \mathbf{v}_{\Gamma}\right)=\int_{\Gamma_{h}}\left(v_{\Gamma}^{\tau} \ell_{\Gamma}\right)^{-1} \mathbf{u}_{\Gamma, h} \cdot \mathbf{v}_{\Gamma}, \\
& \mathscr{B}_{\Gamma}\left(p_{\Gamma, h}, \mathbf{v}_{\Gamma}\right)=-\int_{\Gamma_{h}} \mathbf{v}_{\Gamma} \cdot \nabla p_{\Gamma, h}+\int_{\mathscr{E}_{h, \Gamma}} \llbracket p_{\Gamma, h} \rrbracket \cdot\left(\left\{\mathbf{v}_{\Gamma}\right\}-\mathbf{b}_{\Gamma} \llbracket \mathbf{v}_{\Gamma} \rrbracket\right)+\int_{\mathscr{E}_{h, \Gamma}} p_{\Gamma, h} \mathbf{v}_{\Gamma} \cdot \mathbf{n}_{e}, \\
& \mathscr{S}_{b}\left(p_{\Gamma, h}, q_{\Gamma}\right)=\int_{\mathscr{E}_{\Gamma, h}} \sigma_{e} \llbracket p_{\Gamma, h} \rrbracket \cdot \llbracket q_{\Gamma} \rrbracket,
\end{aligned}
$$

and $\mathscr{A}_{b}^{P}\left(p_{h}, q\right)$ and $\mathscr{L}_{b}^{P}(q)$ are defined as in (33) and (36), respectively. The variable $\mathbf{u}_{\Gamma, h}$ can be eliminated elementwise based on employing the the lifting operator, $\mathscr{L}_{\Gamma}^{L D G}:\left[L^{1}\left(\mathscr{E}_{h} \cup \mathscr{E}_{h}\right)\right]^{d} \rightarrow \mathbf{W}_{h}^{\Gamma}$, defined by

$$
\int_{\Gamma_{h}} \mathscr{L}_{\Gamma}^{L D G}\left(\xi_{\Gamma}\right) \cdot \mathbf{v}_{\Gamma}=-\int_{\mathscr{E}_{\Gamma, h}^{I}}\left(\left\{\mathbf{v}_{\Gamma}\right\}-\mathbf{b}_{\Gamma} \llbracket \mathbf{v}_{\Gamma} \rrbracket\right) \cdot \xi_{\Gamma}-\int_{\mathscr{E}_{\Gamma, h}^{D}} \xi_{\Gamma} \cdot \mathbf{v}_{\Gamma} \quad \forall \mathbf{v}_{\Gamma} \in \mathbf{W}_{h}^{\Gamma}
$$

to obtain the following primal formulation: find $\left(p_{h}, p_{h}^{\Gamma}\right) \in Q_{h}^{b} \times Q_{h}^{\Gamma}$ such that

$$
\mathscr{A}_{h}^{P M}\left(\left(p_{h}, p_{h}^{\Gamma}\right),\left(q, q_{\Gamma}\right)\right)=\mathscr{L}_{h}^{P M}\left(q, q_{\Gamma}\right) \quad \forall\left(q, q_{\Gamma}\right) \in Q_{h}^{b} \times Q_{h}^{\Gamma},
$$

where the superscript $P M$ stands for primal-mixed and $\mathscr{A}_{h}^{P M}:\left(Q_{h}^{b} \times Q_{h}^{\Gamma}\right) \times\left(Q_{h}^{b} \times Q_{h}^{\Gamma}\right) \rightarrow \mathbb{R}$ is defined as

$$
\mathscr{A}_{h}^{P M}\left(\left(p_{h}, p_{h}^{\Gamma}\right),\left(q, q_{\Gamma}\right)\right)=\mathscr{A}_{b}^{P}\left(p_{h}, q\right)+\mathscr{A}_{\Gamma}^{M}\left(p_{\Gamma, h}, q_{\Gamma}\right)+\mathscr{C}\left(\left(p_{h}, p_{\Gamma, h}\right),\left(q, q_{\Gamma}\right)\right) .
$$

Here, $\mathscr{L}_{h}^{P M}: Q_{h}^{b} \times Q_{h}^{\Gamma} \rightarrow \mathbb{R}$ is given by

$$
\mathscr{L}_{h}^{P M}\left(q, q_{\Gamma}\right)=\mathscr{L}_{b}^{P}(q)+\mathscr{L}_{\Gamma}^{M}\left(q_{\Gamma}\right)
$$

with

$$
\begin{aligned}
& \mathscr{A}_{\Gamma}^{M}\left(p_{\Gamma, h}, q_{\Gamma}\right)=\int_{\Gamma_{h}} v_{\Gamma}^{\tau} \ell_{\Gamma}\left(\nabla p_{\Gamma, h}+\mathscr{L}_{\Gamma}^{L D G}\left(\llbracket p_{\Gamma, h} \rrbracket\right)\right) \cdot\left(\nabla q_{\Gamma}+\mathscr{L}_{\Gamma}^{L D G}\left(\llbracket q_{\Gamma} \rrbracket\right)\right) \\
& \quad+\int_{\mathscr{E}_{\Gamma, h}^{I} \cup \mathscr{E}_{\Gamma, h}^{D}} \sigma_{e} \llbracket p_{\Gamma, h} \rrbracket \cdot \llbracket q_{\Gamma} \rrbracket \\
& \mathscr{L}_{\Gamma}^{M}\left(q_{\Gamma}\right)=\int_{\Gamma_{h}} \ell_{\Gamma} f_{\Gamma} q_{\Gamma} .
\end{aligned}
$$

\subsubsection{Mixed-Mixed formulation}

Finally, if we approximate both the problem in the bulk and in the fracture with the LDG method by choosing the bulk numerical fluxes $\hat{p}=\hat{p}\left(p_{h}\right)$ and $\hat{\mathbf{u}}=\hat{\mathbf{u}}\left(\mathbf{u}_{h}, p_{h}, p_{\Gamma, h}\right)$ as in (37) and the fracture numerical fluxes $\hat{p}_{\Gamma}=\hat{p}_{\Gamma}\left(p_{\Gamma, h}\right)$ and $\hat{\mathbf{u}}_{\Gamma}=\hat{\mathbf{u}}_{\Gamma}\left(\mathbf{u}_{\Gamma, h}, p_{\Gamma, h}\right)$ as in (42), we obtain the following mixed-mixed formulation: find $\left(p_{h}, p_{\Gamma, h}\right) \in Q_{h}^{b} \times Q_{h}^{\Gamma}$ and 
$\left(\mathbf{u}_{h}, \mathbf{u}_{\Gamma, h}\right) \in \mathbf{W}_{h}^{b} \times \mathbf{W}_{h}^{\Gamma}$ such that

$$
\begin{aligned}
\mathscr{M}_{b}\left(\mathbf{u}_{h}, \mathbf{v}\right)+\mathscr{B}_{b}\left(p_{h}, \mathbf{v}\right) & =F_{b}(\mathbf{v}) & & \forall \mathbf{v} \in \mathbf{W}_{h}^{b}, \\
-\mathscr{B}_{b}\left(q, \mathbf{u}_{h}\right)+\mathscr{S}_{b}\left(p_{h}, q\right)+\mathscr{C}_{1}\left(p_{h}, q, p_{\Gamma, h}\right) & =G_{b}(q) & & \forall q \in Q_{h}^{b}, \\
\mathscr{M}_{\Gamma}\left(\mathbf{u}_{\Gamma, h}, \mathbf{v}_{\Gamma}\right)+\mathscr{B}_{\Gamma}\left(p_{\Gamma, h}, \mathbf{v}_{\Gamma}\right) & =F_{\Gamma}\left(\mathbf{v}_{\Gamma}\right) & & \forall \mathbf{v}_{\Gamma} \in \mathbf{W}_{h}^{\Gamma}, \\
-\mathscr{B}_{\Gamma}\left(q_{\Gamma}, \mathbf{u}_{\Gamma, h}\right)+\mathscr{S}_{\Gamma}\left(p_{\Gamma, h}, q_{\Gamma}\right)+\mathscr{C}_{2}\left(p_{h},\left(p_{\Gamma, h}, q_{\Gamma}\right)\right) & =G_{\Gamma}\left(q_{\Gamma}\right) & & \forall q_{\Gamma} \in Q_{h}^{\Gamma} .
\end{aligned}
$$

Again, based on employing the definition of the lifting operators (39) and (44), the bulk and fracture velocities can be eliminated, to yield the following equivalent formulation: find $\left(p_{h}, p_{\Gamma, h}\right) \in Q_{h}^{b} \times Q_{h}^{\Gamma}$ such that

$$
\mathscr{A}_{h}^{M M}\left(\left(p_{h}, p_{h}^{\Gamma}\right),\left(q, q_{\Gamma}\right)\right)=\mathscr{L}_{h}^{M M}\left(q, q_{\Gamma}\right) \quad \forall\left(q, q_{\Gamma}\right) \in Q_{h}^{b} \times Q_{h}^{\Gamma},
$$

where the superscript $M M$ stands for mixed-mixed and $\mathscr{A}_{h}^{M M}:\left(Q_{h}^{b} \times Q_{h}^{\Gamma}\right) \times\left(Q_{h}^{b} \times Q_{h}^{\Gamma}\right) \rightarrow \mathbb{R}$ is defined as

$$
\mathscr{A}_{h}^{M M}\left(\left(p_{h}, p_{h}^{\Gamma}\right),\left(q, q_{\Gamma}\right)\right)=\mathscr{A}_{b}^{M}\left(p_{h}, q\right)+\mathscr{A}_{\Gamma}^{M}\left(p_{\Gamma, h}, q_{\Gamma}\right)+\mathscr{C}\left(\left(p_{h}, p_{\Gamma, h}\right),\left(q, q_{\Gamma}\right)\right),
$$

and $\mathscr{L}_{h}^{M M}: Q_{h}^{b} \times Q_{h}^{\Gamma} \rightarrow \mathbb{R}$ is given by

$$
\mathscr{L}_{h}^{M M}\left(q, q_{\Gamma}\right)=\mathscr{L}_{b}^{M}(q)+\mathscr{L}_{\Gamma}^{M}\left(q_{\Gamma}\right)
$$

\subsection{Well-posedness and error estimates}

In this section, we recall the main results that ensure that the primal-primal (PP) (32), mixed-primal (MP) (40), primal-mixed (PM) (45) and mixed-mixed (MM)(48) formulations are well-posed. We recall that, for the analysis, we assume the permeability tensors $v$ and $v_{\Gamma}^{\tau}$ to be piecewise constant and that we employ the following notation $\bar{v}_{E}=\left|\sqrt{\left.v\right|_{E}}\right|_{2}^{2}$ and $\bar{v}_{F}^{\tau}=\left|\sqrt{\left.v_{\Gamma}^{\tau}\right|_{F}}\right|_{2}^{2}$, where $|\cdot|_{2}$ denotes the $l_{2}$-norm. First, we give an appropriate definition of the discontinuity penalization parameters, so that we can work in a polytopic framework. Taking as a reference [67, 64, $13,63,66]$, we give the following two definitions for the bulk and fracture penalty functions.

Definition 5. The penalization parameter $\sigma: \mathscr{F}_{h} \backslash \Gamma_{h} \rightarrow \mathbb{R}^{+}$for the bulk problem is defined facewise as

$$
\sigma(\mathbf{x})=\sigma_{0} \begin{cases}\max _{E \in\left\{E^{+}, E^{-}\right\}} \frac{\bar{v}_{E} p_{E}^{2}}{h_{E}} & \text { if } \mathbf{x} \subset F \in \mathscr{F}_{h}^{I}, \bar{F}=\partial \bar{E}^{+} \cap \partial \bar{E}^{-}, \\ \frac{\bar{v}_{E} p_{E}^{2}}{h_{E}} & \text { if } \mathbf{x} \subset F \in \mathscr{F}_{h}^{D}, \bar{F}=\partial \bar{E} \cap \partial \bar{\Omega},\end{cases}
$$

with $\sigma_{0}>0$ independent of $p_{E},|E|$, and $|F|$. Analogously, the penalization parameter $\sigma_{\Gamma}: \mathrm{e} \rightarrow \mathbb{R}^{+}$for the fracture problem is defined edgewise as

$$
\sigma_{\Gamma}(\mathbf{x})=\sigma_{0, \Gamma} \begin{cases}\max _{F \in\left\{F^{+}, F^{-}\right\}} \frac{\bar{v}_{F}^{\tau} p_{F}^{2}}{h_{F}} & \text { if } \mathbf{x} \subset e \in \mathrm{e}^{I}, \bar{e}=\partial \bar{F}^{+} \cap \partial \bar{F}^{-}, \\ \frac{\bar{v}_{F}^{\tau} p_{F}^{2}}{h_{F}}, & \text { if } \mathbf{x} \subset e \in \mathrm{e}^{D}, \bar{e}=\partial \bar{F} \cap \partial \bar{\Gamma},\end{cases}
$$

with $\sigma_{0, \Gamma}>0$ independent of $p_{F},|F|$, and $|e|$.

Writing $\tilde{Q}^{b}=\left\{q=\left(q_{1}, q_{2}\right) \in H^{1}\left(\Omega_{1}\right) \times H^{1}\left(\Omega_{2}\right)\right\} \cap H^{2}\left(\mathscr{T}_{h}\right)$ and $\tilde{Q}^{\Gamma}=H^{1}(\Gamma) \cap H^{2}\left(\Gamma_{h}\right)$, we introduce the spaces $Q^{b}(h)=Q_{h}^{b}+\tilde{Q}^{b}$ and $Q^{\Gamma}(h)=Q_{h}^{\Gamma}+\tilde{Q}^{\Gamma}$ endowed with the energy norm

$$
\left\|\left(q, q_{\Gamma}\right) \mid\right\|^{2}=\|q\|_{b, D G}^{2}+\left\|q_{\Gamma}\right\|_{\Gamma, D G}^{2}+\left\|\left(q, q_{\Gamma}\right)\right\|_{\mathscr{C}}^{2},
$$

where 


$$
\begin{aligned}
\|q\|_{b, D G}^{2} & =\left\|v^{1 / 2} \nabla q\right\|_{0, \mathscr{F}_{h}}^{2}+\left\|\sigma_{F}^{1 / 2} \llbracket q \rrbracket\right\|_{0, \mathscr{F}_{h}^{I} \cup \mathscr{F}_{h}^{D},}^{2} \\
\left\|q_{\Gamma}\right\|_{\Gamma, D G}^{2} & =\left\|\left(v_{\Gamma}^{\tau} \ell_{\Gamma}\right)^{1 / 2} \nabla q_{\Gamma}\right\|_{0, \Gamma_{h}}^{2}+\left\|\sigma_{e}^{1 / 2} \llbracket q_{\Gamma} \rrbracket\right\|_{0, \mathrm{e}^{l} \cup \mathrm{e} D}^{2}, \\
\left\|\left(q, q_{\Gamma}\right)\right\|_{\mathscr{C}}^{2} & =\left\|\beta_{\Gamma}^{1 / 2} \llbracket q \rrbracket\right\|_{0, \Gamma_{h}}^{2}+\left\|\alpha_{\Gamma}^{1 / 2}\left(\{q\}-q_{\Gamma}\right)\right\|_{0, \Gamma_{h}}^{2} .
\end{aligned}
$$

We remark that all the bilinear forms $\mathscr{A}_{h}^{* *}(\cdot, \cdot), * * \in\{P P, M P, M M, P M\}$, defined in Section 5.2 are also well-defined on the extended space $Q^{b}(h) \times Q^{\Gamma}(h)$. We now recall the following result, and refer to [16] for the proof.

Lemma 7. The following bounds hold

$$
\begin{aligned}
& \mathscr{A}_{b}^{P}(q, q) \gtrsim\|q\|_{b, D G}^{2} \\
& \forall q \in Q_{h}^{b}, \\
& \mathscr{A}_{b}^{P}(p, q) \lesssim\|p\|_{b, D G}\|q\|_{b, D G} \\
& \mathscr{A}_{\Gamma}^{P}\left(q_{\Gamma}, q_{\Gamma}\right) \gtrsim\left\|q_{\Gamma}\right\|_{\Gamma, D G}^{2} \\
& \forall p, q \in Q^{b}(h), \\
& \mathscr{A}_{\Gamma}^{P}\left(p_{\Gamma}, q_{\Gamma}\right) \lesssim\left\|p_{\Gamma}\right\|_{\Gamma, D G}\left\|q_{\Gamma}\right\|_{\Gamma, D G} \\
& \forall q_{\Gamma} \in Q_{h}^{\Gamma}, \\
& \mathscr{A}_{b}^{M}(q, q) \gtrsim\|q\|_{b, D G}^{2} \\
& \mathscr{A}_{b}^{M}(p, q) \lesssim\|p\|_{b, D G}\|q\|_{b, D G} \\
& \mathscr{A}_{\Gamma}^{M}\left(q_{\Gamma}, q_{\Gamma}\right) \gtrsim\left\|q_{\Gamma}\right\|_{\Gamma, D G}^{2} \\
& \mathscr{A}_{\Gamma}^{M}\left(p_{\Gamma}, q_{\Gamma}\right) \lesssim\left\|p_{\Gamma}\right\|_{\Gamma, D G}\left\|q_{\Gamma}\right\|_{\Gamma, D G} \\
& \forall p_{\Gamma}, q_{\Gamma} \in Q^{\Gamma}(h), \\
& \forall q \in Q_{h}^{b}, \\
& \forall p, q \in Q^{b}(h), \\
& \forall q_{\Gamma} \in Q_{h}^{\Gamma}, \\
& \forall p_{\Gamma}, q_{\Gamma} \in Q^{\Gamma}(h) .
\end{aligned}
$$

The first and third estimates hold provided that $\sigma_{0}$ and $\sigma_{0, \Gamma}$ are chosen sufficiently large.

Employing Lemma 7, we can easily prove the well-posedness of all of our discrete problems, as stated in the following proposition.

Proposition 3. Let the penalization parameters $\sigma$ for the problem in the bulk and in the fracture be defined as in (49) and (50), respectively, and suppose that for the primal formulations $\sigma_{0}$ and $\sigma_{0, \Gamma}$ are chosen sufficiently large. Then, all the formulations (32), (40), (45) and (48) are well-posed.

Next we prove error bounds in the discrete energy norm (51). To this end, for each subdomain $\Omega_{i}, i=1,2$, we denote by $\mathscr{E}_{i}$ the classical continuous extension operator (cf. [136]) $\mathscr{E}_{i}: H^{s}\left(\Omega_{i}\right) \rightarrow H^{s}\left(\mathbb{R}^{d}\right.$ ), for $s \in \mathbb{N}_{0}$. Similarly, we denote by $\mathscr{E}_{\Gamma}$ the continuous extension operator $\mathscr{E}_{\Gamma}: H^{s}(\Gamma) \rightarrow H^{s}\left(\mathbb{R}^{d-1}\right)$, for $s \in \mathbb{N}_{0}$. We then make the following regularity assumptions for the analytical solution $\left(p, p_{\Gamma}\right)$ of problem (30).

Assumption 6 Let $\mathscr{T}_{\#}=\left\{T_{E}\right\}$ and $\mathscr{F}_{\#}=\left\{T_{F}\right\}$ denote the associated coverings of $\Omega$ and $\Gamma$, respectively, cf. Definition 3. We assume that the analytical solution $\left(p, p_{\Gamma}\right)$ is such that:

AlFor every $E \in \mathscr{T}_{h}$, if $E \subset \Omega_{i}$, we have $\left.\mathscr{E}_{i} p_{i}\right|_{T_{E}} \in H^{r_{E}}\left(T_{E}\right)$, where $r_{E} \geq 1+d / 2$ and $T_{E} \in \mathscr{T}_{\#}$, with $E \subset T_{E}$; A2.For every $F \in \Gamma_{h}$, we have $\left.\mathscr{E}_{\Gamma} p_{\Gamma}\right|_{T_{F}} \in H^{r_{F}}\left(T_{F}\right)$, where $r_{F} \geq 1+(d-1) / 2$ and $T_{F} \in \mathscr{F}_{\#}$, with $F \subset T_{F}$.

Assumption 7 We assume that the normal components of the exact fluxes $v \nabla p$ and $\ell_{\Gamma} v_{\Gamma}^{\tau} \nabla p_{\Gamma}$ are continuous across mesh interfaces, that is $\llbracket v \nabla p \rrbracket=0$ on $\mathscr{F}_{h}^{I}$ and $\llbracket \ell_{\Gamma} v_{\Gamma}^{\tau} \nabla p_{\Gamma} \rrbracket=0$ on $\mathrm{e}^{I}$.

From Proposition 3 and Strang's second lemma, the following abstract error bound follows directly.

Lemma 8. Let the hypotheses of Proposition 3 be satisfied. Then, for all the discrete formulations presented in Section 5.2, the following abstract error bound holds

$$
\left\|\left|\left(p, p_{\Gamma}\right)-\left(p_{h}, p_{\Gamma, h}\right)\right|\right\|\left|\inf _{\left(q, q_{\Gamma}\right) \in Q_{h}^{b} \times Q_{h}^{\Gamma}}\right|||\left(p, p_{\Gamma}\right)-\left(q, q_{\Gamma}\right)|\||+\sup _{\left(w, w_{\Gamma}\right) \in Q_{h}^{b} \times Q_{h}^{\Gamma}} \frac{\left|\mathscr{R}_{h}^{* *}\left(\left(p, p_{\Gamma}\right),\left(w, w_{\Gamma}\right)\right)\right|}{\left\|\left|\left(w, w_{\Gamma}\right)\right|\right\|}
$$

where the residual $\mathscr{R}_{h}^{* *}$ is defined as

$$
\mathscr{R}_{h}^{* *}\left(\left(p, p_{\Gamma}\right),\left(w, w_{\Gamma}\right)\right)=\mathscr{A}_{h}^{* *}\left(\left(p, p_{\Gamma}\right),\left(w, w_{\Gamma}\right)\right)-\mathscr{L}_{h}^{* *}\left(w, w_{\Gamma}\right),
$$

with $* * \in\{P P, M P, M M, P M\}$. 
We now recall the following result that provides a bound on the residuals stemming from formulations (32), (40), (45) and (48).

Lemma 9. [16, Lemma 5.6, Lemma 5.7] Let $\left(p, p_{\Gamma}\right)$ be the analytical solution of problem (30) satisfying the regularity Assumptions 6 and 7. Then, for every $w \in Q^{b}(h)$ and $w_{\Gamma} \in Q^{\Gamma}(h)$, we have that

$$
\begin{aligned}
\left|\mathscr{R}_{b}^{P}(p, w)\right|^{2} & \lesssim \sum_{E \in \mathscr{T}_{h}} \frac{h_{E}^{2\left(s_{E}-1\right)}}{p_{E}^{2\left(r_{E}-1\right)}}\|\mathscr{E} p\|_{H^{r_{E}\left(T_{E}\right)}}^{2}\left[\bar{v}_{E}^{2}\right] \cdot\|w\|_{b, D G}^{2}, \\
\left|\mathscr{R}_{\Gamma}^{P}\left(p_{\Gamma}, w_{\Gamma}\right)\right|^{2} & \lesssim \sum_{F \in \Gamma_{h}} \frac{h_{F}^{2\left(s_{F}-1\right)}}{p_{F}^{2\left(r_{F}-1\right)}}\left\|\mathscr{E} p_{\Gamma}\right\|_{H^{r_{F}\left(T_{F}\right)}}^{2}\left[\left(\bar{v}_{F}^{\tau} \ell_{\Gamma}\right)^{2}\right] \cdot\left\|w_{\Gamma}\right\|_{\Gamma, D G}^{2}, \\
\left|\mathscr{R}_{b}^{M}(p, w)\right|^{2} & \lesssim \sum_{E \in \mathscr{T}_{h}} \frac{h_{E}^{2\left(s_{E}-1\right)}}{p_{E}^{2\left(r_{E}-1\right)}}\|\mathscr{E} p\|_{H^{r_{E}\left(T_{E}\right)}}^{2}\left[\bar{v}_{E}^{2}\right] \cdot\|w\|_{b, D G}^{2}, \\
\left|\mathscr{R}_{\Gamma}^{M}\left(p_{\Gamma}, w_{\Gamma}\right)\right|^{2} & \left.\lesssim \sum_{F \in \Gamma_{h}} \frac{h_{F}^{2\left(s_{F}-1\right)}}{p_{F}^{2\left(r_{F}-1\right)}}\left\|\mathscr{E} p_{\Gamma}\right\|_{H^{r_{F}\left(T_{F}\right)}}^{2}\left[\bar{v}_{F}^{\tau} \ell_{\Gamma}\right)^{2}\right] \cdot\left\|w_{\Gamma}\right\|_{\Gamma, D G}^{2} .
\end{aligned}
$$

The above bounds, together with the observation that, for all the cases, the residual can always be split into two contributions: one involving the approximation of the problem in the bulk and one involving the approximation of the problem in the fracture, i.e.,

$$
\mathscr{R}_{h}^{* *}\left(\left(p, p_{\Gamma}\right),\left(w, w_{\Gamma}\right)\right)=\mathscr{R}_{b}^{*}(p, w)+\mathscr{R}_{\Gamma}^{*}\left(p_{\Gamma}, w_{\Gamma}\right),
$$

are the key ingredients required to derive main result of this section.

Theorem 3. Let $\mathscr{T}_{\#}=\left\{T_{E}\right\}$ and $\mathscr{F}_{\#}=\left\{T_{F}\right\}$ denote the associated coverings of $\Omega$ and $\Gamma$, respectively, consisting of shape-regular simplices as in Definition 3, satisfying Assumption 4. Let $\left(p, p_{\Gamma}\right)$ be the solution of problem (30) and $\left(p_{h}, p_{\Gamma, h}\right) \in Q_{h}^{b} \times Q_{h}^{\Gamma}$ be its approximation obtained with the method PP, MP, MM or PM, with the penalization parameters given by (49) and (50) and $\sigma_{0}$ and $\sigma_{0, \Gamma}$ sufficiently large for the primal formulations. Moreover, suppose that the analytical solution $\left(p, p_{\Gamma}\right)$ satisfies the regularity Assumptions 6 and 7. Then, the following error bound holds

$$
\left\|\left|\left(p, p_{\Gamma}\right)-\left(p_{h}, p_{\Gamma, h}\right)\left\|\left.\right|^{2} \lesssim \sum_{E \in \mathscr{T}_{h}} \frac{h_{E}^{2\left(s_{E}-1\right)}}{p_{E}^{2\left(r_{E}-1\right)}} G_{E}^{*}\left(\bar{v}_{E}\right)\right\| \mathscr{E} p\left\|_{H^{r_{E}\left(T_{E}\right)}}^{2}+\sum_{F \in \Gamma_{h}} \frac{h_{F}^{2\left(s_{F}-1\right)}}{p_{F}^{2\left(r_{F}-1\right)}} G_{F}^{*}\left(\bar{v}_{F}^{\tau}\right)\right\| \mathscr{E}_{\Gamma} p_{\Gamma} \|_{H^{r_{F}}\left(T_{F}\right)}^{2},\right.\right.
$$

where $\mathscr{E} p$ is to be interpreted as $\mathscr{E}_{1} p_{1}$ when $E \subset \Omega_{1}$ or as $\mathscr{E}_{2} p_{2}$ when $E \subset \Omega_{2}$. Here, $s_{E}=\min \left(p_{E}+1, r_{E}\right), s_{F}=$ $\min \left(p_{F}+1, r_{F}\right)$, and the constants satisfy

$$
G_{E}^{P}\left(\bar{v}_{E}\right) \lesssim \bar{v}_{E} \quad G_{F}^{P}\left(\bar{v}_{F}^{\tau}\right) \lesssim \bar{v}_{F}^{\tau}, \quad G_{E}^{M}\left(\bar{v}_{E}\right) \lesssim \bar{v}_{E} \quad G_{F}^{M}\left(\bar{v}_{F}^{\tau}\right) \lesssim \bar{v}_{F}^{\tau} \ell_{\Gamma} .
$$

Proof. From Lemma 8 we deduce that the error satisfies the following abstract bound

$$
\left\|\left|\left(p, p_{\Gamma}\right)-\left(p_{h}, p_{\Gamma, h}\right)\right|\right\| \mid \lesssim \underbrace{\inf _{\left(q, q_{\Gamma}\right) \in Q_{h}^{b} \times Q_{h}^{\Gamma}}\left|\|\left(p, p_{\Gamma}\right)-\left(q, q_{\Gamma}\right)\right|||}_{I}+\underbrace{\sup _{\left(w, w_{\Gamma}\right) \in Q_{h}^{b} \times Q_{h}^{\Gamma}} \frac{\left|\mathscr{R}_{h}\left(\left(p, p_{\Gamma}\right),\left(w, w_{\Gamma}\right)\right)\right|}{\|||\left(w, w_{\Gamma}\right)|| \mid}}_{I I} .
$$

For the term $I$, exploiting the approximation results stated in Lemma 2, we obtain

$$
I \lesssim \sum_{E \in \mathscr{T} h} \bar{v}_{E} \frac{h_{E}^{2\left(s_{E}-1\right)}}{p_{E}^{2\left(r_{E}-1\right)}}\|\mathscr{E} p\|_{H^{r} E\left(T_{E}\right)}^{2}+\sum_{F \in \Gamma_{h}} \bar{v}_{F}^{\tau} \ell_{\Gamma} \frac{h_{F}^{2\left(s_{F}-1\right)}}{p_{F}^{2\left(r_{F}-1\right)}}\left\|\mathscr{E} p_{\Gamma}\right\|_{H^{r_{F}\left(T_{F}\right)}}^{2}
$$

The statement of the theorem follows from (53), together with the bound on Term II deriving from what observed in (52) and Lemma 9.

If the hypotheses of Theorem 3 hold, we can also derive error estimates for the velocities $\mathbf{u}$ and $\mathbf{u}_{\Gamma}$ for the mixedprimal, primal-mixed, and mixed-mixed formulations. More precisely, if $\left(\mathbf{u}, \mathbf{u}_{\Gamma}\right) \in \mathbf{W}$ and $\left(p, p_{\Gamma}\right) \in M$ is the solution of problem (30), then, if $\left(\left(p_{h}, \mathbf{u}_{h}\right), p_{\Gamma, h}\right) \in Q_{h}^{b} \times \mathbf{W}_{h}^{b} \times Q_{h}^{\Gamma}$ is the approximation obtained with the mixed-primal method (38), we have that 


$$
\left\|\mathbf{u}-\mathbf{u}_{h}\right\|_{0, \mathscr{T}_{h}}^{2} \lesssim \sum_{E \in \mathscr{T}_{h}} \frac{h_{E}^{2\left(s_{E}-1\right)}}{p_{E}^{2\left(r_{E}-1\right)}} G_{E}^{M}\|\mathscr{E} p\|_{H^{r} E\left(T_{E}\right)}^{2}+\sum_{F \in \Gamma_{h}} \frac{h_{F}^{2\left(s_{F}-1\right)}}{p_{F}^{2\left(r_{F}-1\right)}} G_{F}^{P}\left\|\mathscr{E}_{\Gamma} p_{\Gamma}\right\|_{H^{r} F\left(T_{F}\right)}^{2}
$$

Analogously, if $\left(p_{h},\left(p_{\Gamma, h}, \mathbf{u}_{\Gamma, h}\right)\right) \in Q_{h}^{b} \times Q_{h}^{\Gamma} \times \mathbf{W}_{h}^{\Gamma}$ is the approximation computed with the primal-mixed method (43), we deduce that

$$
\left\|\mathbf{u}_{\Gamma}-\mathbf{u}_{\Gamma, h}\right\|_{0, \Gamma_{h}}^{2} \lesssim \sum_{E \in \mathscr{T}_{h}} \frac{h_{E}^{2\left(s_{E}-1\right)}}{p_{E}^{2\left(r_{E}-1\right)}} G_{E}^{P}\|\mathscr{E} p\|_{H^{r} E\left(T_{E}\right)}^{2}+\sum_{F \in \Gamma_{h}} \frac{h_{F}^{2\left(s_{F}-1\right)}}{p_{F}^{2\left(r_{F}-1\right)}} G_{F}^{M}\left\|\mathscr{E}_{\Gamma} p_{\Gamma}\right\|_{H^{r}\left(T_{F}\right)}^{2}
$$

Finally, if $\left(\left(p_{h}, \mathbf{u}_{h}\right),\left(p_{\Gamma, h}, \mathbf{u}_{\Gamma, h}\right)\right) \in Q_{h}^{b} \times \mathbf{W}_{h}^{b} \times Q_{h}^{\Gamma} \times \mathbf{W}_{h}^{\Gamma}$ is the approximation obtained with the mixed-mixed method (47), then the following bound holds

$$
\left\|\mathbf{u}-\mathbf{u}_{h}\right\|_{0, \mathscr{T}_{h}}^{2}+\left\|\mathbf{u}_{\Gamma}-\mathbf{u}_{\Gamma, h}\right\|_{0, \Gamma_{h}}^{2} \lesssim \sum_{E \in \mathscr{T}_{h}} \frac{h_{E}^{2\left(s_{E}-1\right)}}{p_{E}^{2\left(r_{E}-1\right)}} G_{E}^{M}\|\mathscr{E} p\|_{H^{r} E\left(T_{E}\right)}^{2}+\sum_{F \in \Gamma_{h}} \frac{h_{F}^{2\left(s_{F}-1\right)}}{p_{F}^{2\left(r_{F}-1\right)}} G_{F}^{M}\left\|\mathscr{E}_{\Gamma} p_{\Gamma}\right\|_{H^{r} F\left(T_{F}\right)}^{2} .
$$

Here, the constants $G_{E}^{M}, G_{F}^{P}, G_{E}^{P}$ and $G_{F}^{M}$ are defined as in Theorem 3. We refer to [16] for further details.

\subsection{Numerical results}

In this section we present three sets of two-dimensional numerical experiments employing the paradigmatic primalprimal and mixed-primal settings. With the first set of experiments we aim to validate the theoretical convergence results of Section 5.3, by considering a test case with known analytical solution. With the second and third sets of experiments, we assess the capability of the method of handling more complicated geometries, namely networks of partially immersed fractures and networks of intersecting fractures. All the numerical tests have been implemented in MATLAB ${ }^{\circledR}$ and the polygonal meshes conforming to the fractures have been obtained by suitably modifying the code PolyMesher [142].

\subsubsection{Example 1: Problem with a known analytical solution}

We consider the domain $\Omega=(0,1)^{2}$ and the fracture $\Gamma=\{(x, y) \in \Omega: x=0.5\}$. Following [73, 14], we select the analytical solution in the bulk and the fracture as follows

$$
p=\left\{\begin{array}{ll}
\sin (4 x) \cos (\pi y) & \text { if } x<0.5, \\
\cos (4 x) \cos (\pi y) & \text { if } x>0.5,
\end{array} \quad p_{\Gamma}=\xi[\cos (2)+\sin (2)] \cos (\pi y),\right.
$$

so that they satisfy the coupling conditions (29i)-(29j) with $v=\mathbf{I}$, provided that $\beta_{\Gamma}=2$, that is $v_{\Gamma}^{n} / \ell_{\Gamma}=4$. In particular, here we choose the tangential and normal components of the permeability tensor in the fracture as $v_{\Gamma}^{\tau}=10^{2}$ and $v_{\Gamma}^{n}=$ $4 \cdot 10^{-2}$, respectively, and the fracture thickness $\ell_{\Gamma}=10^{-2}$. Moreover, in the experiments we set $\xi=\frac{3}{4}$. We impose

Fig. 18 Example 1 (Section 5.4.1). Three refinements of the polygonal mesh grid conforming to the fracture.
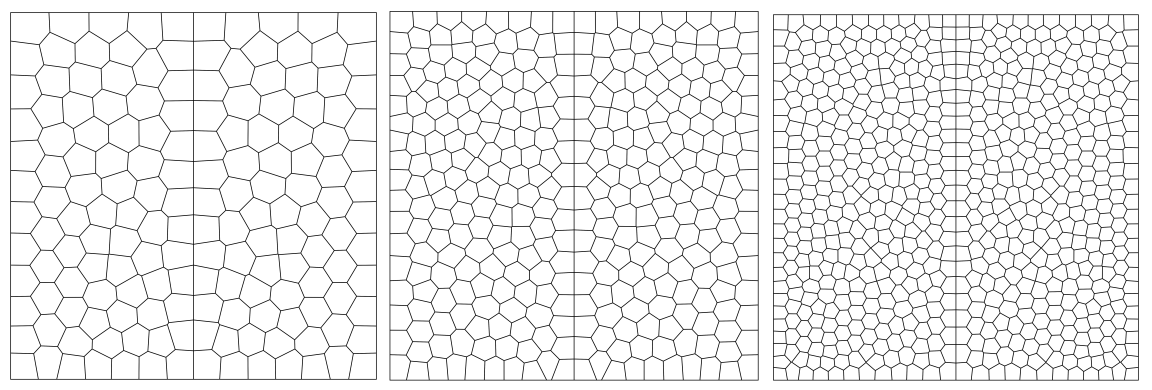


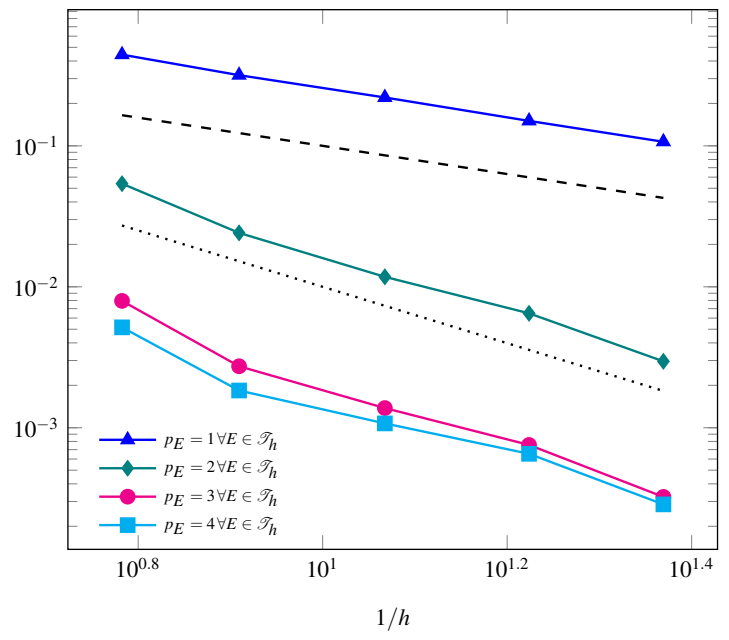

(a) Primal-primal

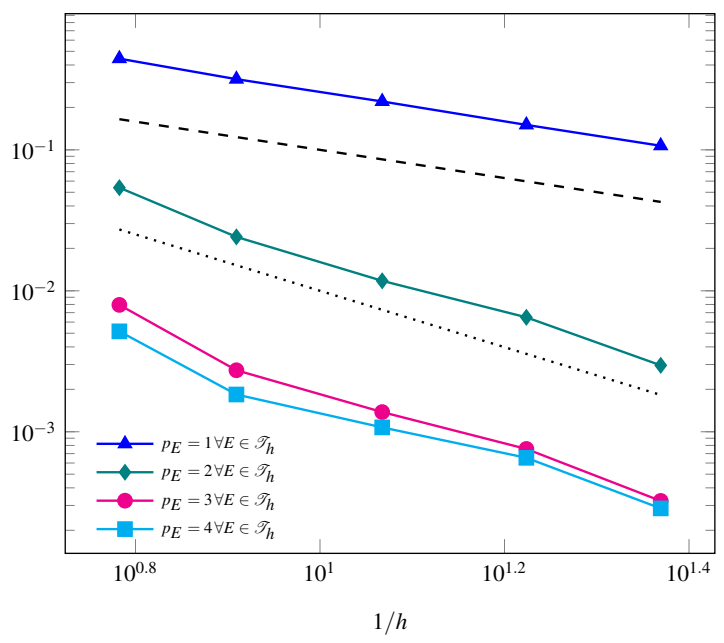

(b) Mixed-primal

Fig. 19 Example 1 (Section 5.4.1). Computed errors as a function of $1 / h(\log \log$ scale) and expected convergence rates for uniform bulk polynomial degrees $p_{E}=1,2,3,4$ for all $E \in \mathscr{T}_{h}$ and fixed uniform fracture polynomial degree $p_{F}=2$ for all $F \in \Gamma_{h}$. Case (PP) on the left and (MP) on the right.

Dirichlet boundary conditions on the whole $\partial \Omega$ and also on $\partial \Gamma$. Finally the source terms are chosen accordingly as

$$
f=\left\{\begin{array}{ll}
\sin (4 x) \cos (\pi y)\left(16+\pi^{2}\right) & \text { if } x<0.5, \\
\cos (4 x) \cos (\pi y)\left(16+\pi^{2}\right) & \text { if } x>0.5,
\end{array} \quad f_{\Gamma}=\cos (\pi y)[\cos (2)+\sin (2)]\left(\xi v_{\Gamma}^{\tau} \pi^{2}+\frac{4}{\ell_{\Gamma}}\right) .\right.
$$

In Figure 18, we show three levels of refinement of the polygonal mesh conforming to the fracture employed in the computations. In order to test the $h$-convergence properties of our methods, thus validating the error estimate for the energy norm stated in Theorem 3, we compute the quantity ||$p-p_{h}\left\|_{1, \mathscr{T}_{h}}+|| p_{\Gamma}-p_{\Gamma, h}\right\|_{1, \Gamma_{h}}$. The plots in Figure 19 show the computed errors as a function of the inverse of the mesh size $h$ (loglog scale), together with the expected convergence rates. In particular, Figure 19(a) shows the results obtained with the primal-primal approximation, while Figure 19(b) shows the analogous results for the mixed-primal method. Each plot consists of four lines: every line shows the behaviour of the energy norm of the error for a different polynomial degree in the bulk (we consider uniform polynomial degrees $p_{E}=1,2,3,4$ for all $E \in \mathscr{T}_{h}$ ). For the fracture problem we always choose a uniform quadratic polynomial degree, i.e., $k_{F}=2$ for all $F \in \Gamma_{h}$. For both the (PP) and (MP) method the theoretical convergence rates are clearly obtained, coinciding with $\min \left(p_{E}, p_{F}\right)$. In particular, the convergence rate is equal to 1 in the linear case, i.e., when $p_{E}=1$ for all $E \in \mathscr{T}_{h}$, and it is equal to 2 in all the other cases, since the approximation of the fracture problem is always quadratic. Note also that the (PP) and (MP) methods achieve the same level of accuracy.

\subsubsection{Example 2: Immersed fractures}

We now investigate the capability of our discretization methods to deal with immersed fractures. To this end, we take as a reference [4], where the mathematical model [118] is extended to fully immersed fractures. In particular, we supplement equations (29) with a condition prescribing the behaviour of the fluid at the fracture tips immersed in the porous medium. As in [4], we impose that $v_{\Gamma}^{\tau} \nabla_{\tau} p_{\Gamma} \cdot \tau=0$ on $\partial \Gamma \backslash \partial \Omega$, i.e., that the mass transfer across the immersed tips can be neglected.

We employ again the paradigmatic primal-primal and mixed-primal approximation schemes to reproduce some numerical experiments already proposed in [4]. We consider the computational domain $\Omega=(0,1)^{2}$ cut by four partially immersed fractures, namely $\Gamma_{1}=\left\{(x, y) \in(0,1)^{2}: x \geq 0.3, y=0.2\right\}, \Gamma_{2}=\left\{(x, y) \in(0,1)^{2}: x \leq 0.7, y=0.4\right\}, \Gamma_{3}=$ $\left\{(x, y) \in(0,1)^{2}: x \geq 0.3, y=0.6\right\}, \Gamma_{4}=\left\{(x, y) \in(0,1)^{2}: x \leq 0.7, y=0.8\right\}$. The fractures $\Gamma_{2}$ and $\Gamma_{4}$ are impermeable $\left(v_{\Gamma}^{\tau}=v_{\Gamma}^{n}=10^{-2}\right)$, while $\Gamma_{1}$ and $\Gamma_{3}$ are partially permeable $\left(v_{\Gamma}^{n}=10^{-2}, v_{\Gamma}^{\tau} \in\{100,1\}\right)$. With the aim of investigating the dependence of the flow on the physical properties of the fractures, we consider two different configurations (A 
Fig. 20 Example 2 (Section 5.4.2). Immersed fractures: configurations and boundary condition for the test cases $\mathrm{A}$ and $\mathrm{B}$.

Bulk pressure field and streamlines
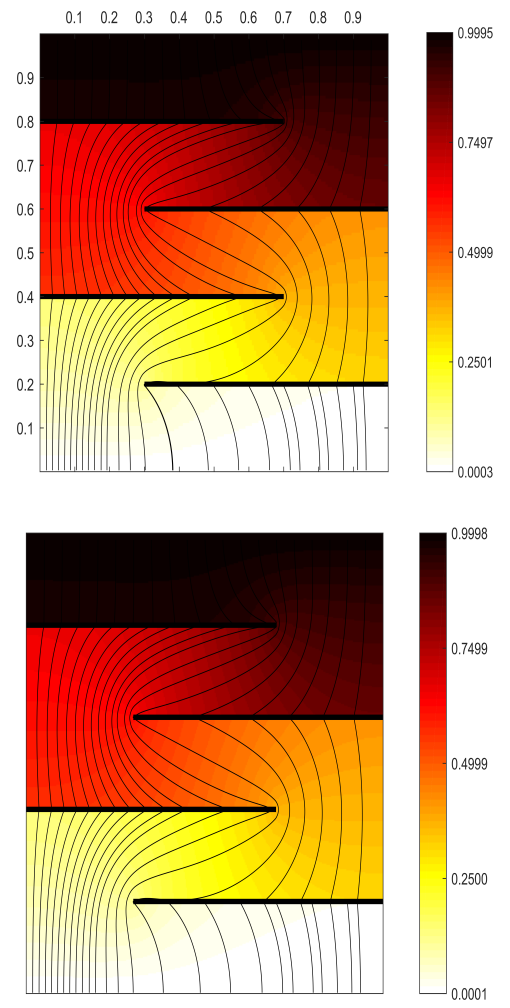

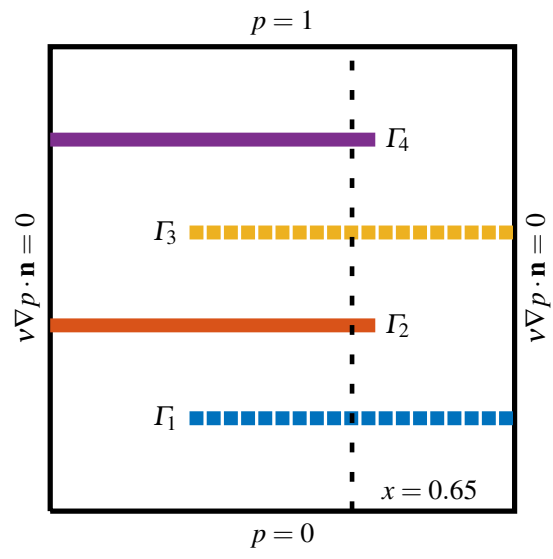

(a) Configuration A: $v_{\Gamma}^{\tau}=100$ on $\Gamma_{1}, \Gamma_{3}$
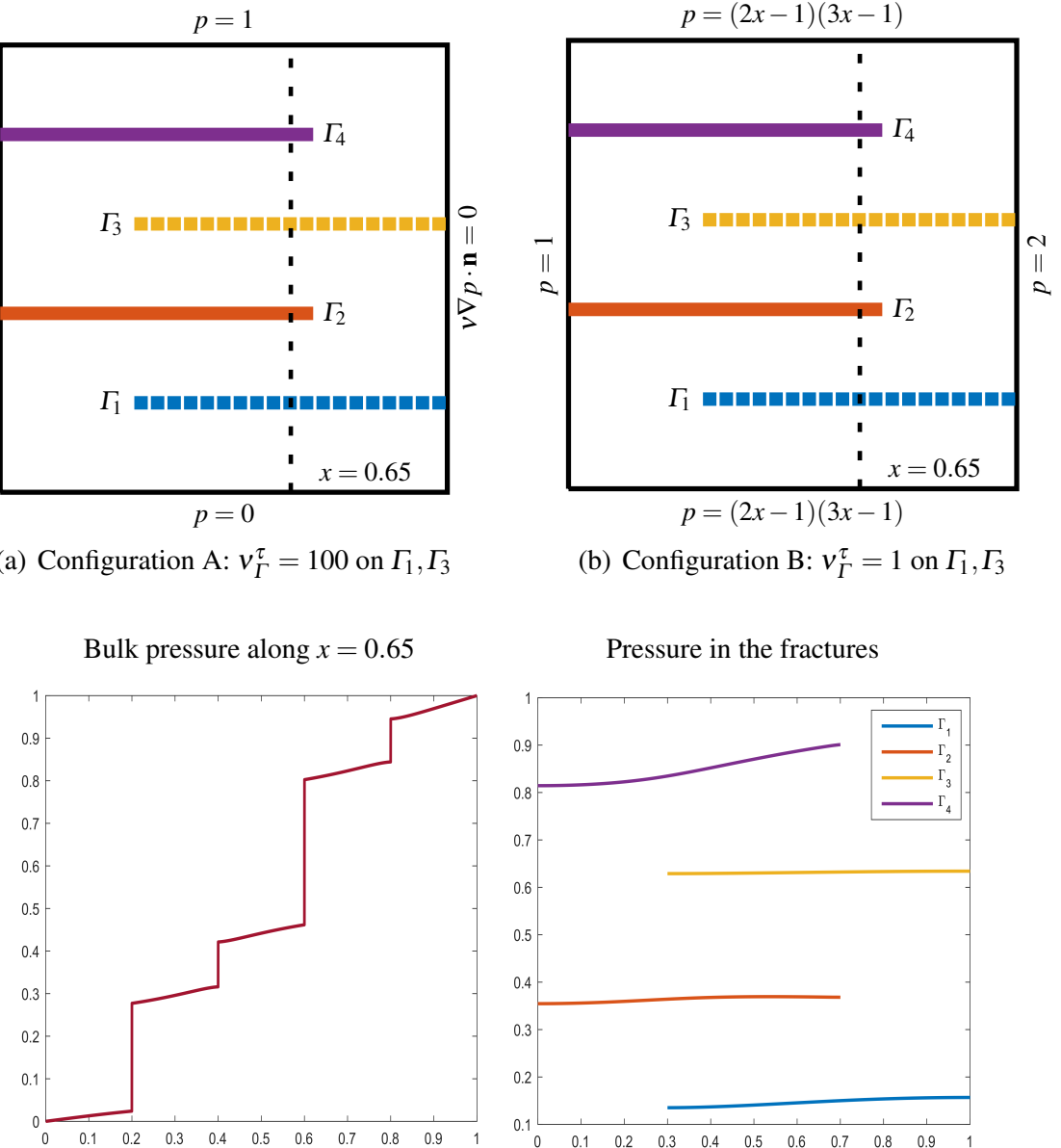

(b) Configuration B: $v_{\Gamma}^{\tau}=1$ on $\Gamma_{1}, \Gamma_{3}$

Pressure in the fractures
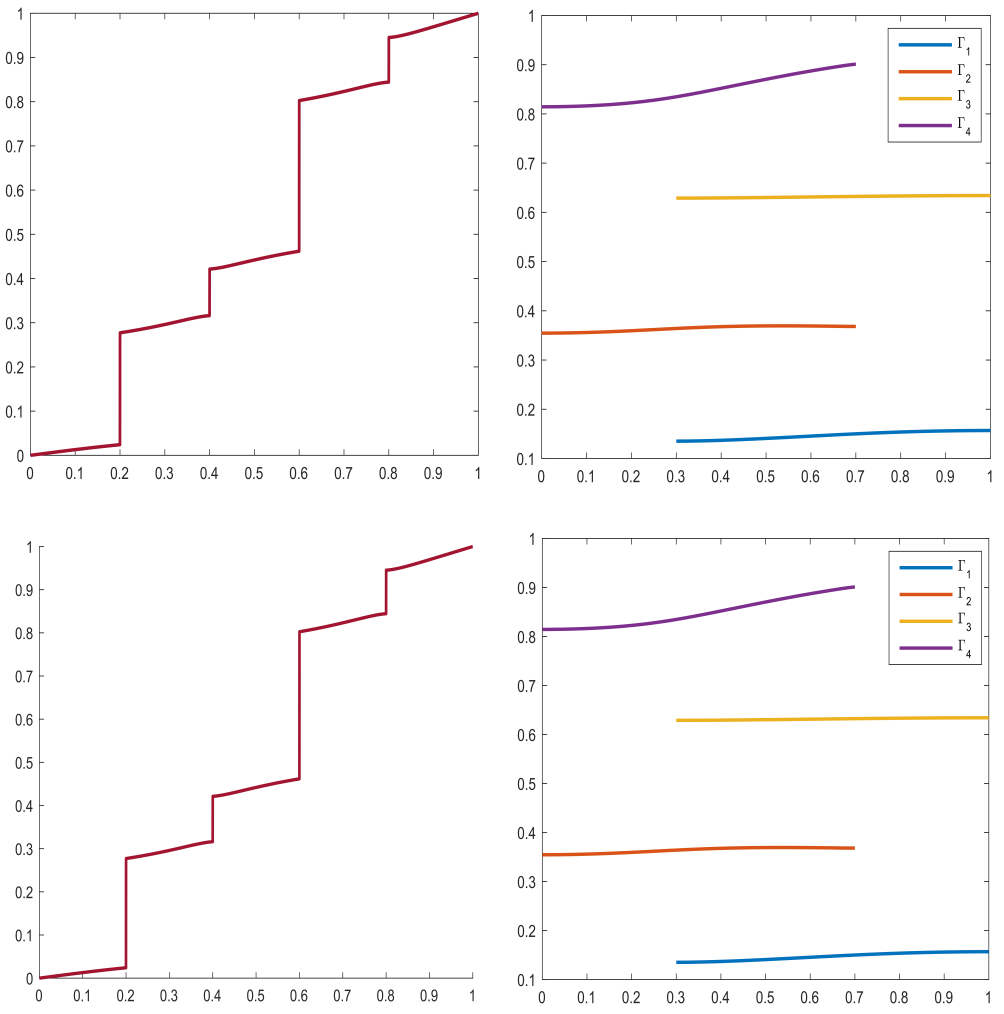

Fig. 21 Example 2 (Section 5.4.2). Immersed fractures; configuration A, primal-primal approximation (top) and mixed-primal approximation (bottom).

and B), by varying the value of the permeability $v_{\Gamma}^{\tau}$ on the partially permeable fractures $\Gamma_{1}$ and $\Gamma_{3}$ and the boundary conditions as illustrated in Figure 20. At the extremities of the fractures that are non-immersed, i.e., $\partial \Gamma \cap \partial \Omega$, we impose boundary conditions that are consistent with those imposed on $\partial \Omega$ at that point. In both cases we consider an isotropic bulk permeability tensor, i.e., $v=\mathbf{I}$ and we assume that all the fractures have aperture $\ell_{\Gamma}=0.01$. Moreover, we take the forcing terms $f=f_{\Gamma}=0$, so that the flow is only generated by the boundary conditions. Finally, we choose the parameter $\xi=0.55$. Our results have been obtained with Cartesian grids aligned with the fractures, consisting of 26243 elements; this is approximately the same as in [4]. We remark that each immersed fracture tips coincides with a mesh vertex (in the case when the fracture ends at an edge of an element, the tip is considered as an additional vertex 
Bulk pressure field and streamlines
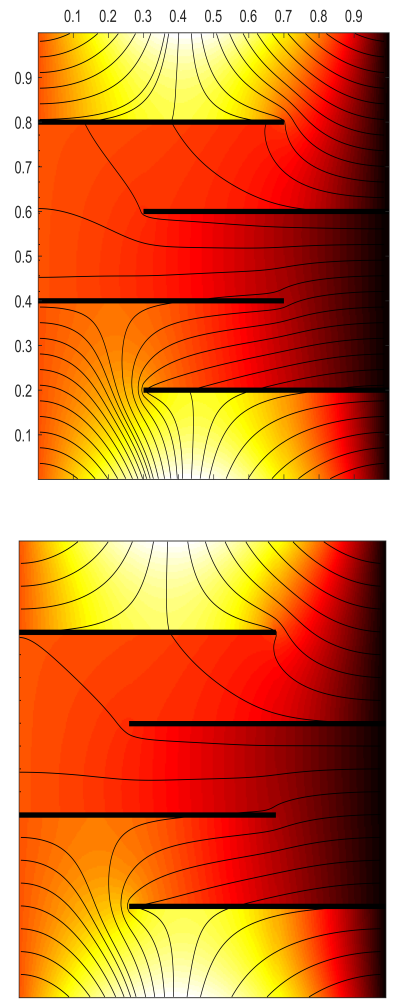

Bulk pressure along $x=0.65$
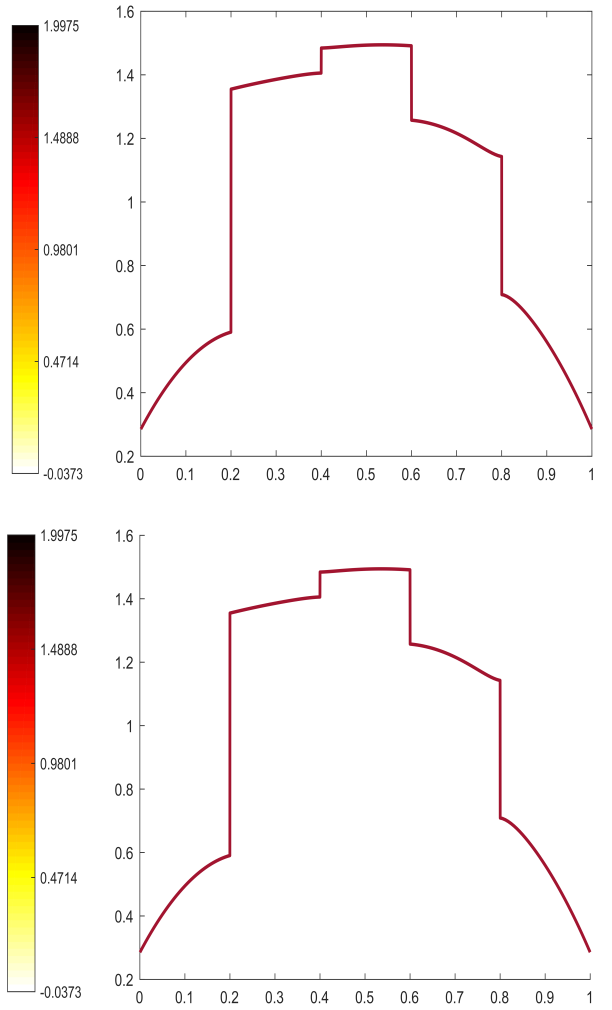

Pressure in the fractures
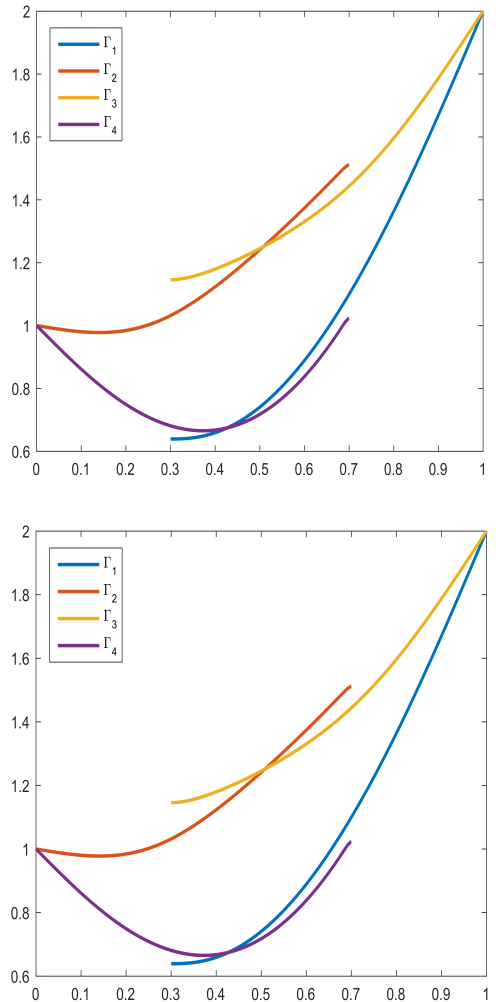

Fig. 22 Example 2 (Section 5.4.2). Immersed fractures; configuration B, primal-primal approximation (top) and mixed-primal approximation (bottom).

for the quadrilateral, which then becomes a pentagon). For both the (PP) and (MP) approximations we choose uniform linear polynomial degrees for both the bulk and fracture problems. In Figure 21 we show the results obtained with the (PP) and (MP) methods for configuration A; in Figure 22 we show analogous results for the configuration B. In particular, in both figures, we report the pressure field in the bulk with the streamlines of the velocity (left), the value of the bulk pressure along the line $x=0.65$ (middle) and the pressure field inside the four fractures (right). The top line of each figure encloses the results obtained with the (PP) approximation, while the bottom line presents those obtained with the (MP) method. For both the (PP) and (MP) schemes, our results are in perfect agreement with those obtained in [4], thus showing that our approximation schemes can be easily extended to the treatment of more complex situations. Moreover, for this example, we observe that the (PP) and (MP) methods deliver the same level of accuracy.

\subsubsection{Example 3: Network of intersecting fractures}

We conclude with a test case, already presented in [14], that aims at investigating the capability of our method for dealing with a network of intersecting fractures, which is also totally immersed in the bulk domain. In order to proceed, we need to complement our mathematical model (29) with some conditions at the intersection points, prescribing the behaviour of the fluid. In particular, we impose pressure continuity and flux conservation, as in [96, 49, 56]. At the immersed tips we impose the no flux condition $v_{\Gamma}^{\tau} \nabla_{\tau} p_{\Gamma} \cdot \tau=0$ as above. We also mention that this numerical experiment was first presented in [18] employing the mimetic finite difference method. Here, we employ a suitable modification of the primal-primal scheme, which is able to handle intersecting fractures by virtue of an appropriate definition of jump and average operators at the intersection points. We refer to [15] for a detailed analysis of this scheme.

In the numerical simulations, we consider the bulk domain $\Omega=(0,1)^{2}$ and the network made of 10 intersecting fractures that is shown in Figure 23(a). We impose homogeneous Dirichlet boundary conditions on the whole $\partial \Omega$ and 


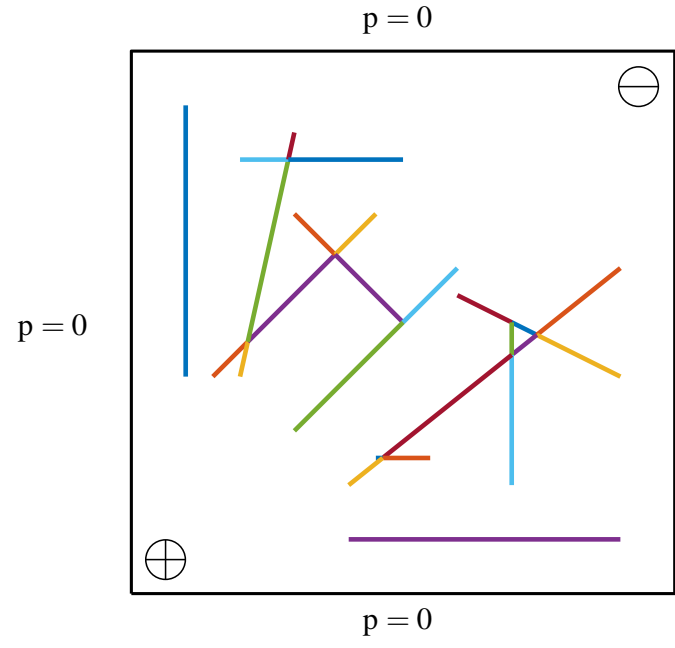

(a) Computational domain

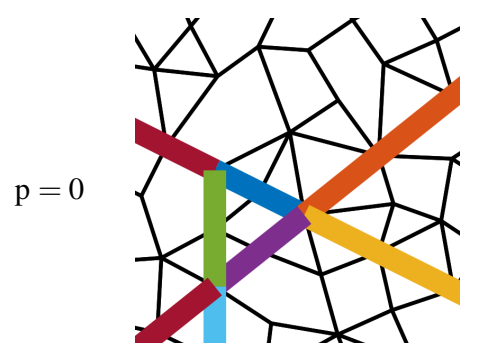

(b) Mesh detail

define the source terms in the bulk and in the fracture as

$$
f(x, y)=\left\{\begin{array}{ll}
10 & \text { if }(x-0.1)^{2}+(y-0.1)^{2} \leq 0.04, \\
-10 & \text { if }(x-0.9)^{2}+(y-0.9)^{2} \leq 0.04,
\end{array} \quad f_{\Gamma}=0,\right.
$$

respectively. We note that the source term in the bulk is defined so that a source is present in the lower left corner of the domain and a sink in its top right corner. We assume that the porous medium in the bulk is isotropic and homogeneous, i.e., $v=$ Id. With the aim of testing the behaviour of the bulk pressure depending on the permeability properties of the fracture network, we consider three different configurations:

1. No fractures are present in the porous medium;

2. Permeable network: all the fractures have high permeability properties with $v_{\Gamma}^{\tau}=v_{\Gamma}^{n}=1000$ and constant thickness $\ell_{\Gamma}=0.01$;

3. Impermeable network: all the fractures have blocking properties with $v_{\Gamma}^{\tau}=v_{\Gamma}^{n}=0.001$ and constant thickness $\ell_{\Gamma}=0.01$.

In Figure 23(b) we show a detail of the polygonal mesh conforming to the fracture network that we employed for the simulations. The discrete pressures for the problem in the bulk, obtained with the primal-primal approximation, in the three cases outlined above, are presented in Figure 24. In particular, one may observe that, when the network is permeable, the bulk pressure is only marginally affected by the presence of the fractures, so that it reaches maximum

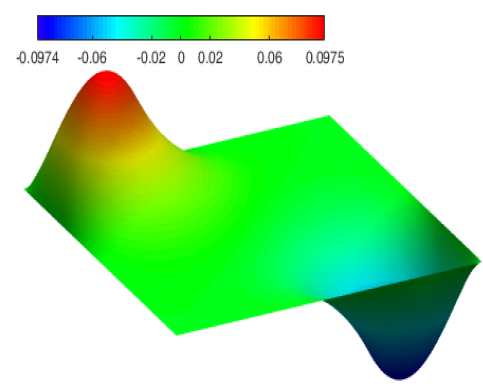

(a) No fractures

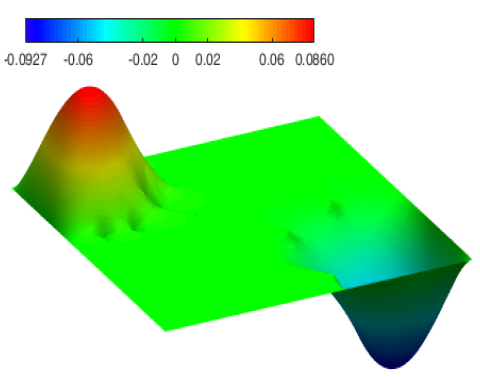

(b) Permeable

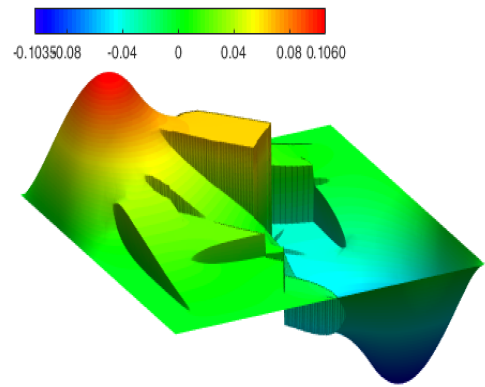

(c) Impermeable

Fig. 24 Example 3 (Section 5.4.3). Network of intersecting fractures: discrete pressure in the bulk for the three test cases, no fractures (left), permeable network $v_{\Gamma}^{\tau}=v_{\Gamma}^{n}=1000$ (middle), impermeable network $v_{\Gamma}^{\tau}=v_{\Gamma}^{n}=0.001$ (right). Taken from [14]. 
and minimum values that are only slightly lower than those of the non-fractured case (see the comparison between Figure 24(a) and Figure 24(b)). In contrast, in the impermeable case, jumps of the bulk pressure across the fractures are clearly observed, cf., Figure 24(c). Finally, we note that our results are in good agreement with those obtained in [96].

\section{Conclusions}

In this work we have provided a comprehensive review of the current development of PolyDG methods for geophysical applications, addressing as paradigmatic applications the numerical modeling of seismic wave propagation and fracture reservoir simulations. After having recalled the theoretical background of the analysis of PolyDG methods (cf. Section 2), in Section 3 we discussed the issue of efficiently implementing DG methods on polytopic meshes, addressing in detail the issue of numerical quadrature and recalling the main results contained in [22], where a new quadrature free algorithm for the numerical evaluation of the integrals required to assemble the mass and stiffness matrices has been proposed. More precisely, a cubature method, which does not require the definition of a set of nodes and weights on the domain of integration, and allows for the exact integration of polynomial functions based on evaluating the integrand only at the vertices of the polytopic integration domain, is presented and tested in both two- and three-dimensions. This approach shows a remarkable gain in terms of CPU time with respect to classical quadrature rules, while maintaining the same degree of accuracy. In Section 4 we presented PolyDG methods for the approximate solution of the elastodynamics equations on computational meshes consisting of polytopic elements. We analysed the well-posedness of the numerical formulation and proved $h p$-version a priori error estimates for the semidiscrete scheme. The fully discrete method is then obtained based on employing the leap-frog scheme for the time discretization. To test the numerical performance and fully exploit the flexibility in the process of mesh design offered by polytopic elements numerical experiments have been presented. Section 5 focused on the problem of modeling the flow in a fractured porous medium. For ease of presentation and analysis we have assumed the medium to be cut by a single non-immersed fracture and have reviewed the unified development and analysis of PolyDG methods for this class of problems. These error bounds have been validated through numerical tests. Moreover, we have demonstrated that our approach can be extended to handle networks of partially immersed fractures and networks of intersecting fractures, cf. [15]. To conclude we mention that the current developments of PolyDG methods, not discussed here for the sake of brevity, include the exploitation of agglomeration-based algorithms to design multilevel and multigrid methods for the efficient iterative solution of the (linear) system of equations stemming from the PolyDG discretization. Indeed, multigrid/multilevel solvers require the definition of a succession of coarse grids, based on the original 'fine' grid. The process of defining the coarser grids involves what is called agglomeration, i.e., the combination of several nodes or control volumes or coefficients from the original grid. In this context, the flexibility offered by polytopic grids can be fully exploited. Some pioneering works on the analysis of agglomeration-based multigrid/multilevel solvers and preconditioners can be found in [21, 30, 23]; cf. also the classical approach based on a sequence of simplicial/quadrilateral meshes [31].

\section{References}

1. J. Aghili, D. A. Di Pietro, and B. Ruffini. An $h p$-hybrid high-order method for variable diffusion on general meshes. Comput. Methods Appl. Math., 17(3):359-376, 2017.

2. C. Alboin, J. Jaffré, J. E. Roberts, and C. Serres. Modeling fractures as interfaces for flow and transport in porous media. In Fluid flow and transport in porous media: mathematical and numerical treatment (South Hadley, MA, 2001), volume 295 of Contemp. Math., pages 13-24. Amer. Math. Soc., Providence, RI, 2002.

3. C. Alboin, J. Jaffré, J. E. Roberts, X. Wang, and C. Serres. Domain decomposition for some transmission problems in flow in porous media. In Numerical treatment of multiphase flows in porous media, volume 552 of Lecture Notes in Phys., pages 22-34. Springer, Berlin, 2000.

4. P. Angot, F. Boyer, and F. Hubert. Asymptotic and numerical modelling of flows in fractured porous media. M2AN Math. Model. Numer. Anal., 43(2):239-275, 2009.

5. P. Antonietti, M. Verani, C. Vergara, and S. Zonca. Numerical solution of fluid-structure interaction problems by means of a high order Discontinuous Galerkin method on polygonal grids. Finite Elem. Anal. Des., 159:1-14, 2019.

6. P. F. Antonietti, B. Ayuso de Dios, I. Mazzieri, and A. Quarteroni. Stability analysis of discontinuous Galerkin approximations to the elastodynamics problem. J. Sci. Comput., 68:143-170, 2016. 
7. P. F. Antonietti, L. Beirão da Veiga, N. Bigoni, and M. Verani. Mimetic finite differences for nonlinear and control problems. Math. Models Methods Appl. Sci., 24(8):1457-1493, 2014.

8. P. F. Antonietti, L. Beirão da Veiga, D. Mora, and M. Verani. A stream Virtual Element formulation of the Stokes problem on polygonal meshes. SIAM J. Numer. Anal., 52(1):386-404, 2014.

9. P. F. Antonietti, L. Beirão da Veiga, S. Scacchi, and M. Verani. A C1 Virtual Element Method for the Cahn-Hilliard equation with polygonal meshes. SIAM J. Numer. Anal., 54(1):34-56, 2016.

10. P. F. Antonietti, F. Bonaldi, and I. Mazzieri. Simulation of $3 \mathrm{~d}$ elasto-acoustic wave propagation based on a discontinuous galerkin spectral element method. Internat. J. Numer. Methods Engrg., 2019.

11. P. F. Antonietti, F. Bonaldi, and I. Mazzieri. A high-order discontinuous Galerkin approach to the elasto-acoustic problem. Comput. Methods Appl. Mech. Engrg., 358:112634, 29, 2020.

12. P. F. Antonietti, F. Brezzi, and L. D. Marini. Bubble stabilization of discontinuous Galerkin methods. Comput. Methods Appl. Mech. Engrg., 198(21-26):1651-1659, 2009.

13. P. F. Antonietti, A. Cangiani, J. Collis, Z. Dong, E. H. Georgoulis, S. Giani, and P. Houston. Review of Discontinuous Galerkin Finite Element Methods for Partial Differential Equations on Complicated Domains. Lect. Notes Comput. Sci. Eng., 114:281 - $310,2015$.

14. P. F. Antonietti, C. Facciolà, A. Russo, and M. Verani. Discontinuous Galerkin Approximation of Flows in Fractured Porous Media on Polytopic Grids. SIAM J. Sci. Comput., 41(1):A109-A138, 2019.

15. P. F. Antonietti, C. Facciolà, and M. Verani. Polytopic discontinuous Galerkin approximation of flows in porous media with networks of fractures. MOX Report 8/2020. Submitted., 2020.

16. P. F. Antonietti, C. Facciolà, and M. Verani. Unified formulation for polytopic discontinuous galerkin approximation of flows in fractured porous media. Mathematics in Engineering, 2(1):340-385, 2020.

17. P. F. Antonietti, A. Ferroni, I. Mazzieri, R. Paolucci, A. Quarteroni, C. Smerzini, and M. Stupazzini. Numerical modeling of seismic waves by discontinuous spectral element methods. In 43-ème Congrès National d'Analyse Numérique, CANUM2016, volume 61 of ESAIM Proc. Surveys, pages 1-37. EDP Sci., Les Ulis, 2018.

18. P. F. Antonietti, L. Formaggia, A. Scotti, M. Verani, and N. Verzotti. Mimetic finite difference approximation of flows in fractured porous media. M2AN Math. Model. Numer. Anal., 50(3):809-832, 2016.

19. P. F. Antonietti, S. Giani, and P. Houston. $h p$-version Composite Discontinuous Galerkin methods for elliptic problems on complicated domains. SIAM J. Sci. Comput., 35(3):A1417-A1439, 2013.

20. P. F. Antonietti, S. Giani, and P. Houston. Domain decomposition preconditioners for discontinuous Galerkin methods for elliptic problems on complicated domains. J. Sci. Comput., 60(1):203-227, 2014.

21. P. F. Antonietti, P. Houston, X. Hu, M. Sarti, and M. Verani. Multigrid algorithms for hp-version interior penalty discontinuous Galerkin methods on polygonal and polyhedral meshes. Calcolo, 54(4):1169-1198, 2017.

22. P. F. Antonietti, P. Houston, and G. Pennesi. Fast numerical integration on polytopic meshes with applications to discontinuous Galerkin finite element methods. J. Sci. Comput., 77:1339-1370, 2018.

23. P. F. Antonietti, P. Houston, G. Pennesi, and E. Süli. An agglomeration-based massively parallel non-overlapping additive Schwarz preconditioner for high-order discontinuous Galerkin methods on polytopic grids. Math. Comp., 2020. https://doi.org/10.1090/mcom/3510. In press.

24. P. F. Antonietti, G. Manzini, and M. Verani. The fully nonconforming Virtual Element Method for biharmonic problems. Math. Models Methods Appl. Sci., 28(02):387-407, 2018. M3AS Math. Models Methods Appl. Sci.

25. P. F. Antonietti, C. Marcati, I. Mazzieri, and A. Quarteroni. High order discontinuous Galerkin methods on simplicial elements for the elastodynamics equation. Numer. Algorithms, 71(1):181-206, 2016.

26. P. F. Antonietti and I. Mazzieri. High-order Discontinuous Galerkin methods for the elastodynamics equation on polygonal and polyhedral meshes. Comput. Methods Appl. Mech. Engrg., 342:414-437, 2018.

27. P. F. Antonietti, I. Mazzieri, N. Dal Santo, and A. Quarteroni. A high-order discontinuous Galerkin approximation to ordinary differential equations with applications to elastodynamics. IMA J. Numer. Anal., 38(4):1709-1734, 2018.

28. P. F. Antonietti, I. Mazzieri, M. Muhr, V. Nikolić, and B. Wohlmuth. A high-order discontinuous Galerkin method for nonlinear sound waves. Submitted, 2019.

29. P. F. Antonietti, I. Mazzieri, A. Quarteroni, and F. Rapetti. Non-conforming high order approximations of the elastodynamics equation. Comput. Methods Appl. Mech. Engrg., 209:212-238, 2012.

30. P. F. Antonietti and G. Pennesi. V-cycle multigrid algorithms for discontinuous Galerkin methods on non-nested polytopic meshes. J. Sci. Comput., 78(1):625-652, 2019.

31. P. F. Antonietti, M. Sarti, and M. Verani. Multigrid algorithms for $h p$-discontinuous Galerkin discretizations of elliptic problems. SIAM J. Numer. Anal., 53(1):598-618, 2015.

32. D. N. Arnold. An interior penalty finite element method with discontinuous elements. SIAM J. Numer. Anal., 19(4):742-760, 1982.

33. D. N. Arnold, F. Brezzi, B. Cockburn, and L. D. Marini. Unified analysis of discontinuous Galerkin methods for elliptic problems. SIAM J. Numer. Anal., 39(5):1749-1779, 2001/02.

34. D. N. Arnold, F. Brezzi, R. S. Falk, and L. D. Marini. Locking-free Reissner-Mindlin elements without reduced integration. Comput. Methods Appl. Mech. Engrg., 196(37-40):3660-3671, 2007.

35. B. Ayuso de Dios, K. Lipnikov, and G. Manzini. The nonconforming Virtual Element Method. ESAIM Math. Model. Numer. Anal., 50(3):879-904, 2016.

36. F. Bassi, L. Botti, and A. Colombo. Agglomeration-based physical frame dG discretizations: an attempt to be mesh free. Math. Models Methods Appl. Sci., 24(8):1495-1539, 2014.

37. F. Bassi, L. Botti, A. Colombo, D. A. Di Pietro, and P. Tesini. On the flexibility of agglomeration based physical space discontinuous Galerkin discretizations. J. Comput. Phys., 231(1):45-65, 2012.

38. F. Bassi, L. Botti, A. Colombo, and S. Rebay. Agglomeration based discontinuous Galerkin discretization of the Euler and NavierStokes equations. Comput. \& Fluids, 61:77-85, 2012. 
39. L. Beirão da Veiga, F. Brezzi, A. Cangiani, G. Manzini, L. D. Marini, and A. Russo. Basic principles of Virtual Element Methods. Math. Models Methods Appl. Sci., 23(1):199 - 214, 2013.

40. L. Beirão da Veiga, F. Brezzi, F. Dassi, L. D. Marini, and A. Russo. A family of three-dimensional virtual elements with applications to magnetostatics. SIAM J. Numer. Anal., 56(5):2940-2962, 2018.

41. L. Beirão da Veiga, F. Dassi, and A. Russo. High-order virtual element method on polyhedral meshes. Comput. Math. Appl., 74(5):1110-1122, 2017.

42. L. Beirão da Veiga, K. Lipnikov, and G. Manzini. The Mimetic Finite Difference method for elliptic problems, volume 11. Springer, Cham, 2014.

43. L. Beirão da Veiga, D. Mora, and G. Rivera. Virtual elements for a shear-deflection formulation of Reissner-Mindlin plates. Math. Comp., 88(315):149-178, 2019.

44. L. Beirão da Veiga, D. Mora, and G. Vacca. The Stokes complex for virtual elements with application to Navier-Stokes flows. J. Sci. Comput., 81(2):990-1018, 2019.

45. L. Beirão da Veiga, A. Russo, and G. Vacca. The virtual element method with curved edges. ESAIM Math. Model. Numer. Anal., 53(2):375-404, 2019.

46. L. Beirão da Veiga, F. Brezzi, L. Marini, and A. Russo. Virtual Element Method for general second-order elliptic problems on polygonal meshes. Math. Models Methods Appl. Sci., 26(04):729-750, 2016.

47. M. F. Benedetto, S. Berrone, A. Borio, S. Pieraccini, and S. Scialò. A hybrid mortar virtual element method for discrete fracture network simulations. J. Comput. Phys., 306:148-166, 2016.

48. M. F. Benedetto, S. Berrone, S. Pieraccini, and S. Scialò. The virtual element method for discrete fracture network simulations. Comput. Methods Appl. Mech. Engrg., 280:135-156, 2014.

49. M. F. Benedetto, S. Berrone, and S. Scialò. A globally conforming method for solving flow in discrete fracture networks using the virtual element method. Finite Elem. Anal. Des., 109:23-36, 2016.

50. A. Bermúdez, P. Gamallo, L. Hervella-Nieto, and R. Rodríguez. Finite element analysis of pressure formulation of the elastoacoustic problem. Numer. Math., 95:29-51, 2003.

51. A. Bermúdez, L. Hervella-Nieto, and R. Rodríguez. Finite element computation of three-dimensional elastoacoustic vibrations. $J$ SOUND VIB, 219:279-306, 1999.

52. J. Bielak, O. Ghattas, and E. Kim. Parallel octree-based finite element method for large-scale earthquake ground motion simulation. CMES - Computer Modeling in Engineering and Sciences, 10:99-112, 2005.

53. L. Botti and D. A. Di Pietro. Assessment of hybrid high-order methods on curved meshes and comparison with discontinuous Galerkin methods. J. Comput. Phys., 370:58-84, 2018.

54. L. Botti, D. A. Di Pietro, and J. Droniou. A hybrid high-order method for the incompressible Navier-Stokes equations based on Temam's device. J. Comput. Phys., 376:786-816, 2019.

55. M. Botti, D. A. Di Pietro, and P. Sochala. A hybrid high-order method for nonlinear elasticity. SIAM J. Numer. Anal., 55(6):2687$2717,2017$.

56. K. Brenner, J. Hennicker, R. Masson, and P. Samier. Gradient discretization of hybrid-dimensional Darcy flow in fractured porous media with discontinuous pressures at matrix-fracture interfaces. IMA J. Numer. Anal., 37(3):1551-1585, 2016.

57. F. Brezzi, T. J. Hughes, L. D. Marini, and A. Masud. Mixed discontinuous Galerkin methods for darcy flow. J. Sci. Comput., 22(1-3):119-145, 2005.

58. F. Brezzi, K. Lipnikov, and M. Shashkov. Convergence of the Mimetic Finite Difference method for diffusion problems on polyhedral meshes. SIAM J. Numer. Anal., 43(5):1872-1896 (electronic), 2005.

59. F. Brezzi, K. Lipnikov, and M. Shashkov. Convergence of Mimetic Finite Difference method for diffusion problems on polyhedral meshes with curved faces. Math. Models Methods Appl. Sci., 16(2):275-297, 2006.

60. F. Brezzi, K. Lipnikov, and V. Simoncini. A family of Mimetic Finite Difference methods on polygonal and polyhedral meshes. Math. Models Methods Appl. Sci., 15(10):1533-1551, 2005.

61. E. Burman, P. Hansbo, M. G. Larson, and K. Larsson. Cut finite elements for convection in fractured domains. Computers \& Fluids, 179:726-734, 2019.

62. A. Cangiani, Z. Dong, and E. Georgoulis. $h p$-Version discontinuous Galerkin methods on essentially arbitrarily-shaped elements. Submitted for publication, 2019.

63. A. Cangiani, Z. Dong, and E. H. Georgoulis. $h p$-version space-time discontinuous Galerkin methods for parabolic problems on prismatic meshes. SIAM J. Sci. Comput., 39(4):A1251-A1279, 2017.

64. A. Cangiani, Z. Dong, E. H. Georgoulis, and P. Houston. $h p$-version discontinuous Galerkin methods for advection-diffusion-reaction problems on polytopic meshes. ESAIM Math. Model. Numer. Anal., 50(3):699-725, 2016.

65. A. Cangiani, Z. Dong, E. H. Georgoulis, and P. Houston. hp-Version Discontinuous Galerkin Methods on Polygonal and Polyhedral Meshes. SpringerBriefs in Mathematics, 2017.

66. A. Cangiani, Z. Dong, E. H. Georgoulis, and P. Houston. hp-version discontinuous Galerkin methods on polytopic meshes. SpringerBriefs in Mathematics. Springer International Publishing, 2017.

67. A. Cangiani, E. H. Georgoulis, and P. Houston. $h p$-version discontinuous Galerkin methods on polygonal and polyhedral meshes. Math. Models Methods Appl. Sci., 24(10):2009-2041, 2014.

68. A. Cangiani, G. Manzini, and O. J. Sutton. Conforming and nonconforming Virtual Element Methods for elliptic problems. IMA J. Numer. Anal., 37(3):1317-1354, 2017.

69. P. Castillo, B. Cockburn, I. Perugia, and D. Schötzau. An a priori error analysis of the local discontinuous Galerkin method for elliptic problems. SIAM J. Numer. Anal., 38(5):1676-1706, 2000.

70. E. Chaljub, Y. Capdeville, and J. P. Vilotte. Solving elastodynamics in a fluid-solid heterogeneous sphere: a parallel spectral element approximation on non-conforming grids. J. Comput. Phys., 187:457-491, 2003.

71. F. Chave, D. A. Di Pietro, and L. Formaggia. A hybrid high-order method for Darcy flows in fractured porous media. SIAM J. Sci. Comput., 40(2):A1063-A1094, 2018. 
72. F. Chave, D. A. Di Pietro, F. Marche, and F. Pigeonneau. A hybrid high-order method for the Cahn-Hilliard problem in mixed form. SIAM J. Numer. Anal., 54(3):1873-1898, 2016.

73. F. A. Chave, D. Di Pietro, and L. Formaggia. A Hybrid High-Order method for Darcy flows in fractured porous media. SIAM J. Sci. Comput., 40(2):A1063-A1094, 2018.

74. E. B. Chin, J. B. Lasserre, and N. Sukumar. Numerical integration of homogeneous functions on convex and nonconvex polygons and polyhedra. Comput. Mech., 56(6):967 - 981, 2015.

75. B. Cockburn, B. Dond, and J. Guzmán. A superconvergent LDG-hybridizable Galerkin method for second-order elliptic problems. Math. Comp., 77(264):1887-1916, 2008.

76. B. Cockburn, J. Gopalakrishnan, and R. Lazarov. Unified hybridization of discontinuous Galerkin, mixed, and continuous Galerkin methods for second order elliptic problems. SIAM J. Numer. Anal., 47(2):1319-1365, 2009.

77. B. Cockburn, J. Gopalakrishnan, and F.-J. Sayas. A projection-based error analysis of HDG methods. Math. Comp., 79(271):1351$1367,2010$.

78. B. Cockburn, J. Guzmán, and H. Wang. Superconvergent discontinuous Galerkin methods for second-order elliptic problems. Math. Comp., 78(265):1-24, 2009.

79. B. Cockburn and C.-W. Shu. The local discontinuous Galerkin method for time-dependent convection-diffusion systems. SIAM J. Numer. Anal., 35(6):2440-2463, 1998.

80. G. Cohen, X. Ferrieres, and S. Pernet. A spatial high-order hexahedral discontinuous galerkin method to solve maxwell's equations in time domain. J. Comput. Phys., 217(2):340 - 363, 2006.

81. C. D'Angelo and A. Scotti. A mixed finite element method for darcy flow in fractured porous media with non-matching grids. ESAIM Math. Model. Numer. Anal., 46(02):465-489, 2012.

82. J. D. De Basabe and M. K. Sen. A comparison of finite-difference and spectral-element methods for elastic wave propagation in media with a fluid-solid interface. Geophys. J. Int., 200:278-298, 2015.

83. J. D. De Basabe, M. K. Sen, and M. F. Wheeler. The interior penalty discontinuous Galerkin method for elastic wave propagation: grid dispersion. Geophys. J. Int., 175(1):83-93, 2008.

84. D. A. Di Pietro and J. Droniou. A hybrid high-order method for Leray-Lions elliptic equations on general meshes. Math. Comp., 86(307):2159-2191, 2017.

85. D. A. Di Pietro and J. Droniou. $W^{s, p}$-approximation properties of elliptic projectors on polynomial spaces, with application to the error analysis of a hybrid high-order discretisation of Leray-Lions problems. Math. Models Methods Appl. Sci., 27(5):879-908, 2017.

86. D. A. Di Pietro and A. Ern. Hybrid high-order methods for variable-diffusion problems on general meshes. C. R. Math. Acad. Sci. Paris, 353(1):31-34, 2015.

87. D. A. Di Pietro and S. Krell. A hybrid high-order method for the steady incompressible Navier-Stokes problem. J. Sci. Comput., 74(3):1677-1705, 2018.

88. J. Dolbow, N. Moes, and T. Belytschko. An extended finite element method for modeling crack growth with frictional contact. Comput. Methods Appl. Mech. Engrg., 190(51-52):6825-6846, 2001.

89. V. Dolean, H. Fol, S. Lanteri, and R. Perrussel. Solution of the time-harmonic maxwell equations using discontinuous galerkin methods. J. Comput. Appl. Math., 218(2):435 - 445, 2008.

90. J. Droniou, R. Eymard, and R. Herbin. Gradient schemes: generic tools for the numerical analysis of diffusion equations. ESAIM Math. Model. Numer. Anal., 50(3):749-781, 2016.

91. M. Dumbser and M. Käser. An arbitrary high-order discontinuous Galerkin method for elastic waves on unstructured meshes - II. The three-dimensional isotropic case. Geophys. J. Int., 167(1):319-336, 2006.

92. A. Ferroni, P. Antonietti, I. Mazzieri, and A. Quarteroni. Dispersion-dissipation analysis of 3-d continuous and discontinuous spectral element methods for the elastodynamics equation. Geophys. J. Int., 211(3):1554-1574, 2017.

93. A. Fichtner, H. Igel, H.-P. Bunge, and B. L. N. Kennett. Simulation and inversion of seismic wave propagation on continental scales based on a spectral-element method. JNAIAM J. Numer. Anal. Ind. Appl. Math., 4(1-2):11-22, jun 2009.

94. B. Flemisch, A. Fumagalli, and A. Scotti. A review of the XFEM-based approximation of flow in fractured porous media. In Advances in Discretization Methods, pages 47-76. Springer, 2016.

95. L. Formaggia, A. Fumagalli, A. Scotti, and P. Ruffo. A reduced model for darcy's problem in networks of fractures. ESAIM: Mathematical Modelling and Numerical Analysis, 48(4):1089-1116, 2014.

96. L. Formaggia, A. Scotti, and F. Sottocasa. Analysis of a mimetic finite difference approximation of flows in fractured porous media. ESAIM Math. Model. Numer. Anal., 52(2):595-630, 2018.

97. T.-P. Fries and T. Belytschko. The extended/generalized finite element method: an overview of the method and its applications. Internat. J. Numer. Methods Engrg., 84(3):253-304, 2010.

98. N. Frih, J. E. Roberts, and A. Saada. Modeling fractures as interfaces: a model for Forchheimer fractures. Comput. Geosci., 12(1):91$104,2008$.

99. A. Fumagalli and A. Scotti. A numerical method for two-phase flow in fractured porous media with non-matching grids. Advances in Water Resources, 62, Part C:454-464, 2013.

100. P. Galvez, J.-P. Ampuero, L. A. Dalguer, S. N. Somala, and T. Nissen-Meyer. Dynamic earthquake rupture modelled with an unstructured 3-D spectral element method applied to the 2011 M9 Tohoku earthquake. Geophys. J. Int., 198(2):1222-1240, 2014.

101. A. Gerstenberger and A. W. Wall. An extended finite element method/Lagrange multiplier based approach for fluid-structure interaction. Comput. Methods Appl. Mech. Engrg., 197(19 - 20):1699 - 1714, 2008.

102. F. X. Giraldo and T. Warburton. A high-order triangular discontinuous galerkin oceanic shallow water model. Internat. J. Numer. Methods Fluids, (7):899-925, 2008.

103. W. Hackbusch and S. A. Sauter. Composite Finite Elements for problems containing small geometric details. Part II: Implementation and numerical results. Comput. Visual Sci., 1(4):15-25, 1997.

104. W. Hackbusch and S. A. Sauter. Composite Finite Elements for the approximation of PDEs on domains with complicated microstructures. Numer. Math., 75(4):447-472, 1997. 
105. D. J. Holdych, D. R. Noble, and R. B. Secor. Quadrature rules for triangular and tetrahedral elements with generalized functions. Internat. J. Numer. Methods Engrg., 73(9):1310 - 1327, 2015.

106. J. Hyman, M. Shashkov, and S. Steinberg. The numerical solution of diffusion problems in strongly heterogeneous non-isotropic materials. J. Comput. Phys., 132(1):130-148, 1997.

107. J. Jaffré, M. Mnejja, and J. Roberts. A discrete fracture model for two-phase flow with matrix-fracture interaction. Procedia Computer Science, 4:967 -973, 2011.

108. G. Karypis and V. Kumar. A fast and high quality multilevel scheme for partitioning irregular graphs. SIAM J. Sci. Comput., 20(1):359 $-392,1998$.

109. G. Karypis and V. Kumar. Metis: Unstructured graph partitioning and sparse matrix ordering system, version 4.0. http: //www . c. . umn . edu/ metis, 2009.

110. M. Käser and M. Dumbser. An arbitrary high-order discontinuous Galerkin method for elastic waves on unstructured meshes - I: The two-dimensional isotropic case with external source terms. Geophys. J. Int., 166(2):855-877, 2006.

111. M. Käser and M. Dumbser. A highly accurate discontinuous Galerkin method for complex interfaces between solids and moving fluids. Geophysics, 73:T23-T35, 2008.

112. D. Komatitsch and J. Tromp. Spectral-element simulations of global seismic wave propagation-I. Validation. Geophys. J. Int., 149(2):390-412, 2002.

113. D. Komatitsch and J. Tromp. Spectral-element simulations of global seismic wave propagation-II. Three-dimensional models, oceans, rotation and self-gravitation. Geophys. J. Int., 150(1):303-318, 2002.

114. J. B. Lasserre. Integration on a convex polytope. Proc. Amer. Math. Soc., 126(8):2433 - 2441, 1998.

115. C.-J. Li, P. Lambertu, and C. Dagnino. Numerical integration over polygons using an eight-node quadrilateral spline finite element. J. Comput. Appl. Math., 233(2):279 - 292, 2009.

116. J. N. Lyness and G. Monegato. Quadrature rules for regions having regular hexagonal symmetry. SIAM J. Numer. Anal., 14(2):283 295, 1977.

117. J. Ma, V. Rokhlin, and S. Wandzura. Generalized Gaussian quadrature of systems of arbitrary functions. SIAM J. Numer. Anal., 33(3):971 - 996, 1996.

118. V. Martin, J. Jaffré, and J. E. Roberts. Modeling fractures and barriers as interfaces for flow in porous media. SIAM J. Sci. Comput., 26(5):1667-1691, 2005.

119. A. Masud and T. J. Hughes. A stabilized mixed finite element method for darcy flow. Comput. Methods Appl. Mech. Engrg., 191(39-40):4341-4370, 2002.

120. I. Mazzieri, M. Stupazzini, R. Guidotti, and C. Smerzini. SPEED: Spectral Elements in Elastodynamics with Discontinuous Galerkin: a non-conforming approach for 3D multi-scale problems. Internat. J. Numer. Methods Engrg., 95(12):991-1010, 2013.

121. E. D. Mercerat and N. Glinsky. A nodal high-order discontinuous Galerkin method for elastic wave propagation in arbitrary heterogeneous media. Geophys. J. Int., 201(2):1101-1118, 2015.

122. N. Moes, J. Dolbow, and T. Belytschko. A finite element method for crack growth without remeshing. Int. J. Numer. Meth. Eng., 46(1):131 - 150, 1999.

123. S. E. Mousavi and N. Sukumar. Numerical integration of polynomials and discontinuous functions on irregular convex polygons and polyhedrons. Comput. Mech., 47(5):535 - 554, 2011.

124. S. E. Mousavi, H. Xiao, and N. Sukumar. Generalized Gaussian quadrature rules on arbitrary polygons. Internat. J. Numer. Methods Engrg., 82(1):99 - 113, 2010.

125. W. A. Mulder, E. Zhebel, and S. Minisini. Time-stepping stability of continuous and discontinuous finite-element methods for 3-d wave propagation. Geophys. J. Int., 196(2):1123-1133, 2014.

126. S. Natarajan, S. Bordas, and D. R. Mahapatra. Numerical integration over arbitrary polygonal domains based on Schwarz-Christoffel conformal mapping. Internat. J. Numer. Methods Engrg., 80(1):103 - 134, 2009.

127. I. Perugia and D. Schötzau. An $h p$-analysis of the local discontinuous galerkin method for diffusion problems. J. Sci. Comput., 17(1):561-571, 2002.

128. I. Perugia and D. Schötzau. The $h p$-local discontinuous Galerkin method for low-frequency time-harmonic maxwell equations. Math. Comp., 72(243):1179-1214, 2003.

129. A. Quarteroni. Numerical models for differential problems, volume 2. Springer Science \& Business Media, 2014.

130. P.-A. Raviart and J.-M. Thomas. Introduction à l' analyse numérique des équations aux dérivées partielles. Masson, 1983.

131. B. Rivière, S. Shaw, M. F. Wheeler, and J. R. Whiteman. Discontinuous Galerkin finite element methods for linear elasticity and quasistatic linear viscoelasticity. Numer. Math., 95(2):347-376, 2003.

132. B. Rivière and M. F. Wheeler. Discontinuous finite element methods for acoustic and elastic wave problems. Contemporary Mathematics, 329:271-282, 2003.

133. N. Schwenck, B. Flemisch, R. Helmig, and B. I. Wohlmuth. Dimensionally reduced flow models in fractured porous media: crossings and boundaries. Comput. Geosci., 19(6):1219-1230, 2015.

134. C. P. Simon and L. E. Blume. Mathematics for Economists. W. W. Norton and Company: New York, 1996.

135. A. Sommariva and M. Vianello. Product Gauss cubature over polygons based on Green's integration formula. BIT, $47(2): 441$ - 453, 2007.

136. E. Stein. Singular Integrals and Differentiability Properties of Functions. Princeton University Press, Princeton, N.J, 1970.

137. A. H. Stroud and D. Secrest. Gaussiam quadrature formulas. ZAMM Z. Angew. Math. Mech., 47(2):138 - 139, 1967.

138. Y. Sudhakar and W. A. Wall. Quadrature schemes for arbitrary convex/concave volumes and integration of weak form in enriched partition of unity methods. Comput. Methods Appl. Mech. Engrg., 258(1):39 - 54, 2013.

139. N. Sukumar, N. Moes, and T. Belytschko. Extended finite element method for three-dimensional crack modelling. Internat. J. Numer. Methods Engrg., 48(11):1549 - 1570, August 2000.

140. N. Sukumar and A. Tabarraei. Conforming Polygonal Finite Elements. Internat. J. Numer. Methods Engrg., 61(12):2045-2066, 2004. 
141. A. Tabarraei and N. Sukumar. Extended Finite Element Method on polygonal and quadtree meshes. Comput. Methods Appl. Mech. Engrg., 197(5):425-438, 2008.

142. C. Talischi, G. H. Paulino, A. Pereira, and I. F. Menezes. Polymesher: a general-purpose mesh generator for polygonal elements written in matlab. Structural and Multidisciplinary Optimization, 45(3):309-328, 2012.

143. M. E. Taylor. Partial Differential Equations: Basic Theory. Springer-Verlag, New york, 1996.

144. S. Terrana, J. P. Vilotte, and L. Guillot. A spectral hybridizable discontinuous galerkin method for elastic-acoustic wave propagation. Geophys. J. Int., 213:574-602, 2018.

145. G. Ventura. On the elimination of quadrature subcells for discontinuous functions in the extended finite-element method. Internat. $J$. Numer. Methods Engrg., 66(5):761 - 795, 2006.

146. G. Ventura and E. Benvenuti. Equivalent polynomials for quadrature in Heaviside function enriched elements. Internat. J. Numer. Methods Engrg., 102(3 - 4):688 - 710, 2015.

147. T. Warburton and J. S. Hesthaven. On the constants in $h p$-finite element trace inverse inequalities. Comput. Methods Appl. Mech. Engrg., 192(25):2765-2773, 2003.

148. M. F. Wheeler. An elliptic collocation-finite element method with interior penalties. SIAM J. Numer. Anal., 15(1):152-161, 1978.

149. L. C. Wilcox, G. Stadler, C. Burstedde, and O. Ghattas. A high-order discontinuous galerkin method for wave propagation through coupled elastic-acoustic media. J. Comput. Phys., 229:9373-9396, 2010.

150. N. Yarvin and V. Rokhlin. Generalized Gaussian quadratures and singular value decompositions of integral operators. SIAM J. Sci. Comput., 20(2):669 - 718, 1998.

151. A. M. Yogitha and K. T. Shivaram. Numerical integration of arbitrary functions over a convex and non convex polygonal domain by eight noded linear quadrilateral finite element method. Aust. J. Basic Appl. Sci., 10(16):104 - 110, 2016. 